\title{
Description of Incomplete Financial Markets for Time Evolution of Risk Assets
}

\author{
Nicholas Simon Gonchar \\ Bogolyubov Institute for Theoretical Physics of NAS, Kiev, Ukraine \\ Email:mhonchar@i.ua
}

How to cite this paper: Gonchar, N.S. (2019) Description of Incomplete Financial Markets for Time Evolution of Risk Assets. Advances in Pure Mathematics, 9, 567-610. https://doi.org/10.4236/apm.2019.96029

Received: June 4, 2019

Accepted: June 27, 2019

Published: June 30, 2019

Copyright $\odot 2019$ by author(s) and Scientific Research Publishing Inc. This work is licensed under the Creative Commons Attribution International License (CC BY 4.0).

http://creativecommons.org/licenses/by/4.0/

\begin{abstract}
In the paper, a class of discrete evolutions of risk assets having the memory is considered. For such evolutions the description of all martingale measures is presented. It is proved that every martingale measure is an integral on the set of extreme points relative to some measure on it. For such a set of evolutions of risk assets, the contraction of the set of martingale measures on the filtration is described and the representation for it is found. The inequality for the integrals from a nonnegative random value relative to the contraction of the set of martingale measure on the filtration which is dominated by one is obtained. Using these inequalities a new proof of the optional decomposition theorem for super-martingales is presented. The description of all local regular super-martingales relative to the regular set of measures is presented. The applications of the results obtained to mathematical finance are presented. In the case, as evolution of a risk asset is given by the discrete geometric Brownian motion, the financial market is incomplete and a new formula for the fair price of super-hedge is founded.
\end{abstract}

\section{Keywords}

Random Process, Regular Set of Measures, Optional Doob Decomposition, Local Regular Super-Martingale, Martingale, Discrete Geometric Brownian Motion

\section{Introduction}

In the paper, the notion of the regular super-martingale relative to the set of equivalent measures is introduced. The necessary and sufficient conditions of the regular super-martingale relative to the set of equivalent measures are found. The notion of the family of equivalent measures consistent with filtration is introduced. Theorem giving the sufficient conditions of the existence of su- 
per-martingale and martingale relative to the set of equivalent measures consistent with the filtration is proved. The sufficient conditions of the existence of the set of equivalent measures consistent with the filtration, satisfying the conditions: the mean value of the nonnegative random value relative to these set of measures equal one, are given. Further, we construct the set of equivalent measures consistent with the filtration satisfying the above conditions. First, we give the complete description of the set of equivalent measures satisfying the conditions: the mean value of the nonnegative random value relative to this set of measures equals one. Using the above result we construct the example of the set of equivalent measures consistent with the filtration satisfying the condition: the mean value of the nonnegative random value relative to every measure of this set of measures equals one. The above method we use for the construction of evolution of risk assets and we describe completely the set of equivalent martingale measures for this evolution. We prove that every martingale measure is an integral on the set of extreme points of the convex set of martingale measures relative to some measure on it. To give a new proof of the optional decomposition for super-martingale we describe the contraction of every martingale measure on the filtration and find the closure of integrals from the integrable random values over all martingale measures. To do this we introduce the notion of the exhaustive decomposition and prove that every separable metric space with the Borel $\sigma$-algebra has an exhaustive decomposition. For the integral from the nonnegative random value relative to all martingale measures which is dominated by one, the inequalities for this random value are obtained. This fact gives us the possibility to find a new proof of the optional decomposition for the nonnegative super-martingale. This proof does not use the no-arbitrage arguments and the measurable choice [1] [2] [3] [4]. This paper is a generalization of the results of the paper [5].

First, the optional decomposition for diffusion processes super-martingale was opened by El Karoui N. and Quenez M. C. [6]. After that, Kramkov D. O. and Follmer H. [1] [2] proved the optional decomposition for the nonnegative bounded super-martingales. Folmer H. and Kabanov Yu. M. [3] [4] proved analogous result for an arbitrary super-martingale. Recently, Bouchard B. and Nutz M. [7] considered a class of discrete models and proved the necessary and sufficient conditions for the validity of the optional decomposition.

The optional decomposition for super-martingales plays the fundamental role for the risk assessment in incomplete markets [1] [2] [6] [8] [9] [10] [11].

At last, we consider an application of the results obtained to find the new formula for the fair price of super-hedge in the case, as the risk asset evolves by the discrete geometric Brownian motion.

\section{Local Regular Super-Martingales Relative to a Set of Equivalent Measures}

We assume that on a measurable space $\{\Omega, \mathcal{F}\}$ a filtration $\mathcal{F}_{m} \subset \mathcal{F}_{m+1} \subset \mathcal{F}$, 
$m=\overline{0, \infty}$, and a set of equivalent measures $M$ on $\mathcal{F}$ are given. Further, we assume that $\mathcal{F}_{0}=\{\varnothing, \Omega\}$ and the $\sigma$-algebra $\mathcal{F}=\sigma\left(\bigvee_{n=1}^{\infty} \mathcal{F}_{n}\right)$ is a minimal $\sigma$-algebra generated by the algebra $\bigvee_{n=1}^{\infty} \mathcal{F}_{n}$. A random process $\psi=\left\{\psi_{m}\right\}_{m=0}^{\infty}$ is said to be adapted one relative to the filtration $\left\{\mathcal{F}_{m}\right\}_{m=0}^{\infty}$, if $\psi_{m}$ is a $\mathcal{F}_{m}$ measurable random value, $m=\overline{0, \infty}$.

Definition 1. An adapted random process $f=\left\{f_{m}\right\}_{m=0}^{\infty}$ is said to be a super-martingale relative to the filtration $\mathcal{F}_{m}, m=\overline{0, \infty}$, and the family of equivalent measures $M$, if $E^{P}\left|f_{m}\right|<\infty, m=\overline{1, \infty}, P \in M$, and the inequalities

$$
E^{P}\left\{f_{m} \mid \mathcal{F}_{k}\right\} \leq f_{k}, 0 \leq k \leq m, m=\overline{1, \infty}, P \in M,
$$

are valid.

Further, for an adapted process $f$ we use both the denotation $\left\{f_{m}, \mathcal{F}_{m}\right\}_{m=0}^{\infty}$ and the denotation $\left\{f_{m}\right\}_{m=0}^{\infty}$.

Definition 2. A super-martingale $\left\{f_{m}, \mathcal{F}_{m}\right\}_{m=0}^{\infty}$ relative to a set of equivalent measures $M$ is a local regular one, if $\sup _{P \in M} E^{P}\left|f_{m}\right|<\infty, m=\overline{1, \infty}$, and there exists an adapted nonnegative increasing random process $\left\{g_{m}, \mathcal{F}_{m}\right\}_{m=0}^{\infty}, g_{0}=0$, $\sup _{P \in M} E^{P}\left|g_{m}\right|<\infty, m=\overline{1, \infty}$, such that $\left\{f_{m}+g_{m}, \mathcal{F}_{m}\right\}_{m=0}^{\infty}$ is a martingale relative to every measure from $M$.

The next elementary Theorem 1 will be very useful later.

Theorem 1. Let a super-martingale $\left\{f_{m}, \mathcal{F}_{m}\right\}_{m=0}^{\infty}$, relative to a set of equivalent measures $M$ be such that $\sup _{P \in M} E^{P}\left|f_{m}\right|<\infty, m=\overline{1, \infty}$. The necessary and sufficient condition for it to be a local regular one is the existence of an adapted nonnegative random process $\left\{\bar{g}_{m}^{0}, \mathcal{F}_{m}\right\}_{m=0}^{\infty}, \sup _{P \in M} E^{P}\left|\bar{g}_{m}^{0}\right|<\infty, m=\overline{1, \infty}$, such that

$$
f_{m-1}-E^{P}\left\{f_{m} \mid \mathcal{F}_{m-1}\right\}=E^{P}\left\{\bar{g}_{m}^{0} \mid \mathcal{F}_{m-1}\right\}, m=\overline{1, \infty}, \quad P \in M .
$$

Proof. The necessity. If $\left\{f_{m}, \mathcal{F}_{m}\right\}_{m=0}^{\infty}$ is a local regular super-martingale, then there exist a martingale $\left\{\bar{M}_{m}, \mathcal{F}_{m}\right\}_{m=0}^{\infty}$ and a non-decreasing nonnegative random process $\left\{g_{m}, \mathcal{F}_{m}\right\}_{m=0}^{\infty}, g_{0}=0$, such that

$$
f_{m}=\bar{M}_{m}-g_{m}, m=\overline{1, \infty} .
$$

From here, we obtain the equalities

$$
\begin{aligned}
& E^{P}\left\{f_{m-1}-f_{m} \mid \mathcal{F}_{m-1}\right\} \\
& =E^{P}\left\{g_{m}-g_{m-1} \mid \mathcal{F}_{m-1}\right\}=E^{P}\left\{\bar{g}_{m}^{0} \mid \mathcal{F}_{m-1}\right\}, m=\overline{1, \infty}, P \in M,
\end{aligned}
$$

where we introduced the denotation $\bar{g}_{m}^{0}=g_{m}-g_{m-1} \geq 0$. It is evident that $E^{P} \bar{g}_{m}^{0} \leq \sup _{P \in M} E^{P} g_{m}+\sup _{P \in M} E^{P} g_{m-1}<\infty$.

The sufficiency. Suppose that there exists an adapted nonnegative random process $\bar{g}^{0}=\left\{\bar{g}_{m}^{0}\right\}_{m=0}^{\infty}, \bar{g}_{0}^{0}=0, E^{P} \bar{g}_{m}^{0}<\infty, m=\overline{1, \infty}$, such that the equalities (2) 
hold. Let us consider the random process $\left\{\bar{M}_{m}, \mathcal{F}_{m}\right\}_{m=0}^{\infty}$, where

$$
\bar{M}_{0}=f_{0}, \quad \bar{M}_{m}=f_{m}+\sum_{i=1}^{m} \bar{g}_{m}^{0}, \quad m=\overline{1, \infty} .
$$

It is evident that $E^{P}\left|\bar{M}_{m}\right|<\infty$ and

$$
E^{P}\left\{\bar{M}_{m-1}-\bar{M}_{m} \mid \mathcal{F}_{m-1}\right\}=E^{P}\left\{f_{m-1}-f_{m}-\bar{g}_{m}^{0} \mid \mathcal{F}_{m-1}\right\}=0 .
$$

Theorem 1 is proved.

Lemma 1. Any super-martingale $\left\{f_{m}, \mathcal{F}_{m}\right\}_{m=0}^{\infty}$ relative to a family of measures $M$ for which there hold equalities $E^{P} f_{m}=f_{0}, m=\overline{1, \infty}, P \in M$, is a martingale with respect to this family of measures and the filtration $\mathcal{F}_{m}, m=\overline{1, \infty}$.

Proof. The proof of Lemma 1 see [12].

In the next Lemma, we present the formula for calculation of the conditional expectation relative to another measure from $M$.

Lemma 2. On the measurable space $\{\Omega, \mathcal{F}\}$ with the filtration $\mathcal{F}_{n}$ on it, let $M$ be a set of equivalent measures and let $\xi$ be an integrable random value. Then, the following formulas

$$
E^{P_{1}}\left\{\xi \mid \mathcal{F}_{n}\right\}=E^{P_{2}}\left\{\xi \varphi_{n}^{P_{1}} \mid F_{n}\right\}, n=\overline{1, \infty}
$$

are valid, where

$$
\varphi_{n}^{P_{1}}=\frac{\mathrm{d} P_{1}}{\mathrm{~d} P_{2}}\left[E^{P_{2}}\left\{\frac{\mathrm{d} P_{1}}{\mathrm{~d} P_{2}} \mid \mathcal{F}_{n}\right\}\right]^{-1}, P_{1}, P_{2} \in M .
$$

Proof. The proof of Lemma 2 is evident.

\section{Local Regular Super-Martingales Relative to a Set of Equivalent Measures Consistent with the Filtration}

Definition 3. On a measurable space $\{\Omega, \mathcal{F}\}$ with a filtration $\mathcal{F}_{n}$ on it, a set of equivalent measures $M$ we call consistent with the filtration $\mathcal{F}_{n}$, if for every pair of measures $\left(Q_{1}, Q_{2}\right) \in M^{2}$ the set of measures

$$
R_{s}^{k}(A)=\int_{A} \frac{E^{Q_{1}}\left\{\frac{\mathrm{d} Q_{2}}{\mathrm{~d} Q_{1}} \mid \mathcal{F}_{k}\right\}}{E^{Q_{1}}\left\{\frac{\mathrm{d} Q_{2}}{\mathrm{~d} Q_{1}} \mid \mathcal{F}_{s}\right\}} \mathrm{d} Q_{1}, A \in \mathcal{F}, k \geq s \geq n, n=\overline{0, \infty},
$$

belongs to the set $M$, where $M^{2}$ is a direct product of the set $M$ by itself.

Lemma 3. On the measurable space $\{\Omega, \mathcal{F}\}$ with the filtration $\mathcal{F}_{n}$ on it, the set of measures

$$
M=\left\{Q, Q(A)=\int_{A} \alpha(\omega) \mathrm{d} P, A \in \mathcal{F}, Q(\Omega)=1\right\}
$$

is a consistent one with the filtration $\mathcal{F}_{n}$, if $P$ is a measure on $\{\Omega, \mathcal{F}\}$ and a random value $\alpha(\omega)$ runs over all nonnegative random values, satisfying the condition $P(\{\omega, \alpha(\omega)>0\})=1$.

Proof. Suppose that $\left(Q_{1}, Q_{2}\right)$ belongs to $M^{2}$. Then, $\frac{\mathrm{d} Q_{2}}{\mathrm{~d} Q_{1}}=\frac{\alpha_{2}(\omega)}{\alpha_{1}(\omega)}$ and 
$P\left(\left\{\omega, \frac{\mathrm{d} Q_{2}}{\mathrm{~d} Q_{1}}>0\right\}\right)=1$, since the equalities $P\left(\left\{\omega, 0<\alpha_{1}(\omega)<\infty\right\}\right)=1$, $P\left(\left\{\omega, 0<\alpha_{2}(\omega)<\infty\right\}\right)=1$ are true. It is evident that

$$
\begin{aligned}
R_{s}^{k}(A) & =\int_{A} \frac{E^{Q_{1}}\left\{\frac{\mathrm{d} Q_{2}}{\mathrm{~d} Q_{1}} \mid \mathcal{F}_{k}\right\}}{Q^{Q_{1}}\left\{\frac{\mathrm{d} Q_{2}}{\mathrm{~d} Q_{1}} \mid \mathcal{F}_{s}\right\}} \mathrm{d} Q_{1} \\
& =\int_{A} \frac{E^{Q_{1}}\left\{\frac{\mathrm{d} Q_{2}}{\mathrm{~d} Q_{1}} \mid \mathcal{F}_{k}\right\}}{E^{Q_{1}}\left\{\frac{\mathrm{d} Q_{2}}{\mathrm{~d} Q_{1}} \mid \mathcal{F}_{s}\right\}} \alpha_{1}(\omega) \mathrm{d} P, A \in \mathcal{F}, k \geq s \geq n, n=\overline{0, \infty} .
\end{aligned}
$$

It is easy to see that

$$
P\left(\left\{\omega, \frac{E^{Q_{1}}\left\{\frac{\mathrm{d} Q_{2}}{\mathrm{~d} Q_{1}} \mid \mathcal{F}_{k}\right\}}{E^{Q_{1}}\left\{\frac{\mathrm{d} Q_{2}}{\mathrm{~d} Q_{1}} \mid \mathcal{F}_{s}\right\}} \alpha_{1}(\omega)>0\right\}\right)=1, k \geq s,
$$

since

$$
\begin{gathered}
P\left(\left\{\omega, E^{Q_{1}}\left\{\frac{\mathrm{d} Q_{2}}{\mathrm{~d} Q_{1}} \mid \mathcal{F}_{k}\right\}>0\right\}\right)=1, k \geq s, \\
P\left(\left\{\omega, 0<E^{Q_{1}}\left\{\frac{\mathrm{d} Q_{2}}{\mathrm{~d} Q_{1}} \mid \mathcal{F}_{s}\right\}<\infty\right\}\right)=1, s \geq n, n=\overline{0, \infty} .
\end{gathered}
$$

The last equality follows from the equivalence of the measures $Q_{1}, Q_{2}$ and $P$. Altogether, it means that the set of measures $R_{s}^{k}, k \geq s \geq n, n=\overline{0, \infty}$, belongs to the set $M$. The same is true for the pair $\left(Q_{2}, Q_{1}\right) \in M^{2}$. Lemma 3 is proved.

Theorem 2. On the measurable space $\{\Omega, \mathcal{F}\}$ with the filtration $\mathcal{F}_{n}$ on it, let the set of equivalent measures $M$ be consistent with the filtration $\mathcal{F}_{n}$. Then, for every nonnegative random value $\xi$ such that $\sup _{P \in M} E^{P} \xi<\infty$, the random process $\left\{f_{n}, \mathcal{F}_{n}\right\}_{n=0}^{\infty}$ is a super-martingale relative to the set of measures $M$, where $f_{n}=\operatorname{ess} \sup _{P \in M} E^{P}\left\{\xi \mid \mathcal{F}_{n}\right\}, n=\overline{0, \infty}$.

Proof. Let $Q \in M$, then, due to Lemma 2, for every $P \in M$

$$
E^{P}\left\{\xi \mid \mathcal{F}_{n}\right\}=E^{Q}\left\{\xi\left|\frac{\frac{\mathrm{d} P}{\mathrm{~d} Q}}{E^{Q}\left\{\frac{\mathrm{d} P}{\mathrm{~d} Q} \mid \mathcal{F}_{n}\right\}}\right| \mathcal{F}_{n}\right\} .
$$

If to put instead of the measure $P$ the measure $R_{s}^{k}, k \geq s \geq n$, for the pair of measures $(Q, P)$ we obtain 


$$
\begin{aligned}
& E^{R_{s}^{k}}\left\{\xi \mid F_{n}\right\}=E^{Q}\left\{\xi\left|\frac{\frac{\mathrm{d} R_{s}^{k}}{\mathrm{~d} Q}}{E^{Q}\left\{\frac{\mathrm{d} R_{s}^{k}}{\mathrm{~d} Q} \mid \mathcal{F}_{n}\right\}}\right| \mathcal{F}_{n}\right\} \\
& =E^{Q}\left\{\frac{E^{Q}\left\{\frac{\mathrm{d} P}{\mathrm{~d} Q} \mid \mathcal{F}_{k}\right\}}{E^{Q}\left\{\frac{\mathrm{d} P}{\mathrm{~d} Q} \mid \mathcal{F}_{s}\right\}} \mid \mathcal{F}_{n}\right\},
\end{aligned}
$$

where we took into account the equality

$$
E^{Q}\left\{\frac{\mathrm{d} R_{s}^{k}}{\mathrm{~d} Q} \mid \mathcal{F}_{n}\right\}=E^{Q}\left\{\frac{E^{Q}\left\{\frac{\mathrm{d} P}{\mathrm{~d} Q} \mid \mathcal{F}_{k}\right\}}{E^{Q}\left\{\frac{\mathrm{d} P}{\mathrm{~d} Q} \mid \mathcal{F}_{s}\right\}} \mid \mathcal{F}_{n}\right\}=1, k \geq s \geq n .
$$

From the formula (16), it follows the equality

$$
\text { ess } \sup _{P \in M} E^{P}\left\{\xi \mid \mathcal{F}_{n}\right\}=\operatorname{ess} \sup _{T \in R_{n}} E^{P}\left\{\xi T \mid \mathcal{F}_{n}\right\}
$$

where $R_{n}$ is a set of martingales $T=\left\{T_{m}\right\}_{m=0}^{\infty}$ relative to the measure $Q$ such that $T_{m}=1, m \leq n, T_{m}=\frac{E^{Q}\left\{\frac{\mathrm{d} P}{\mathrm{~d} Q} \mid \mathcal{F}_{m}\right\}}{E^{Q}\left\{\frac{\mathrm{d} P}{\mathrm{~d} Q} \mid \mathcal{F}_{s}\right\}}, m \geq s \geq n, P \in M$. The definition of ess sup for the uncountable set of random values see [13]. It is evident that $T_{n} \subseteq T_{n-1}$. Let us consider

$$
\begin{aligned}
& E^{Q}\left\{\operatorname{esssup}_{P \in M} E^{P}\left\{\xi \mid \mathcal{F}_{n}\right\} \mid \mathcal{F}_{n-1}\right\}=E^{Q}\left\{\operatorname{ess}_{T \in R_{n}} E^{P}\left\{\xi T \mid \mathcal{F}_{n}\right\} \mid \mathcal{F}_{n-1}\right\} \\
& =E^{Q}\left\{\sup _{i \geq 1} E^{P}\left\{\xi T_{i} \mid \mathcal{F}_{n}\right\} \mid \mathcal{F}_{n-1}\right\}=E^{Q}\left\{\lim _{k \rightarrow \infty} \max _{1 \leq i \leq k} E^{P}\left\{\xi T_{i} \mid \mathcal{F}_{n}\right\} \mid \mathcal{F}_{n-1}\right\} \\
& =\lim _{k \rightarrow \infty} E^{Q}\left\{\max _{1 \leq i \leq k} E^{P}\left\{\xi T_{i} \mid \mathcal{F}_{n}\right\} \mid \mathcal{F}_{n-1}\right\}=\lim _{k \rightarrow \infty} E^{P}\left\{\xi T_{\tau_{k}} \mid \mathcal{F}_{n-1}\right\} \\
& \leq \operatorname{ess} \sup _{T \in R_{n}} E^{Q}\left\{\xi T \mid \mathcal{F}_{n-1}\right\} \leq \text { ess } \sup _{T \in R_{n-1}} E^{Q}\left\{\xi T \mid \mathcal{F}_{n-1}\right\} \\
& =\operatorname{ess} \sup _{P \in M} E^{Q}\left\{\xi \mid \mathcal{F}_{n-1}\right\},
\end{aligned}
$$

where

$$
\begin{aligned}
& \tau_{1}=1, \\
& \tau_{i}=\left\{\begin{array}{ll}
\tau_{i-1}, & E^{P}\left\{\xi T_{\tau_{i-1}} \mid \mathcal{F}_{n}\right\}>E^{P}\left\{\xi T_{i} \mid \mathcal{F}_{n}\right\}, \\
i, & E^{P}\left\{\xi T_{\tau_{i-1}} \mid \mathcal{F}_{n}\right\} \leq E^{P}\left\{\xi T_{i} \mid \mathcal{F}_{n}\right\},
\end{array} \quad i=\overline{2, k} .\right.
\end{aligned}
$$

Lemma 2 is proved.

Theorem 3. On the measurable space $\{\Omega, \mathcal{F}\}, \mathcal{F}=\sigma\left(\bigvee_{i=1}^{\infty} \mathcal{F}_{i}\right)$, let $M$ be a set of equivalent measures being consistent with the filtration $\mathcal{F}_{n}$. If there exists a nonnegative random value $\xi \neq 1$ such that $E^{P} \xi=1, P \in M$, then 
$E^{P}\left\{\xi \mid \mathcal{F}_{n}\right\}, P \in M$, is a local regular martingale.

Proof. Due to Lemma 2, the random process $\left\{f_{n}, \mathcal{F}_{n}\right\}_{n=0}^{\infty}$, where $f_{n}=\operatorname{ess} \sup _{P \in M} E^{P}\left\{\xi \mid \mathcal{F}_{n}\right\}, n=\overline{0, \infty}$, is a super-martingale relative to the set of measures $M$, that is,

$$
E^{Q}\left\{\operatorname{ess} \sup _{P \in M} E^{P}\left\{\xi \mid \mathcal{F}_{n}\right\} \mid \mathcal{F}_{n-1}\right\} \leq \operatorname{ess} \sup _{P \in M} E^{P}\left\{\xi \mid \mathcal{F}_{n-1}\right\}, Q \in M, n=\overline{0, \infty} .
$$

From the inequality (22), it follows the inequality

$$
E^{Q} \operatorname{ess} \sup _{P \in M} E^{P}\left\{\xi \mid \mathcal{F}_{n}\right\} \leq 1, n=\overline{0, \infty} .
$$

Since $E^{Q} \operatorname{ess} \sup E^{P}\left\{\xi \mid \mathcal{F}_{n}\right\} \geq E^{Q} E^{Q}\left\{\xi \mid \mathcal{F}_{n}\right\}=1$, we have

$$
E^{Q} \operatorname{ess} \sup _{P \in M} E^{P}\left\{\xi \mid \mathcal{F}_{n}\right\}=1, Q \in M, n=\overline{0, \infty} .
$$

The inequalities (22) and the equalities (24) give the equalities

$$
E^{Q}\left\{\operatorname{esssup}_{P \in M} E^{P}\left\{\xi \mid \mathcal{F}_{n}\right\} \mid \mathcal{F}_{n-1}\right\}=\operatorname{ess} \sup _{P \in M} E^{P}\left\{\xi \mid \mathcal{F}_{n-1}\right\}, Q \in M, n=\overline{1, \infty},
$$

which are true with the probability 1 . The last means that $\left\{f_{n}, \mathcal{F}_{n}\right\}_{n=0}^{\infty}$ is a martingale relative to the set of measures $M$, where $f_{n}=\operatorname{ess} \sup _{P \in M} E^{P}\left\{\xi \mid \mathcal{F}_{n}\right\}, n=\overline{0, \infty}$. With the probability $1, \lim _{n \rightarrow \infty} \operatorname{ess} \sup _{P \in M} E^{P}\left\{\xi \mid \mathcal{F}_{n}\right\}=f_{\infty}$, where the random value $f_{\infty}$ is $F$ measurable one. From the inequality (23) and Fatou Lemma [13] [14], we obtain

$$
E^{P} f_{\infty} \leq 1, P \in M .
$$

Prove that $f_{\infty}=\xi$. Going to the limit in the inequality

$$
\operatorname{ess} \sup _{P \in M} E^{P}\left\{\xi \mid \mathcal{F}_{n}\right\} \geq E^{P_{1}}\left\{\xi \mid \mathcal{F}_{n}\right\},
$$

as $n \rightarrow \infty$, we obtain the inequality

$$
f_{\infty} \geq \xi \text {. }
$$

From the inequality (26) and the inequality (28), we obtain the inequalities $1 \geq E^{P} f_{\infty} \geq E^{P} \xi=1$. Or, $E^{P} f_{\infty}=1$. The equalities $E^{P} f_{\infty}=1, E^{P} \xi=1$ and the inequality (28) give the equality $f_{\infty}=\xi$ with the probability 1 . Lemma 3 is proved.

Lemma 4. On the measurable space $\{\Omega, \mathcal{F}\}$ with the filtration $\mathcal{F}_{n}$ on it, let there exist $k$ equivalent measures $P_{1}, \cdots, P_{k}, k>1$, and a nonnegative random value $\xi_{0} \neq 1$ be such that

$$
E^{P_{i}}\left\{\xi_{0} \mid \mathcal{F}_{n}\right\}=E^{P_{1}}\left\{\xi_{0} \mid \mathcal{F}_{n}\right\}, E^{P_{i}} \xi_{0}=1, i=\overline{2, k}, n=\overline{0, \infty} .
$$

Then, there exists the set of equivalent measures $M$ consistent with the filtration $\mathcal{F}_{n}$, satisfying the condition $E^{P} \xi_{0}=1, P \in M$.

Proof. Let us consider the set of equivalent measures $M$, satisfying the condition

$$
E^{P}\left\{\xi_{0} \mid \mathcal{F}_{n}\right\}=E^{P_{1}}\left\{\xi_{0} \mid \mathcal{F}_{n}\right\}, n=\overline{0, \infty}, P \in M .
$$


Such a set of measures is a nonempty one. Suppose that $Q_{1}, Q_{2} \in M$, then

$$
E^{Q_{1}}\left\{\xi_{0} \mid \mathcal{F}_{n}\right\}=E^{Q_{2}}\left\{\xi_{0} \mid \mathcal{F}_{n}\right\}, n=\overline{0, \infty} .
$$

Let us prove that the formula

$$
E^{Q_{1}}\left\{\xi_{0} \frac{E^{Q_{1}}\left\{\frac{\mathrm{d} Q_{2}}{\mathrm{~d} Q_{1}} \mid \mathcal{F}_{k}\right\}}{E^{Q_{1}}\left\{\frac{\mathrm{d} Q_{2}}{\mathrm{~d} Q_{1}} \mid \mathcal{F}_{s}\right\}} \mid \mathcal{F}_{n}\right\}=E^{Q_{1}}\left\{\xi_{0} \mid \mathcal{F}_{n}\right\}, n \leq s \leq k, n=\overline{0, \infty},
$$

is valid. Let $s \geq n$. Then, from the equalities (31), we have

$$
E^{Q_{1}}\left\{E^{Q_{2}}\left\{\xi_{0} \mid \mathcal{F}_{s}\right\} \mid \mathcal{F}_{n}\right\}=E^{Q_{1}}\left\{\xi_{0} \mid \mathcal{F}_{n}\right\} .
$$

Let $k \geq s$. Then,

$$
\begin{aligned}
& E^{Q_{1}}\left\{E^{Q_{2}}\left\{\xi_{0} \mid \mathcal{F}_{s}\right\} \mid \mathcal{F}_{n}\right\} \\
& =E^{Q_{1}}\left\{E^{Q_{2}}\left\{E^{Q_{2}}\left\{\xi_{0} \mid \mathcal{F}_{k}\right\} \mid \mathcal{F}_{s}\right\} \mid \mathcal{F}_{n}\right\} \\
& =E^{Q_{1}}\left\{E^{Q_{1}}\left\{E^{Q_{2}}\left\{\xi_{0} \mid \mathcal{F}_{k}\right\} \frac{\frac{\mathrm{d} Q_{2}}{\mathrm{~d} Q_{1}}}{E^{Q_{1}}\left\{\frac{\mathrm{d} Q_{2}}{\mathrm{~d} Q_{1}} \mid \mathcal{F}_{s}\right\}} \mid \mathcal{F}_{s}\right\} \mid \mathcal{F}_{n}\right\} \\
& =E^{Q_{1}}\left\{E^{Q_{2}}\left\{\xi_{0} \mid \mathcal{F}_{k}\right\} \frac{\frac{\mathrm{d} Q_{2}}{\mathrm{~d} Q_{1}}}{E^{Q_{1}}\left\{\frac{\mathrm{d} Q_{2}}{\mathrm{~d} Q_{1}} \mid \mathcal{F}_{s}\right\}} \mid \mathcal{F}_{n}\right\} \\
& =E^{Q_{1}}\left\{E^{Q_{1}}\left\{\xi_{0} \mid \mathcal{F}_{k}\right\} \frac{\frac{\mathrm{d} Q_{2}}{\mathrm{~d} Q_{1}}}{E^{Q_{1}}\left\{\frac{\mathrm{d} Q_{2}}{\mathrm{~d} Q_{1}} \mid \mathcal{F}_{s}\right\}} \mid \mathcal{F}_{n}\right\} \\
& =E^{Q_{1}}\left\{E^{Q_{1}}\left\{\xi_{0} \mid \mathcal{F}_{k}\right\} \frac{E^{Q_{1}}\left\{\frac{\mathrm{d} Q_{2}}{\mathrm{~d} Q_{1}} \mid \mathcal{F}_{k}\right\}}{E^{Q_{1}}\left\{\frac{\mathrm{d} Q_{2}}{\mathrm{~d} Q_{1}} \mid \mathcal{F}_{s}\right\}} \mid \mathcal{F}_{n}\right\} \\
& =E^{Q_{1}}\left\{\xi_{0}\left|\frac{E^{Q_{1}}\left\{\frac{\mathrm{d} Q_{2}}{\mathrm{~d} Q_{1}} \mid \mathcal{F}_{k}\right\}}{E^{Q_{1}}\left\{\frac{\mathrm{d} Q_{2}}{\mathrm{~d} Q_{1}} \mid \mathcal{F}_{s}\right\}}\right| \mathcal{F}_{n}\right\} .
\end{aligned}
$$

This proves the formula (32). To finish the proof of Lemma 4, it needs to prove that the set of measures

$$
R_{s}^{k}(A)=\int_{A} \frac{E^{Q_{1}}\left\{\frac{\mathrm{d} Q_{2}}{\mathrm{~d} Q_{1}} \mid \mathcal{F}_{k}\right\}}{E^{Q_{1}}\left\{\frac{\mathrm{d} Q_{2}}{\mathrm{~d} Q_{1}} \mid \mathcal{F}_{s}\right\}} \mathrm{d} Q_{1}, \quad A \in \mathcal{F}, k \geq s \geq n, \quad n=\overline{0, \infty},
$$

belongs to the set $M$. Really, 


$$
\begin{aligned}
& E^{R_{s}^{k}}\left\{\xi_{0} \mid \mathcal{F}_{n}\right\}=E^{Q_{1}}\left\{\xi_{0}\left|\frac{\frac{\mathrm{d} R_{s}^{k}}{\mathrm{~d} Q_{1}}}{E^{Q_{1}}\left\{\frac{\mathrm{d} R_{s}^{k}}{\mathrm{~d} Q_{1}} \mid \mathcal{F}_{n}\right\}}\right| \mathcal{F}_{n}\right\} \\
& =E^{Q_{1}}\left\{\xi_{0} \frac{E^{Q_{1}}\left\{\frac{\mathrm{d} Q_{2}}{\mathrm{~d} Q_{1}} \mid \mathcal{F}_{k}\right\}}{E^{Q_{1}}\left\{\frac{\mathrm{d} Q_{2}}{\mathrm{~d} Q_{1}} \mid \mathcal{F}_{s}\right\}} \mid \mathcal{F}_{n}\right\} \\
& =E^{Q_{1}}\left\{\xi_{0} \mid \mathcal{F}_{n}\right\},
\end{aligned}
$$

where we took into account the equality

$$
E^{Q_{1}}\left\{\frac{\mathrm{d} R_{s}^{k}}{\mathrm{~d} Q_{1}} \mid \mathcal{F}_{n}\right\}=E^{Q_{1}}\left\{\frac{E^{Q_{1}}\left\{\frac{\mathrm{d} Q_{2}}{\mathrm{~d} Q_{1}} \mid \mathcal{F}_{k}\right\}}{E^{Q_{1}}\left\{\frac{\mathrm{d} Q_{2}}{\mathrm{~d} Q_{1}} \mid \mathcal{F}_{s}\right\}} \mid \mathcal{F}_{n}\right\}=1, k \geq s \geq n .
$$

From this, it follows that the set of measures $R_{s}^{k} \in M$. This proves the consistence with the filtration of the set of measures $M$. Lemma 4 is proved.

The next Lemma 5 is a key statement in the construction of the set of measures satisfying the conditions of Lemma 4.

On a probability space $\{\Omega, \mathcal{F}, P\}$, let $\xi$ be a random value, satisfying the conditions

$$
0<P(\{\omega, \xi>0\})<1,0<P(\{\omega, \xi<0\}) .
$$

Denote $\Omega^{+}=\{\omega, \xi(\omega)>0\}, \Omega^{-}=\{\omega, \xi(\omega) \leq 0\}$ and let $\mathcal{F}^{-}, \mathcal{F}^{+}$be the restrictions of the $\sigma$-algebra $\mathcal{F}$ on the sets $\Omega^{-}$and $\Omega^{+}$, correspondingly. Suppose that $P^{-}$and $P^{+}$are the contractions of the measure $P$ on the $\sigma$-algebras $\mathcal{F}^{-}, \mathcal{F}^{+}$, correspondingly. Consider the measurable space with measure $\left\{\Omega^{-} \times \Omega^{+}, \mathcal{F}^{-} \times \mathcal{F}^{+}, \mu\right\}$, which is a direct product of the measurable spaces with measures $\left\{\Omega^{-}, \mathcal{F}^{-}, P^{-}\right\}$and $\left\{\Omega^{+}, \mathcal{F}^{+}, P^{+}\right\}$, where $\mu=P^{-} \times P^{+}$. Introduce the denotations

$$
\begin{gathered}
\xi^{+}(\omega)= \begin{cases}\xi(\omega), & \omega \in\{\xi(\omega)>0\}, \\
0, & \omega \in\{\xi(\omega) \leq 0\},\end{cases} \\
\xi^{-}(\omega)= \begin{cases}-\xi(\omega), & \omega \in\{\xi(\omega) \leq 0\}, \\
0, & \omega \in\{\xi(\omega)>0\}\end{cases}
\end{gathered}
$$

Then, $\xi(\omega)=\xi^{+}(\omega)-\xi^{-}(\omega)$.

On the measurable space $\left\{\Omega^{-} \times \Omega^{+}, \mathcal{F}^{-} \times \mathcal{F}^{+}, P^{-} \times P^{+}\right\}$, we assume that the set of nonnegative measurable functions $\alpha\left(\omega_{1}, \omega_{2}\right)$, satisfying the conditions

$$
\begin{gathered}
\mu\left(\left\{\left(\omega_{1}, \omega_{2}\right) \in \Omega^{-} \times \Omega^{+}, \alpha\left(\omega_{1}, \omega_{2}\right)>0\right\}\right)=P\left(\Omega^{+}\right) P\left(\Omega^{-}\right), \\
\iint_{\Omega^{-}} \alpha\left(\omega_{1}, \omega_{2}\right) \frac{\xi^{-}\left(\omega_{1}\right) \xi^{+}\left(\omega_{2}\right)}{\xi^{-}\left(\omega_{1}\right)+\xi^{+}\left(\omega_{2}\right)} \mathrm{d} \mu\left(\omega_{1}, \omega_{2}\right)<\infty, \\
\iint_{\Omega^{-} \Omega^{+}} \alpha\left(\omega_{1}, \omega_{2}\right) \mathrm{d} \mu\left(\omega_{1}, \omega_{2}\right)=1,
\end{gathered}
$$


is a nonempty set. Such assumptions are true for the nonempty set of bounded random values $\alpha\left(\omega_{1}, \omega_{2}\right)$, for example, if the random value $\xi$ is an integrable one relative to the measure $P$.

Lemma 5. On the probability space $\{\Omega, \mathcal{F}, P\}$, let a random value $\xi$ satisfy the conditions (36) and let a measure $Q$ be equivalent to the measure $P$ and such that $E^{Q} \xi=0$. Then, for the measure $Q$ the following representation

$$
\begin{aligned}
Q(A)= & \int_{\Omega^{-} \Omega^{+}} \chi_{A}\left(\omega_{1}\right) \alpha\left(\omega_{1}, \omega_{2}\right) \frac{\xi^{+}\left(\omega_{2}\right)}{\xi^{-}\left(\omega_{1}\right)+\xi^{+}\left(\omega_{2}\right)} \mathrm{d} \mu\left(\omega_{1}, \omega_{2}\right) \\
& +\iint_{\Omega^{-} \Omega^{+}} \chi_{A}\left(\omega_{2}\right) \alpha\left(\omega_{1}, \omega_{2}\right) \frac{\xi^{-}\left(\omega_{1}\right)}{\xi^{-}\left(\omega_{1}\right)+\xi^{+}\left(\omega_{2}\right)} \mathrm{d} \mu\left(\omega_{1}, \omega_{2}\right), \quad A \in \mathcal{F},
\end{aligned}
$$

is valid for those random value $\alpha\left(\omega_{1}, \omega_{2}\right)$ that satisfy the conditions (39)-(41).

Every measure $Q$, given by the formula (42), with the random value $\alpha\left(\omega_{1}, \omega_{2}\right)$, satisfying the conditions (39)-(41) is equivalent to the measure $P$ and is such that $E^{Q} \xi=0$. For the measure $Q$, the canonical representation

$$
\begin{aligned}
Q(A)= & \iint_{\Omega^{-} \Omega^{+}} \chi_{A}\left(\omega_{1}\right) \alpha_{1}\left(\omega_{1}, \omega_{2}\right) \frac{\xi^{+}\left(\omega_{2}\right)}{\xi^{-}\left(\omega_{1}\right)+\xi^{+}\left(\omega_{2}\right)} \mathrm{d} \mu\left(\omega_{1}, \omega_{2}\right) \\
& +\iint_{\Omega^{-} \Omega^{+}} \chi_{A}\left(\omega_{2}\right) \alpha_{1}\left(\omega_{1}, \omega_{2}\right) \frac{\xi^{-}\left(\omega_{1}\right)}{\xi^{-}\left(\omega_{1}\right)+\xi^{+}\left(\omega_{2}\right)} \mathrm{d} \mu\left(\omega_{1}, \omega_{2}\right), \quad A \in \mathcal{F},
\end{aligned}
$$

is valid, where

$$
\begin{gathered}
\alpha_{1}\left(\omega_{1}, \omega_{2}\right)=\frac{\psi_{1}\left(\omega_{1}\right) \psi_{2}\left(\omega_{2}\right)\left[\xi^{-}\left(\omega_{1}\right)+\xi^{+}\left(\omega_{2}\right)\right]}{d},\left(\omega_{1}, \omega_{2}\right) \in \Omega^{-} \times \Omega^{+}, \\
\psi_{1}\left(\omega_{1}\right)=\int_{\Omega^{+}} \alpha\left(\omega_{1}, \omega_{2}\right) \frac{\xi^{+}\left(\omega_{2}\right)}{\xi^{-}\left(\omega_{1}\right)+\xi^{+}\left(\omega_{2}\right)} \mathrm{d} P\left(\omega_{2}\right), \omega_{1} \in \Omega^{-}, \\
\psi_{2}\left(\omega_{2}\right)=\int_{\Omega^{-}} \alpha\left(\omega_{1}, \omega_{2}\right) \frac{\xi^{-}\left(\omega_{1}\right)}{\xi^{-}\left(\omega_{1}\right)+\xi^{+}\left(\omega_{2}\right)} \mathrm{d} P\left(\omega_{1}\right), \omega_{2} \in \Omega^{+}, \\
d=\int_{\Omega^{-}} \xi^{-}\left(\omega_{1}\right) \psi_{1}\left(\omega_{1}\right) \mathrm{d} P\left(\omega_{1}\right)=\int_{\Omega^{+}} \xi^{+}\left(\omega_{2}\right) \psi_{2}\left(\omega_{2}\right) \mathrm{d} P\left(\omega_{2}\right) .
\end{gathered}
$$

Proof. From the Lemma 5 conditions,

$$
\begin{gathered}
Q(A)=\int_{A} \psi(\omega) \mathrm{d} P, P(\{\omega, \psi(\omega)>0\})=1, \\
\int_{\Omega} \psi(\omega) \xi(\omega) \mathrm{d} P(\omega)=0 .
\end{gathered}
$$

The condition (49) means

$$
\int_{\Omega^{+}} \psi_{2}\left(\omega_{2}\right) \xi^{+}\left(\omega_{2}\right) \mathrm{d} P\left(\omega_{2}\right)=\int_{\Omega^{-}} \psi_{1}\left(\omega_{1}\right) \xi^{-}\left(\omega_{1}\right) \mathrm{d} P\left(\omega_{1}\right)=d>0,
$$

where

$$
\psi_{1}(\omega)= \begin{cases}\psi(\omega), & \omega \in \Omega^{-} \\ 0, & \omega \in \Omega^{+}\end{cases}
$$




$$
\psi_{2}(\omega)= \begin{cases}\psi(\omega), & \omega \in \Omega^{+}, \\ 0, & \omega \in \Omega^{-}\end{cases}
$$

Let us put

$$
\alpha\left(\omega_{1}, \omega_{2}\right)=\frac{\psi_{1}\left(\omega_{1}\right) \psi_{2}\left(\omega_{2}\right)\left[\xi^{-}\left(\omega_{1}\right)+\xi^{+}\left(\omega_{2}\right)\right]}{d},\left(\omega_{1}, \omega_{2}\right) \in \Omega^{-} \times \Omega^{+} .
$$

Then, for such $\alpha\left(\omega_{1}, \omega_{2}\right)$ the equality (39) is true. Moreover,

$$
\begin{gathered}
\iint_{\Omega^{-} \Omega^{+}} \alpha\left(\omega_{1}, \omega_{2}\right) \frac{\xi^{-}\left(\omega_{1}\right) \xi^{+}\left(\omega_{2}\right)}{\xi^{-}\left(\omega_{1}\right)+\xi^{+}\left(\omega_{2}\right)} \mathrm{d} \mu\left(\omega_{1}, \omega_{2}\right)=d^{2}<\infty \\
\iint_{\Omega^{-} \Omega^{+}} \alpha\left(\omega_{1}, \omega_{2}\right) \mathrm{d} \mu\left(\omega_{1}, \omega_{2}\right)=\iint_{\Omega^{-}} \psi_{1}\left(\omega_{1}\right) \mathrm{d} P\left(\omega_{1}\right)+\int \psi_{\Omega^{+}} \psi_{2}\left(\omega_{2}\right) \mathrm{d} P\left(\omega_{2}\right)=1, \\
E^{Q} \xi=\iint_{\Omega^{-} \Omega^{+}} \alpha\left(\omega_{1}, \omega_{2}\right) \xi\left(\omega_{1}\right) \frac{\xi^{+}\left(\omega_{2}\right)}{\xi^{-}\left(\omega_{1}\right)+\xi^{+}\left(\omega_{2}\right)} \mathrm{d} \mu\left(\omega_{1}, \omega_{2}\right) \\
+\int_{\Omega^{-} \Omega^{+}} \alpha\left(\omega_{1}, \omega_{2}\right) \xi\left(\omega_{2}\right) \frac{\xi^{-}\left(\omega_{1}\right)}{\xi^{-}\left(\omega_{1}\right)+\xi^{+}\left(\omega_{2}\right)} \mathrm{d} \mu\left(\omega_{1}, \omega_{2}\right)=0, \\
\text { since } \xi\left(\omega_{1}\right)=-\xi^{-}\left(\omega_{1}\right), \omega_{1} \in \Omega^{-}, \xi\left(\omega_{2}\right)=\xi^{+}\left(\omega_{2}\right), \omega_{2} \in \Omega^{+}
\end{gathered}
$$

Let us prove the last statement of Lemma 5. Suppose that the representation (42) for the measure $Q$, satisfying the conditions (39)-(41), is valid. Taking into account the denotations (45)-(47), we obtain

$$
\begin{gathered}
Q(A)=\int_{\Omega^{-}} \chi_{A}\left(\omega_{1}\right) \psi_{1}\left(\omega_{1}\right) \mathrm{d} P\left(\omega_{1}\right)+\int_{\Omega^{+}} \chi_{A}\left(\omega_{2}\right) \psi_{2}\left(\omega_{2}\right) \mathrm{d} P\left(\omega_{2}\right), \\
0=E^{Q} \xi=\int_{\Omega^{-}} \xi\left(\omega_{1}\right) \psi_{1}\left(\omega_{1}\right) \mathrm{d} P\left(\omega_{1}\right)+\int_{\Omega^{+}} \xi\left(\omega_{2}\right) \psi_{2}\left(\omega_{2}\right) \mathrm{d} P\left(\omega_{2}\right) \\
=-\int_{\Omega^{-}} \xi^{-}\left(\omega_{1}\right) \psi_{1}\left(\omega_{1}\right) \mathrm{d} P\left(\omega_{1}\right)+\int_{\Omega^{+}} \xi^{+}\left(\omega_{2}\right) \psi_{2}\left(\omega_{2}\right) \mathrm{d} P\left(\omega_{2}\right) .
\end{gathered}
$$

If to introduce the denotation

$$
\psi(\omega)= \begin{cases}\psi_{1}(\omega), & \omega \in \Omega^{-}, \\ \psi_{2}(\omega), & \omega \in \Omega^{+},\end{cases}
$$

then we obtain the representation

$$
Q(A)=\int_{A} \psi(\omega) \mathrm{d} P(\omega)
$$

where $P\left(\psi_{1}(\omega)>0\right)=P\left(\Omega^{-}\right), P\left(\psi_{2}(\omega)>0\right)=P\left(\Omega^{+}\right)$.

The last formula proves the equivalence of the measures $Q$ and $P$. At last, to prove the canonical representation (43) it is sufficient to substitute the expression (44) for $\alpha_{1}\left(\omega_{1}, \omega_{2}\right)$ into the expression (43) for $Q(A)$. We obtain the expression (57) for $Q(A)$. Then, if to substitute the expressions (45), (46) for $\psi_{1}\left(\omega_{1}\right), \psi_{2}\left(\omega_{2}\right)$ into the expression (57) for $Q(A)$, we obtain that the canonical representation for $Q(A)$ is true. This proves Lemma 5 .

For further investigations, the next Theorem 4 is very important [5].

Theorem 4. The necessary and sufficient conditions of the local regularity of 
the nonnegative super-martingale $\left\{f_{m}, \mathcal{F}_{m}\right\}_{m=0}^{\infty}$ relative to a set of equivalent measures $M$ are the existence of $\mathcal{F}_{m}$-measurable random values $\xi_{m}^{0} \in A_{0}, m=\overline{1, \infty}$, such that

$$
\frac{f_{m}}{f_{m-1}} \leq \xi_{m}^{0}, E^{P}\left\{\xi_{m}^{0} \mid \mathcal{F}_{m-1}\right\}=1, P \in M, m=\overline{1, \infty}
$$

Proof. The necessity. Without loss of generality, we assume that $f_{m} \geq a$ for a certain real number $a>0$. Really, if it is not so, then we can come to the consideration of the super-martingale $\left\{f_{m}+a, \mathcal{F}_{m}\right\}_{m=0}^{\infty}$. Thus, let $\left\{f_{m}, \mathcal{F}_{m}\right\}_{m=0}^{\infty}$ be a nonnegative local regular super-martingale. Then, there exists a nonnegative adapted random process $\left\{g_{m}\right\}_{m=0}^{\infty}, g_{0}=0$, such that $\sup _{P \in M} E^{P} g_{m}<\infty$,

$$
f_{m-1}-E^{P}\left\{f_{m} \mid \mathcal{F}_{m-1}\right\}=E^{P}\left\{g_{m} \mid \mathcal{F}_{m-1}\right\}, P \in M, m=\overline{1, \infty} \text {. }
$$

Let us put $\xi_{m}^{0}=\frac{f_{m}+g_{m}}{f_{m-1}}, m=\overline{1, \infty}$. Then, $\xi_{m}^{0} \in A_{0}$ and from the equalities (62) we obtain $E^{P}\left\{\xi_{m}^{0} \mid \mathcal{F}_{m-1}\right\}=1, P \in M, m=\overline{1, \infty}$. It is evident that the inequalities (61) are valid.

The sufficiency. Suppose that the conditions of Theorem 4 are valid. Then, $f_{m} \leq f_{m-1}+f_{m-1}\left(\xi_{m}^{0}-1\right)$. Introduce the denotation $g_{m}=-f_{m}+f_{m-1} \xi_{m}^{0}$. Then, $g_{m} \geq 0, \sup _{P \in M} E^{P} g_{m} \leq \sup _{P \in M} E^{P} f_{m}+\sup _{P \in M} E^{P} f_{m-1}<\infty, m=\overline{1, \infty}$. The last equality and the inequalities give

$$
f_{m}=f_{0}+\sum_{i=1}^{m} f_{i-1}\left(\xi_{i}^{0}-1\right)-\sum_{i=1}^{m} g_{i}, \quad m=\overline{1, \infty} .
$$

Let us consider the random process $\left\{M_{m}, \mathcal{F}_{m}\right\}_{m=0}^{\infty}$, where $M_{m}=f_{0}+\sum_{i=1}^{m} f_{i-1}\left(\xi_{i}^{0}-1\right)$. Then, $E^{P}\left\{M_{m} \mid \mathcal{F}_{m-1}\right\}=M_{m-1}, P \in M, m=\overline{1, \infty}$. Theorem 4 is proved.

\section{Construction of the Regular Set of Measures}

In the next two Lemmas, we investigate the closure of a convex set of equivalent measures presented in Lemma 5 by the formula (42). First, we consider the countable case.

Suppose that $\Omega_{1}$ contains the countable set of elementary events and let $\mathcal{F}_{1}$ be a $\sigma$-algebra of all subsets of the set $\Omega_{1}$. Let $P_{1}$ be a measure on the $\sigma$-algebra $\mathcal{F}_{1}$. We assume that $P_{1}\left(\omega_{i}\right)=p_{i}>0, i=\overline{1, \infty}$. On the probability space $\left\{\Omega_{1}, \mathcal{F}_{1}, P_{1}\right\}$, let us consider a nonnegative random value $\xi_{1}$, satisfying the conditions

$$
\begin{gathered}
0<P_{1}\left(\left\{\omega \in \Omega_{1}, \eta_{1}(\omega)<0\right\}\right)<1,0<P_{1}\left(\left\{\omega \in \Omega_{1}, \eta_{1}(\omega)>0\right\}\right), \\
E^{P_{1}}\left|\eta_{1}(\omega)\right|<\infty,
\end{gathered}
$$

where we introduced the denotation $\eta_{1}(\omega)=\xi_{1}(\omega)-1$. On the measurable space $\left\{\Omega_{1}, \mathcal{F}_{1}\right\}$, let us consider the set of measures $M_{1}$, which are equivalent to the measure $P_{1}$ and are given by the formula 


$$
\begin{aligned}
& Q(A)=\sum_{\omega_{1} \in \Omega_{1}^{-}} \sum_{\omega_{2} \in \Omega_{1}^{+}} \chi_{A}\left(\omega_{1}\right) \alpha\left(\omega_{1}, \omega_{2}\right) \frac{\eta_{1}^{+}\left(\omega_{2}\right)}{\eta_{1}^{-}\left(\omega_{1}\right)+\eta_{1}^{+}\left(\omega_{2}\right)} P_{1}\left(\omega_{1}\right) P_{1}\left(\omega_{2}\right) \\
& +\sum_{\omega_{1} \in \Omega_{1}^{-}} \sum_{\omega_{2} \in \Omega_{1}^{+}} \chi_{A}\left(\omega_{2}\right) \alpha\left(\omega_{1}, \omega_{2}\right) \frac{\eta_{1}^{-}\left(\omega_{1}\right)}{\eta_{1}^{-}\left(\omega_{1}\right)+\eta_{1}^{+}\left(\omega_{2}\right)} P_{1}\left(\omega_{1}\right) P_{1}\left(\omega_{2}\right), A \in \mathcal{F}_{1},
\end{aligned}
$$

where $\eta_{1}(\omega)=\eta_{1}^{+}(\omega)-\eta_{1}^{-}(\omega), \quad \Omega_{1}^{+}=\left\{\omega, \eta_{1}(\omega)>0\right\}, \quad \Omega_{1}^{-}=\left\{\omega, \eta_{1}(\omega) \leq 0\right\}$. Introduce the denotations $\mathcal{F}_{1}^{+}=\Omega_{1}^{+} \cap \mathcal{F}_{1}, \mathcal{F}_{1}^{-}=\Omega_{1}^{-} \cap \mathcal{F}_{1}$. Let $P_{1}^{-}$be a contraction of the measure $P_{1}$ on the $\sigma$-algebra $\mathcal{F}_{1}^{-}$and let $P_{1}^{+}$be a contraction of the measure $P_{1}$ on the $\sigma$-algebra $\mathcal{F}_{1}^{+}$. On the probability space $\left\{\Omega_{1}^{-} \times \Omega_{1}^{+}, \mathcal{F}_{1}^{-} \times \mathcal{F}_{1}^{+}, P_{1}^{-} \times P_{1}^{+}\right\}$, the set of random value $\alpha\left(\omega_{1}, \omega_{2}\right)$ satisfies the conditions

$$
\begin{gathered}
P_{1}^{-} \times P_{1}^{+}\left(\left\{\left(\omega_{1}, \omega_{2}\right) \in \Omega_{1}^{-} \times \Omega_{1}^{+}, \alpha\left(\omega_{1}, \omega_{2}\right)>0\right\}\right)=P_{1}\left(\Omega_{1}^{+}\right) P_{1}\left(\Omega_{1}^{-}\right), \\
\sum_{\omega_{1} \in \Omega^{-}} \sum_{\omega_{2} \in \Omega^{+}} \alpha\left(\omega_{1}, \omega_{2}\right) \frac{\eta_{1}^{-}\left(\omega_{1}\right) \eta_{1}^{+}\left(\omega_{2}\right)}{\eta_{1}^{-}\left(\omega_{1}\right)+\eta_{1}^{+}\left(\omega_{2}\right)} P_{1}\left(\omega_{1}\right) P_{1}\left(\omega_{2}\right)<\infty, \\
\sum_{\omega_{1} \in \Omega_{1}^{-}} \sum_{\omega_{2} \in \Omega_{1}^{+}} \alpha\left(\omega_{1}, \omega_{2}\right) P_{1}\left(\omega_{1}\right) P_{1}\left(\omega_{2}\right)=1 .
\end{gathered}
$$

On the probability space $\left\{\Omega_{1}^{-} \times \Omega_{1}^{+}, \mathcal{F}_{1}^{-} \times \mathcal{F}_{1}^{+}, P_{1}^{-} \times P_{1}^{+}\right\}$, all the bounded strictly positive random values $\alpha\left(\omega_{1}, \omega_{2}\right)$ the above conditions satisfy. Introduce into the set of all measures on $\left\{\Omega_{1}, \mathcal{F}_{1}\right\}$ the metrics

$$
\rho\left(Q_{1}, Q_{2}\right)=\sum_{i=1}^{\infty}\left|Q_{1}\left(\omega_{i}\right)-Q_{2}\left(\omega_{i}\right)\right|
$$

Lemma 6. The closure of the set of measures $M_{1}$ in metrics (69) contains the set of measures

$$
v_{\omega_{1}, \omega_{2}}(A)=\chi_{A}\left(\omega_{1}\right) \frac{\eta_{1}^{+}\left(\omega_{2}\right)}{\eta_{1}^{-}\left(\omega_{1}\right)+\eta_{1}^{+}\left(\omega_{2}\right)}+\chi_{A}\left(\omega_{2}\right) \frac{\eta_{1}^{-}\left(\omega_{1}\right)}{\eta_{1}^{-}\left(\omega_{1}\right)+\eta_{1}^{+}\left(\omega_{2}\right)}
$$

for $\omega_{1} \in \Omega_{1}^{-}, \omega_{2} \in \Omega_{1}^{+}, A \in \mathcal{F}_{1}$. For every bounded random value $f(\omega)$, the closure of the set of points $E^{Q} f, Q \in M_{1}$, in metrics $\rho(x, y)=|x-y|, x, y \in R^{1}$, contains the points $E^{v_{\omega_{1}, \omega_{2}}} f,\left(\omega_{1}, \omega_{2}\right) \in \Omega_{1}^{-} \times \Omega_{1}^{+}$.

Proof. Let us choose the set of equivalent measures $Q^{\varepsilon}$ defined by $\alpha^{\varepsilon}\left(\omega_{1}, \omega_{2}\right), 0<\varepsilon<1$, and given by the law:

$$
\begin{gathered}
\alpha^{\varepsilon}\left(\omega_{1}^{0}, \omega_{2}^{0}\right)=\frac{1-\varepsilon}{P_{1}\left(\omega_{1}^{0}\right) P_{1}\left(\omega_{2}^{0}\right)}, \omega_{1}^{0} \in \Omega_{1}^{-}, \omega_{2}^{0} \in \Omega_{1}^{+}, \\
\alpha^{\varepsilon}\left(\omega_{1}, \omega_{2}\right)=\varepsilon \alpha_{0}^{\varepsilon}\left(\omega_{1}, \omega_{2}\right), \\
\alpha_{0}^{\varepsilon}\left(\omega_{1}, \omega_{2}\right)=\frac{1}{\sum_{\omega_{1} \neq \omega_{1}^{0} \omega \neq \omega_{2}^{0}} P\left(\omega_{1}\right) P\left(\omega_{2}\right)},\left(\omega_{1}, \omega_{2}\right) \neq\left(\omega_{1}^{0}, \omega_{2}^{0}\right), \\
\omega_{1} \in \Omega_{1}^{-}, \omega_{2} \in \Omega_{1}^{+} .
\end{gathered}
$$

It is evident that $\alpha^{\varepsilon}\left(\omega_{1}, \omega_{2}\right)>0,\left(\omega_{1}, \omega_{2}\right) \in \Omega_{1}^{-} \times \Omega_{1}^{+}$, for every $1>\varepsilon>0$, and satisfy the equality

$$
\sum_{\left(\omega_{1}, \omega_{2}\right) \in \Omega_{1}^{-} \times \Omega_{1}^{+}} \alpha^{\varepsilon}\left(\omega_{1}, \omega_{2}\right) P_{1}\left(\omega_{1}\right) P_{1}\left(\omega_{2}\right)=1
$$


Then,

$$
\begin{gathered}
Q^{\varepsilon}\left(\omega_{1}^{0}\right)=\sum_{\omega_{2} \in \Omega_{1}^{+}} \alpha^{\varepsilon}\left(\omega_{1}^{0}, \omega_{2}\right) \frac{\eta_{1}^{+}\left(\omega_{2}\right)}{\eta_{1}^{-}\left(\omega_{1}^{0}\right)+\eta_{1}^{+}\left(\omega_{2}\right)} P_{1}\left(\omega_{1}^{0}\right) P_{1}\left(\omega_{2}\right), \\
Q^{\varepsilon}\left(\omega_{2}^{0}\right)=\sum_{\omega_{1} \in \Omega_{1}^{-}} \alpha^{\varepsilon}\left(\omega_{1}, \omega_{2}^{0}\right) \frac{\eta_{1}^{-}\left(\omega_{1}\right)}{\eta_{1}^{-}\left(\omega_{1}\right)+\eta_{1}^{+}\left(\omega_{2}^{0}\right)} P_{1}\left(\omega_{1}\right) P_{1}\left(\omega_{2}^{0}\right) . \\
Q^{\varepsilon}\left(\omega_{1}^{0}\right)=(1-\varepsilon) \frac{\eta_{1}^{+}\left(\omega_{2}^{0}\right)}{\eta_{1}^{-}\left(\omega_{1}^{0}\right)+\eta_{1}^{+}\left(\omega_{2}^{0}\right)} \\
+\varepsilon \sum_{\omega_{2} \in \Omega_{1}^{+}, \omega_{2} \neq \omega_{2}^{0}} \alpha_{0}^{\varepsilon}\left(\omega_{1}^{0}, \omega_{2}\right) \frac{\eta_{1}^{+}\left(\omega_{2}\right)}{\eta_{1}^{-}\left(\omega_{1}^{0}\right)+\eta_{1}^{+}\left(\omega_{2}\right)} P_{1}\left(\omega_{1}^{0}\right) P_{1}\left(\omega_{2}\right), \\
Q^{\varepsilon}\left(\omega_{2}^{0}\right)= \\
\quad(1-\varepsilon) \frac{\eta_{1}^{-}\left(\omega_{1}^{0}\right)}{\eta_{1}^{-}\left(\omega_{1}^{0}\right)+\eta_{1}^{+}\left(\omega_{2}^{0}\right)} \\
+\varepsilon \sum_{\omega_{1} \in \Omega_{1}^{-}, \omega_{1} \neq \omega_{1}^{0}} \alpha_{0}^{\varepsilon}\left(\omega_{1}, \omega_{2}^{0}\right) \frac{\eta_{1}^{-}\left(\omega_{1}\right)}{\eta_{1}^{-}\left(\omega_{1}\right)+\eta_{1}^{+}\left(\omega_{2}^{0}\right)} P_{1}\left(\omega_{1}\right) P_{1}\left(\omega_{2}^{0}\right) .
\end{gathered}
$$

If $\omega_{1} \neq \omega_{1}^{0}, \omega_{2} \neq \omega_{2}^{0}$, then

$$
\begin{gathered}
Q^{\varepsilon}\left(\omega_{1}\right)=\varepsilon \sum_{\omega_{2} \in \Omega_{1}^{+}} \alpha_{0}^{\varepsilon}\left(\omega_{1}, \omega_{2}\right) \frac{\eta_{1}^{+}\left(\omega_{2}\right)}{\eta_{1}^{-}\left(\omega_{1}^{0}\right)+\eta_{1}^{+}\left(\omega_{2}\right)} P_{1}\left(\omega_{1}\right) P_{1}\left(\omega_{2}\right), \\
Q^{\varepsilon}\left(\omega_{2}\right)=\varepsilon \sum_{\omega_{1} \in \Omega_{1}^{-}} \alpha_{0}^{\varepsilon}\left(\omega_{1}, \omega_{2}\right) \frac{\eta_{1}^{-}\left(\omega_{1}\right)}{\eta_{1}^{-}\left(\omega_{1}\right)+\eta_{1}^{+}\left(\omega_{2}\right)} P_{1}\left(\omega_{1}\right) P_{1}\left(\omega_{2}\right) .
\end{gathered}
$$

The distance between the measures $Q^{\varepsilon}$ and $v_{\omega_{1}^{0}, \omega_{2}^{0}}$ is given by the formula

$$
\begin{aligned}
& \rho\left(Q^{\varepsilon}, v_{\omega_{1}^{0}, \omega_{2}^{0}}\right) \\
& =\varepsilon+\varepsilon \sum_{\omega_{2} \in \Omega_{1}^{+}, \omega_{2} \neq \omega_{2}^{0}} \alpha_{0}^{\varepsilon}\left(\omega_{1}^{0}, \omega_{2}\right) \frac{\eta_{1}^{+}\left(\omega_{2}\right)}{\eta_{1}^{-}\left(\omega_{1}^{0}\right)+\eta_{1}^{+}\left(\omega_{2}\right)} P_{1}\left(\omega_{1}^{0}\right) P_{1}\left(\omega_{2}\right) \\
& +\varepsilon \sum_{\omega_{1} \in \Omega_{1}^{-}, \omega_{1} \neq \omega_{1}^{0}} \alpha_{0}^{\varepsilon}\left(\omega_{1}, \omega_{2}^{0}\right) \frac{\eta_{1}^{-}\left(\omega_{1}\right)}{\eta_{1}^{-}\left(\omega_{1}\right)+\eta_{1}^{+}\left(\omega_{2}^{0}\right)} P_{1}\left(\omega_{1}\right) P_{1}\left(\omega_{2}^{0}\right) \\
& +\varepsilon \sum_{\omega_{1} \in \Omega_{1}^{-}, \omega_{1} \neq \omega_{1}^{0} \omega_{2} \in \Omega_{1}^{+}} \alpha_{0}^{\varepsilon}\left(\omega_{1}, \omega_{2}\right) \frac{\eta_{1}^{+}\left(\omega_{2}\right)}{\eta_{1}^{-}\left(\omega_{1}^{0}\right)+\eta_{1}^{+}\left(\omega_{2}\right)} P_{1}\left(\omega_{1}\right) P_{1}\left(\omega_{2}\right) \\
& +\varepsilon \sum_{\omega_{2} \in \Omega_{1}^{+}, \omega_{2} \neq \omega_{2}^{0} \omega_{1} \in \Omega_{1}^{-}} \alpha_{0}^{\varepsilon}\left(\omega_{1}, \omega_{2}\right) \frac{\eta_{1}^{-}\left(\omega_{1}\right)}{\eta_{1}^{-}\left(\omega_{1}\right)+\eta_{1}^{+}\left(\omega_{2}\right)} P_{1}\left(\omega_{1}\right) P_{1}\left(\omega_{2}\right) .
\end{aligned}
$$

Since

$$
\begin{gathered}
\sum_{\omega_{2} \in \Omega_{1}^{+}, \omega_{2} \neq \omega_{2}^{0}} \alpha_{0}^{\varepsilon}\left(\omega_{1}^{0}, \omega_{2}\right) \frac{\eta_{1}^{+}\left(\omega_{2}\right)}{\eta_{1}^{-}\left(\omega_{1}^{0}\right)+\eta_{1}^{+}\left(\omega_{2}\right)} P_{1}\left(\omega_{1}^{0}\right) P_{1}\left(\omega_{2}\right) \\
+\sum_{\omega_{1} \in \Omega_{1}^{-}, \omega_{1} \neq \omega_{1}^{0}} \alpha_{0}^{\varepsilon}\left(\omega_{1}, \omega_{2}^{0}\right) \frac{\eta_{1}^{-}\left(\omega_{1}\right)}{\eta_{1}^{-}\left(\omega_{1}\right)+\eta_{1}^{+}\left(\omega_{2}^{0}\right)} P_{1}\left(\omega_{1}\right) P_{1}\left(\omega_{2}^{0}\right) \leq 1, \\
\sum_{\omega_{1} \in \Omega_{1}^{-}, \omega_{1} \neq \omega_{1}^{0} \omega_{2} \in \Omega_{1}^{+}} \alpha_{0}^{\varepsilon}\left(\omega_{1}, \omega_{2}\right) \frac{\eta_{1}^{+}\left(\omega_{2}\right)}{\eta_{1}^{-}\left(\omega_{1}^{0}\right)+\eta_{1}^{+}\left(\omega_{2}\right)} P_{1}\left(\omega_{1}\right) P_{1}\left(\omega_{2}\right) \leq 1,
\end{gathered}
$$




$$
\sum_{\omega_{2} \in \Omega_{1}^{+}, \omega_{2} \neq \omega_{2}^{0} \omega_{1} \in \Omega_{1}^{-}} \alpha_{0}^{\varepsilon}\left(\omega_{1}, \omega_{2}\right) \frac{\eta_{1}^{-}\left(\omega_{1}\right)}{\eta_{1}^{-}\left(\omega_{1}\right)+\eta_{1}^{+}\left(\omega_{2}\right)} P_{1}\left(\omega_{1}\right) P_{1}\left(\omega_{2}\right) \leq 1,
$$

we obtain

$$
\rho\left(Q^{\varepsilon}, v_{\omega_{1}^{0}, \omega_{2}^{0}}\right) \leq 4 \varepsilon .
$$

Let us prove the second part of Lemma 6. It is evident that the inequality

$$
\left|E^{Q^{\varepsilon}} f-E^{v \omega_{1}^{0}, \omega_{2}^{0}} f\right| \leq 4 \varepsilon \sup _{\omega \in \Omega_{1}}|f(\omega)|
$$

is true. Due to arbitrariness of the small $\varepsilon$, Lemma 6 is proved.

Definition 4. Let $\left\{\Omega_{1}, \mathcal{F}_{1}\right\}$ be a measurable space. The decomposition $A_{n, k}, n, k=\overline{1, \infty}$, of the space $\Omega_{1}$ we call exhaustive one if the following conditions are valid:

1) $A_{n, k} \in \mathcal{F}_{1}, A_{n, k} \cap A_{n, s}=\varnothing, k \neq s, \bigcup_{k=1}^{\infty} A_{n, k}=\Omega_{1}, n=\overline{1, \infty}$;

2) the $(n+1)$-th decomposition is a sub-decomposition of the $n$-th one, that is, for every $j, A_{n+1, j} \subseteq A_{n, k}$ for a certain $k=k(j)$;

3) the minimal $\sigma$-algebra containing all $A_{n, k}, n, k=\overline{1, \infty}$, coincides with $\mathcal{F}_{1}$.

The next Remark 1 is important for the construction of the filtration having the exhaustive decomposition.

Remark 1. Suppose that the measurable spaces $\left\{\Omega_{1}, \mathcal{F}_{1}\right\}$ and $\left\{\Omega_{2}, \mathcal{F}_{2}\right\}$ have the exhaustive decompositions $A_{n, k}^{1}, n, k=\overline{1, \infty}$ and $A_{m, s}^{2}, m, s=\overline{1, \infty}$, respectively, then the measurable space $\left\{\Omega_{1} \times \Omega_{2}, \mathcal{F}_{1} \times \mathcal{F}_{2}\right\}$ also have the exhaustive decomposition $\quad B_{n, k s}, n=\overline{1, \infty}, k, s=\overline{1, \infty}$, $B_{n, k s}=A_{n, k}^{1} \times A_{n, s}^{2}, k, s=\overline{1, \infty}, n=\overline{1, \infty}$. Really,

1) $A_{n, k}^{1} \times A_{n, s}^{2} \in \mathcal{F}_{1} \times \mathcal{F}_{2}, A_{n, k}^{1} \times A_{n, s}^{2} \cap A_{n, t}^{1} \times A_{n, r}^{2}=\varnothing,(k, s) \neq(t, r)$,

$$
\bigcup_{k, s=1}^{\infty} B_{n, k s}=\Omega_{1} \times \Omega_{2}, n=\overline{1, \infty} \text {; }
$$

2) the $(n+1)$-th decomposition is a sub-decomposition of the $n$-th one, that is, for every $k, s \quad B_{n+1, k s} \subseteq B_{n, i j}$ for a certain $i=i(k), j=j(s)$;

3) the minimal $\sigma$-algebra containing all $B_{n, k s}, n, k, s=\overline{1, \infty}$, coincides with $F_{1} \times F_{2}$.

In the next Lemma we give the sufficient condition of the existence of exhaustive decomposition.

Lemma 7. Let $\left\{\Omega_{1}, \mathcal{F}_{1}\right\}$ be a measurable space with a complete separable metric space $\Omega_{1}$ and Borel $\sigma$-algebra $\mathcal{F}_{1}$ on it. Then $\left\{\Omega_{1}, \mathcal{F}_{1}\right\}$ has an exhaustive decomposition.

Proof. If $\left\{\omega_{1}, \cdots, \omega_{n}, \cdots\right\}$ is a countable dense set in $\Omega_{1}$, then we denote

$$
B\left(\omega_{n}, \varepsilon_{m}\right)=\left\{\omega \in \Omega_{1}, \rho\left(\omega, \omega_{n}\right)<\varepsilon_{m}\right\}, n, m=\overline{1, \infty},
$$

the countable set of open balls as $\varepsilon_{m}$ runs all positive rational numbers, where $\rho\left(\omega_{1}, \omega_{2}\right), \omega_{1}, \omega_{2} \in \Omega_{1}$ is a metric in $\Omega_{1}$. Prove that $\mathcal{F}_{1}=\sigma\left(B\left(\omega_{n}, \varepsilon_{m}\right), n, m=\overline{1, \infty}\right)$, where $\sigma\left(B\left(\omega_{n}, \varepsilon_{m}\right), n, m=\overline{1, \infty}\right)$ is a minimal $\sigma$-algebra generated by the sets (80). For this purpose let us prove that for every open set $A \in \Omega_{1}$ the representation 


$$
A=\bigcup_{n_{k} \in N_{1}, m_{s} \in Q_{+}^{1}} B\left(\omega_{n_{k}}, \varepsilon_{m_{s}}\right)
$$

is true, where $N_{1}$ is a subset of positive integers, and $Q_{+}^{1}$ is a subset of positive rational numbers. Let us denote $\left\{\omega_{1}^{A}, \cdots, \omega_{n}^{A}, \cdots\right\}=A \cap\left\{\omega_{1}, \cdots, \omega_{n}, \cdots\right\}$. Suppose that $\omega_{0} \in A$, then $d=\inf _{\omega \in \bar{A} \backslash A} \rho\left(\omega_{0}, \omega\right)>0$, where $\bar{A}$ is a closure of the set $A$. Let the point $\omega_{k_{0}}^{A}$ belong to the ball $C\left(\omega_{0}, \frac{d}{8}\right)=\left\{\omega \in \Omega_{1}, \rho\left(\omega_{0}, \omega\right)<\frac{d}{8}\right\}$ and let us consider the ball

$$
C\left(\omega_{k_{0}}, \frac{d}{8}+\rho\left(\omega_{0}, \omega_{k_{0}}^{A}\right)\right)=\left\{\omega \in \Omega_{1}, \rho\left(\omega_{k_{0}}, \omega\right)<\frac{d}{8}+\rho\left(\omega_{0}, \omega_{k_{0}}^{A}\right)\right\} \text {. The point } \omega_{0}
$$

belongs to this ball and for every $\omega \in C\left(\omega_{k_{0}}, \frac{d}{8}+\rho\left(\omega_{0}, \omega_{k_{0}}^{A}\right)\right)$ the inequality

$$
\rho\left(\omega_{0}, \omega\right) \leq \rho\left(\omega_{0}, \omega_{k_{0}}^{A}\right)+\rho\left(\omega_{k_{0}}^{A}, \omega\right)<\frac{d}{8}+2 \rho\left(\omega_{0}, \omega_{k_{0}}^{A}\right)<\frac{3 d}{8}
$$

is true. Therefore $C\left(\omega_{k_{0}}, \frac{d}{8}+\rho\left(\omega_{0}, \omega_{k_{0}}^{A}\right)\right) \subset C\left(\omega_{0}, \frac{3 d}{8}\right)$. Let the rational number $\varepsilon_{k_{0}}$ satisfies the inequalities

$$
\frac{d}{8}+2 \rho\left(\omega_{0}, \omega_{k_{0}}^{A}\right)<\varepsilon_{k_{0}}<\frac{3 d}{8},
$$

then $C\left(\omega_{k_{0}}^{A}, \varepsilon_{k_{0}}\right) \subseteq C\left(\omega_{0}, \frac{d}{2}\right)$, since for every $\omega \in C\left(\omega_{k_{0}}^{A}, \varepsilon_{k_{0}}\right)$, $\rho\left(\omega_{0}, \omega\right) \leq \rho\left(\omega_{0}, \omega_{k_{0}}\right)+\rho\left(\omega_{k_{0}}, \omega\right)<\frac{d}{8}+\varepsilon_{k_{0}}<\frac{d}{2}$. So, for $\omega_{0} \in A$ we found $\omega_{k_{0}} \in\left\{\omega_{1}, \cdots, \omega_{n}, \cdots\right\}$ and the rational number $\varepsilon_{k_{0}}$ such that $\omega_{0} \in C\left(\omega_{k_{0}}^{A}, \varepsilon_{k_{0}}\right) \subset C\left(\omega_{0}, \frac{d}{2}\right) \subset A$. The last prove the needed statement. To complete the proof of Lemma 7 let us construct the exhaustive decomposition. Let us renumber the sets $B\left(\omega_{n}, \varepsilon_{m}\right)$ putting by $D_{1}=B\left(\omega_{1}, \varepsilon_{1}\right)$, $D_{2}=B\left(\omega_{1}, \varepsilon_{2}\right), D_{3}=B\left(\omega_{2}, \varepsilon_{1}\right)$, and so on. We put that $\left\{A_{1 k}\right\}_{k=1}^{\infty}$ consists of two sets $D_{1}$ and $\bar{D}_{1}=\Omega_{1} \backslash D_{1}$. If the set $\left\{A_{n k}\right\}_{k=1}^{\infty}$ is constructed, then the set $\left\{A_{n+1 k}\right\}_{k=1}^{\infty}$ we construct from the various set of the kind $A_{n k} \cap D_{n+1}, A_{n k} \cap \bar{D}_{n+1}$. By construction the minimal $\sigma$-algebra

$\sigma\left\{A_{n k}, n, k=\overline{1, \infty}\right\}=\sigma\left\{B\left(\omega_{n}, \varepsilon_{m}\right), n, m=\overline{1, \infty}\right\}$. Taking into account the previous part of the proof we have $\sigma\left\{A_{n k}, n, k=\overline{1, \infty}\right\}=\mathcal{F}_{1}$. Lemma 7 is proved.

Lemma 8. Let a measurable space $\{\Omega, \mathcal{F}\}$ have an exhaustive decomposition and let $\xi$ be an integrable random value relative to the measure $P$, satisfying the conditions (36). Then, the closure of the set of measure $Q$, given by the formula (42), relative to the pointwise convergence of measures contains the set of measures

$$
\begin{aligned}
v_{\omega_{1}, \omega_{2}}(A)= & \chi_{A}\left(\omega_{1}\right) \frac{\xi^{+}\left(\omega_{2}\right)}{\xi^{-}\left(\omega_{1}\right)+\xi^{+}\left(\omega_{2}\right)} \\
& +\chi_{A}\left(\omega_{2}\right) \frac{\xi^{-}\left(\omega_{1}\right)}{\xi^{-}\left(\omega_{1}\right)+\xi^{+}\left(\omega_{2}\right)}, A \in \mathcal{F},\left(\omega_{1}, \omega_{2}\right) \in \Omega^{-} \times \Omega^{+},
\end{aligned}
$$


for those $\left(\omega_{1}, \omega_{2}\right) \in \Omega^{-} \times \Omega^{+}$which have the full measure $\mu=P^{-} \times P^{+}$For every integrable finite valued random value $f(\omega)$ relative to all measures $Q$, the closure in metrics $\rho\left(x_{1}, x_{2}\right)=\left|x_{1}-x_{2}\right|, x_{1}, x_{2} \in R^{1}$, of the set of real numbers

$$
\begin{aligned}
E^{Q} f= & \int_{\Omega^{-} \Omega^{+}} f\left(\omega_{1}\right) \alpha\left(\omega_{1}, \omega_{2}\right) \frac{\xi^{+}\left(\omega_{2}\right)}{\xi^{-}\left(\omega_{1}\right)+\xi^{+}\left(\omega_{2}\right)} \mathrm{d} \mu\left(\omega_{1}, \omega_{2}\right) \\
& +\int_{\Omega^{-} \Omega^{+}} f\left(\omega_{2}\right) \alpha\left(\omega_{1}, \omega_{2}\right) \frac{\xi^{-}\left(\omega_{1}\right)}{\xi^{-}\left(\omega_{1}\right)+\xi^{+}\left(\omega_{2}\right)} \mathrm{d} \mu\left(\omega_{1}, \omega_{2}\right),
\end{aligned}
$$

when $\alpha\left(\omega_{1}, \omega_{2}\right)$ runs over all random values satisfying the conditions (39), (41), contains the set of numbers

$$
f\left(\omega_{1}\right) \frac{\xi^{+}\left(\omega_{2}\right)}{\xi^{-}\left(\omega_{1}\right)+\xi^{+}\left(\omega_{2}\right)}+f\left(\omega_{2}\right) \frac{\xi^{-}\left(\omega_{1}\right)}{\xi^{-}\left(\omega_{1}\right)+\xi^{+}\left(\omega_{2}\right)},\left(\omega_{1}, \omega_{2}\right) \in \Omega^{-} \times \Omega^{+} .
$$

Proof. On a probability space $\{\Omega, \mathcal{F}, P\}$, let $\xi$ be an integrable random value, satisfying the conditions (36). As before, let $\Omega^{+}=\{\omega, \xi(\omega)>0\}$, $\Omega^{-}=\{\omega, \xi(\omega) \leq 0\}$ and let $\mathcal{F}^{-}, \mathcal{F}^{+}$be the restrictions of the $\sigma$-algebra $\mathcal{F}$ on the sets $\Omega^{-}$and $\Omega^{+}$, correspondingly. Suppose that $P^{-}$and $P^{+}$are the contractions of the measure $P$ on the $\sigma$-algebras $\mathcal{F}^{-}, \mathcal{F}^{+}$, correspondingly. Consider the probability space $\left\{\Omega^{-} \times \Omega^{+}, \mathcal{F}^{-} \times \mathcal{F}^{+}, P^{-} \times P^{+}\right\}$which is a direct product of the probability spaces $\left\{\Omega^{-}, \mathcal{F}^{-}, P^{-}\right\}$and $\left\{\Omega^{+}, \mathcal{F}^{+}, P^{+}\right\}$. Due to Lemma 8 conditions and Remark 1, the measurable space $\left\{\Omega^{-} \times \Omega^{+}, \mathcal{F}^{-} \times \mathcal{F}^{+}\right\}$ has the exhaustive decomposition $B_{n, k s}, k, s=\overline{1, \infty}, n=\overline{1, \infty}$. Denote $\mathcal{F}_{n}$ the minimal $\sigma$-algebra generated by decomposition $B_{n, k s}, k, s=\overline{1, \infty}$. It is evident that $\mathcal{F}_{n} \subset \mathcal{F}_{n+1}$. Moreover, $\sigma\left(\bigvee_{n=1}^{\infty} \mathcal{F}_{n}\right)=\mathcal{F}^{-} \times \mathcal{F}^{+}$. On the probability space $\left\{\Omega^{-} \times \Omega^{+}, \mathcal{F}^{-} \times \mathcal{F}^{+}, P^{-} \times P^{+}\right\}$, for every integrable finite valued random value $f\left(\omega_{1}, \omega_{2}\right)$ the sequence $E^{\mu}\left\{f\left(\omega_{1}, \omega_{2}\right) \mid \mathcal{F}_{n}\right\}$ converges to $f\left(\omega_{1}, \omega_{2}\right)$ with probability one, as $n \rightarrow \infty$, since it is a regular martingale. It is evident that for those $B_{n, k s}$ for which $\mu\left(B_{n, k s}\right) \neq 0$

$$
E^{\mu}\left\{f\left(\omega_{1}, \omega_{2}\right) \mid \mathcal{F}_{n}\right\}=\frac{\int_{B_{n, k s}} f\left(\omega_{1}, \omega_{2}\right) \mathrm{d} \mu}{\mu\left(B_{n, k s}\right)},\left(\omega_{1}, \omega_{2}\right) \in B_{n, k s} .
$$

Denote $D_{0}=\bigcup_{n, k, s, \mu\left(B_{n, k s}\right)=0} B_{n, k s}$. It is evident that $\mu\left(D_{0}\right)=0$. For every $\left(\omega_{1}, \omega_{2}\right) \in \Omega^{-} \times \Omega^{+} \backslash D_{0}$, the formula (87) is well defined and is finite. Let $D_{1}$ be the subset of the set $\Omega^{-} \times \Omega^{+} \backslash D_{0}$, where the limit of the left hand side of the formula (87) does not exist. Then, $\mu\left(D_{1}\right)=0$. For every $\left(\omega_{1}, \omega_{2}\right) \in \Omega^{-} \times \Omega^{+} \backslash\left(D_{0} \cup D_{1}\right)$, the right hand side of the formula (87) converges to $f\left(\omega_{1}, \omega_{2}\right)$. For $\left(\omega_{1}, \omega_{2}\right) \in \Omega^{-} \times \Omega^{+} \backslash\left(D_{0} \cup D_{1}\right)$, denote $A_{n}=A_{n}\left(\omega_{1}, \omega_{2}\right)$ those set $B_{n, k s}$ for which $\left(\omega_{1}, \omega_{2}\right) \in B_{n, k s}$ for a certain $k, s$. Then, for every integrable finite valued $f\left(\omega_{1}, \omega_{2}\right)$ 


$$
\lim _{n \rightarrow \infty} \frac{\int_{A_{n}} f\left(\omega_{1}, \omega_{2}\right) \mathrm{d} \mu}{\mu\left(A_{n}\right)}=f\left(\omega_{1}, \omega_{2}\right) .
$$

Let us consider the sequence

$$
\alpha_{n}^{\varepsilon_{n}}\left(\omega_{1}, \omega_{2}\right)=\left(1-\varepsilon_{n}\right) \frac{\chi_{A_{n}}\left(\omega_{1}, \omega_{2}\right)}{\mu\left(A_{n}\right)}+\varepsilon_{n} \frac{\chi_{\Omega^{-} \times \Omega^{+} \backslash A_{n}}\left(\omega_{1}, \omega_{2}\right)}{\mu\left(\Omega^{-} \times \Omega^{+} \backslash A_{n}\right)},
$$

where $0<\varepsilon_{n}<1, \lim \varepsilon_{n}=0$. Such a sequence $\alpha_{n}^{\varepsilon_{n}}\left(\omega_{1}, \omega_{2}\right)$ satisfies the conditions (39)-(41) and

$$
\begin{aligned}
& Q_{n}^{\varepsilon_{n}}(A)=\iint_{\Omega^{-} \Omega^{+}} \chi_{A}\left(\omega_{1}\right) \alpha_{n}^{\varepsilon_{n}}\left(\omega_{1}, \omega_{2}\right) \frac{\xi^{+}\left(\omega_{2}\right)}{\xi^{-}\left(\omega_{1}\right)+\xi^{+}\left(\omega_{2}\right)} \mathrm{d} \mu\left(\omega_{1}, \omega_{2}\right) \\
& +\iint_{\Omega^{-} \Omega^{+}} \chi_{A}\left(\omega_{2}\right) \alpha_{n}^{\varepsilon_{n}}\left(\omega_{1}, \omega_{2}\right) \frac{\xi^{-}\left(\omega_{1}\right)}{\xi^{-}\left(\omega_{1}\right)+\xi^{+}\left(\omega_{2}\right)} \mathrm{d} \mu\left(\omega_{1}, \omega_{2}\right) \\
& =\left(1-\varepsilon_{n}\right) \frac{\int A_{n} \chi_{A}\left(\omega_{1}\right) \frac{\xi^{+}\left(\omega_{2}\right)}{\xi^{-}\left(\omega_{1}\right)+\xi^{+}\left(\omega_{2}\right)} \mathrm{d} \mu\left(\omega_{1}, \omega_{2}\right)}{\mu\left(A_{n}\right)} \\
& +\left(1-\varepsilon_{n}\right) \frac{\int_{A_{n}} \chi_{A}\left(\omega_{2}\right) \frac{\xi^{-}\left(\omega_{1}\right)}{\xi^{-}\left(\omega_{1}\right)+\xi^{+}\left(\omega_{2}\right)} \mathrm{d} \mu\left(\omega_{1}, \omega_{2}\right)}{\mu\left(A_{n}\right)} \\
& +\varepsilon_{n} \frac{\int_{\Omega^{-} \times \Omega^{+} \backslash A_{n}} \chi_{A}\left(\omega_{1}\right) \frac{\xi^{+}\left(\omega_{2}\right)}{\xi^{-}\left(\omega_{1}\right)+\xi^{+}\left(\omega_{2}\right)} \mathrm{d} \mu\left(\omega_{1}, \omega_{2}\right)}{\mu\left(\Omega^{-} \times \Omega^{+} \backslash A_{n}\right)} \\
& +\varepsilon_{n} \frac{\int_{\Omega^{-} \times \Omega^{+} \backslash A_{n}} \chi_{A}\left(\omega_{2}\right) \frac{\xi^{-}\left(\omega_{1}\right)}{\xi^{-}\left(\omega_{1}\right)+\xi^{+}\left(\omega_{2}\right)} \mathrm{d} \mu\left(\omega_{1}, \omega_{2}\right)}{\mu\left(\Omega^{-} \times \Omega^{+} \backslash A_{n}\right)} .
\end{aligned}
$$

From the formula (90), we obtain

$$
\begin{aligned}
& \lim _{n \rightarrow \infty} Q_{n}^{\varepsilon_{n}}(A)=\chi_{A}\left(\omega_{1}\right) \frac{\xi^{+}\left(\omega_{2}\right)}{\xi^{-}\left(\omega_{1}\right)+\xi^{+}\left(\omega_{2}\right)}+\chi_{A}\left(\omega_{2}\right) \frac{\xi^{-}\left(\omega_{1}\right)}{\xi^{-}\left(\omega_{1}\right)+\xi^{+}\left(\omega_{2}\right)}, \\
& A \in \mathcal{F},\left(\omega_{1}, \omega_{2}\right) \in \Omega^{-} \times \Omega^{+} \backslash\left(D_{0} \cup D_{1}\right) .
\end{aligned}
$$

Further,

$$
\begin{aligned}
E^{Q_{n}^{\varepsilon_{n}}} f(\omega)= & \iint_{\Omega^{-} \Omega^{+}} f\left(\omega_{1}\right) \alpha_{n}^{\varepsilon_{n}}\left(\omega_{1}, \omega_{2}\right) \frac{\xi^{+}\left(\omega_{2}\right)}{\xi^{-}\left(\omega_{1}\right)+\xi^{+}\left(\omega_{2}\right)} \mathrm{d} \mu\left(\omega_{1}, \omega_{2}\right) \\
& +\iint_{\Omega^{-} \Omega^{+}} f\left(\omega_{2}\right) \alpha_{n}^{\varepsilon_{n}}\left(\omega_{1}, \omega_{2}\right) \frac{\xi^{-}\left(\omega_{1}\right)}{\xi^{-}\left(\omega_{1}\right)+\xi^{+}\left(\omega_{2}\right)} \mathrm{d} \mu\left(\omega_{1}, \omega_{2}\right) \\
= & \left(1-\varepsilon_{n}\right) \frac{\int_{A_{n}} f\left(\omega_{1}\right) \frac{\xi^{+}\left(\omega_{2}\right)}{\xi^{-}\left(\omega_{1}\right)+\xi^{+}\left(\omega_{2}\right)} \mathrm{d} \mu\left(\omega_{1}, \omega_{2}\right)}{\mu\left(A_{n}\right)} \\
& +\left(1-\varepsilon_{n}\right) \frac{\int_{\frac{A_{n}}{A_{n}} f\left(\omega_{2}\right) \frac{\xi^{-}\left(\omega_{1}\right)}{\xi^{-}\left(\omega_{1}\right)+\xi^{+}\left(\omega_{2}\right)} \mathrm{d} \mu\left(\omega_{1}, \omega_{2}\right)}^{\mu\left(A_{n}\right)}}{}
\end{aligned}
$$




$$
\begin{gathered}
+\varepsilon_{n} \frac{\int_{\Omega^{-} \times \Omega^{+} \backslash A_{n}} f\left(\omega_{1}\right) \frac{\xi^{+}\left(\omega_{2}\right)}{\xi^{-}\left(\omega_{1}\right)+\xi^{+}\left(\omega_{2}\right)} \mathrm{d} \mu\left(\omega_{1}, \omega_{2}\right)}{\mu\left(\Omega^{-} \times \Omega^{+} \backslash A_{n}\right)} \\
+\varepsilon_{n} \frac{\int_{\Omega^{-} \times \Omega^{+} \backslash A_{n}} f\left(\omega_{2}\right) \frac{\xi^{-}\left(\omega_{1}\right)}{\xi^{-}\left(\omega_{1}\right)+\xi^{+}\left(\omega_{2}\right)} \mathrm{d} \mu\left(\omega_{1}, \omega_{2}\right)}{\mu\left(\Omega^{-} \times \Omega^{+} \backslash A_{n}\right)} .
\end{gathered}
$$

From the formula (92), we obtain

$$
\begin{aligned}
& \lim _{n \rightarrow \infty} E^{Q_{n}^{\varepsilon_{n}}} f(\omega)=f\left(\omega_{1}\right) \frac{\xi^{+}\left(\omega_{2}\right)}{\xi^{-}\left(\omega_{1}\right)+\xi^{+}\left(\omega_{2}\right)}+f\left(\omega_{2}\right) \frac{\xi^{-}\left(\omega_{1}\right)}{\xi^{-}\left(\omega_{1}\right)+\xi^{+}\left(\omega_{2}\right)}, \\
& \left(\omega_{1}, \omega_{2}\right) \in \Omega^{-} \times \Omega^{+} \backslash\left(D_{0} \cup D_{1}\right) .
\end{aligned}
$$

Lemma 8 is proved.

The next Theorem 5 is a consequence of Lemma 5 .

Theorem 5. On the probability space $\{\Omega, \mathcal{F}, P\}$, for the nonnegative random value $\xi \neq 1$ the set of measures $M_{0}$ on the measurable space $\{\Omega, \mathcal{F}\}$, being equivalent to the measure $P$, satisfies the condition

$$
E^{Q} \xi=1, Q \in M_{0},
$$

if and only if as for $Q \in M_{0}$ the representation

$$
\begin{aligned}
& Q(A)=\iint_{\Omega^{-} \Omega^{+}} \chi_{A}\left(\omega_{1}\right) \alpha\left(\omega_{1}, \omega_{2}\right) \frac{(\xi-1)^{+}\left(\omega_{2}\right)}{(\xi-1)^{-}\left(\omega_{1}\right)+(\xi-1)^{+}\left(\omega_{2}\right)} \mathrm{d} \mu\left(\omega_{1}, \omega_{2}\right) \\
& +\int_{\Omega^{-} \Omega^{+}} \chi_{A}\left(\omega_{2}\right) \alpha\left(\omega_{1}, \omega_{2}\right) \frac{(\xi-1)^{-}\left(\omega_{1}\right)}{(\xi-1)^{-}\left(\omega_{1}\right)+(\xi-1)^{+}\left(\omega_{2}\right)} \mathrm{d} \mu\left(\omega_{1}, \omega_{2}\right), \quad A \in \mathcal{F},
\end{aligned}
$$

is true, where on the measurable space $\left\{\Omega^{-} \times \Omega^{+}, \mathcal{F}^{-} \times \mathcal{F}^{+}, P^{-} \times P^{+}\right\}$, the random value $\alpha\left(\omega_{1}, \omega_{2}\right)$ satisfies the conditions

$$
\begin{gathered}
\mu\left(\left\{\left(\omega_{1}, \omega_{2}\right) \in \Omega^{-} \times \Omega^{+}, \alpha\left(\omega_{1}, \omega_{2}\right)>0\right\}\right)=P\left(\Omega^{+}\right) P\left(\Omega^{-}\right), \\
\iint_{\Omega^{-} \Omega^{+}} \alpha\left(\omega_{1}, \omega_{2}\right) \frac{(\xi-1)^{-}\left(\omega_{1}\right)(\xi-1)^{+}\left(\omega_{2}\right)}{(\xi-1)^{-}\left(\omega_{1}\right)+(\xi-1)^{+}\left(\omega_{2}\right)} \mathrm{d} \mu\left(\omega_{1}, \omega_{2}\right)<\infty, \\
\iint_{\Omega^{-} \Omega^{+}} \alpha\left(\omega_{1}, \omega_{2}\right) \mathrm{d} \mu\left(\omega_{1}, \omega_{2}\right)=1 .
\end{gathered}
$$

We introduced above the following denotations: $\mu=P^{-} \times P^{+}, P^{-}$is a contraction of the measure $P$ on the set $\Omega^{-}=\{\omega \in \Omega, \xi-1 \leq 0\}, P^{+}$is a contraction of the measure $P$ on the set $\Omega^{+}=\{\omega \in \Omega, \xi-1>0\}, \mathcal{F}^{-}=\Omega^{-} \cap \mathcal{F}$, $\mathcal{F}^{+}=\Omega^{+} \cap \mathcal{F}$.

It is evident that the set of measure $M_{0}$ is a nonempty one, since it contains those measures $Q$, for which the random value $\alpha\left(\omega_{1}, \omega_{2}\right)$ is bounded, since $E^{Q}|\xi-1|<\infty$. The set of measure $M_{0}$ is consistent with the filtration $\left\{\mathcal{F}_{0}, \mathcal{F}\right\}$ on the measurable space $\{\Omega, \mathcal{F}\}$, where $\mathcal{F}_{0}=\{\varnothing, \Omega\}$.

Theorem 6. On the probability space $\{\Omega, \mathcal{F}, P\}$ with the filtration $\mathcal{F}_{n}$ on it, the set of measures $M_{0}$, given by the formula (95), is consistent with the filtration $\mathcal{F}_{n}$, if and only if, as $E^{Q}\left\{\xi \mid \mathcal{F}_{n}\right\}, Q \in M_{0}$, is a local regular martingale. 
Proof. The necessity. Let the set of measures $M_{0}$ be consistent with the filtration. Then, due to Theorem 3, $E^{Q}\left\{\xi \mid \mathcal{F}_{n}\right\}, Q \in M_{0}$, is a local regular martingale.

The sufficiency. Suppose that $E^{Q}\left\{\xi \mid \mathcal{F}_{n}\right\}, Q \in M_{0}$, is a local regular martingale. Let us prove that, if $Q_{1}, Q_{2} \in M_{0}$, then the set of measures

$$
R_{s}^{k}(A)=\int_{A} \frac{E^{Q_{2}}\left\{\frac{\mathrm{d} Q_{1}}{\mathrm{~d} Q_{2}} \mid \mathcal{F}_{k}\right\}}{E^{Q_{2}}\left\{\frac{\mathrm{d} Q_{1}}{\mathrm{~d} Q_{2}} \mid \mathcal{F}_{s}\right\}} \mathrm{d} Q_{2}, A \in \mathcal{F}, k \geq s \geq n, n=\overline{0, \infty},
$$

belongs to the set $M_{0}$. For this, it is to prove that $E^{R_{s}^{k}}(\xi-1)=0$, or $E^{R_{s}^{k}} \xi=1$. Really, if $E^{Q_{1}} \xi=1, E^{Q_{2}} \xi=1$, then

$$
\begin{aligned}
E^{R_{s}^{k}} \xi= & E^{Q_{2}} \xi \frac{E^{Q_{2}}\left\{\frac{\mathrm{d} Q_{1}}{\mathrm{~d} Q_{2}} \mid \mathcal{F}_{k}\right\}}{E^{Q_{2}}\left\{\frac{\mathrm{d} Q_{1}}{\mathrm{~d} Q_{2}} \mid \mathcal{F}_{s}\right\}}=E^{Q_{2}} E^{Q_{2}}\left\{\xi \mid \mathcal{F}_{k}\right\} \frac{\frac{\mathrm{d} Q_{1}}{\mathrm{~d} Q_{2}}}{E^{Q_{2}}\left\{\frac{\mathrm{d} Q_{1}}{\mathrm{~d} Q_{2}} \mid \mathcal{F}_{s}\right\}} \\
= & E^{Q_{2}} E^{Q_{2}}\left\{E^{Q_{2}}\left\{\xi \mid \mathcal{F}_{k}\right\} \frac{\frac{\mathrm{d} Q_{1}}{\mathrm{~d} Q_{2}}}{E^{Q_{2}}\left\{\frac{\mathrm{d} Q_{1}}{\mathrm{~d} Q_{2}} \mid \mathcal{F}_{s}\right\}} \mid \mathcal{F}_{s}\right\} \\
= & E^{Q_{2}} E^{Q_{1}}\left\{E^{Q_{2}}\left\{\xi \mid \mathcal{F}_{k}\right\} \mid \mathcal{F}_{s}\right\}=E^{Q_{2}} E^{Q_{1}}\left\{E^{Q_{1}}\left\{\xi \mid \mathcal{F}_{k}\right\} \mid \mathcal{F}_{s}\right\} \\
= & E^{Q_{2}} E^{Q_{1}}\left\{\xi \mid \mathcal{F}_{s}\right\}=E^{Q_{2}} E^{Q_{2}}\left\{\xi \mid \mathcal{F}_{s}\right\}=E^{Q_{2}} \xi=1 .
\end{aligned}
$$

Theorem 6 is proved.

Theorem 7. On the probability space $\{\Omega, \mathcal{F}, P\}$ with the filtration $\mathcal{F}_{n}$ on it, the set of measures $M_{0}$, given by the formula (95), is consistent with the filtration $\mathcal{F}_{n}$, if and only if there exists not depending on $\left(\omega_{1}, \omega_{2}\right) \in \Omega^{-} \times \Omega^{+}$the random process $\left\{m_{n}, \mathcal{F}_{n}\right\}_{n=0}^{\infty}$ such that

$$
E^{v_{\omega_{1}, \omega_{2}}}\left\{\xi \mid \mathcal{F}_{n}\right\}=m_{n}, n=\overline{1, \infty}
$$

for those $\left(\omega_{1}, \omega_{2}\right) \in \Omega^{-} \times \Omega^{+}$that have the full measure $\mu=P^{-} \times P^{+}$, where

$$
\begin{aligned}
& v_{\omega_{1}, \omega_{2}}(A)=\chi_{A}\left(\omega_{1}\right) \frac{(\xi-1)^{+}\left(\omega_{2}\right)}{(\xi-1)^{-}\left(\omega_{1}\right)+(\xi-1)^{+}\left(\omega_{2}\right)} \\
& +\chi_{A}\left(\omega_{2}\right) \frac{(\xi-1)^{-}\left(\omega_{1}\right)}{(\xi-1)^{-}\left(\omega_{1}\right)+(\xi-1)^{+}\left(\omega_{2}\right)}, A \in \mathcal{F},\left(\omega_{1}, \omega_{2}\right) \in \Omega^{-} \times \Omega^{+} .
\end{aligned}
$$

Proof. The necessity. Suppose that the set of measures $M_{0}$, given by the formula (95), is consistent with the filtration $\mathcal{F}_{n}$. Due to Theorem 6, $E^{Q}\left\{\xi \mid \mathcal{F}_{n}\right\}, Q \in M_{0}$, is a local regular martingale. Then, $E^{Q}\left\{\xi \mid \mathcal{F}_{n}\right\}=m_{n}$. Using Lemma 8 , we obtain $E^{v_{\omega_{1}, \omega_{2}}}\left\{\xi \mid \mathcal{F}_{n}\right\}=m_{n}$ for those $\left(\omega_{1}, \omega_{2}\right) \in \Omega^{-} \times \Omega^{+}$that have the full measure $\mu$.

The sufficiency. If the formula (101) is true, then $E^{Q}\left\{\xi \mid \mathcal{F}_{n}\right\}=m_{n}, Q \in M_{0}$. From this, it follows that $E^{Q}\left\{\xi \mid \mathcal{F}_{n}\right\}, Q \in M_{0}$, is a local regular martingale. Theorem 7 is proved. 
Definition 5. On the probability space $\{\Omega, \mathcal{F}, P\}$ with the filtration $\mathcal{F}_{n}$ on it, the consistent with the filtration $\mathcal{F}_{n}$ subset of the measures $M$ of the set of the measures $M_{0}$ generating by the nonnegative random value $\xi \neq 1$, $E^{Q} \xi=1, Q \in M_{0}$, we call the regular set of measures.

Let $\{\Omega, \mathcal{F}, P\}$ be a probability space. On the measurable space $\{\Omega, \mathcal{F}\}$ with the filtration $\mathcal{F}_{n}$ on it, let $M \subseteq M_{0}$ be a set of regular measures, where the set $M_{0}$ is generated by the nonnegative random value $\xi \neq 1$. Denote by $\left\{m_{n}, \mathcal{F}_{n}\right\}_{n=0}^{\infty}$ the regular martingale, where $m_{n}=E^{Q}\left\{\xi \mid \mathcal{F}_{n}\right\}, Q \in M, n=\overline{1, \infty}$. Assume that $M_{n}$ is a contraction of the set of regular measures $M$ onto the $\sigma$-algebra $\mathcal{F}_{n}$. Every $Q^{n} \in M_{n}$ is equivalent to $P_{n}$, where $P_{n}$ is a contraction of the measure $P$ on the $\sigma$-algebra $\mathcal{F}_{n}$. For every $Q^{n} \in M_{n}$, we have $E^{Q^{n}}\left[m_{n}-m_{n-1}\right]=0$. Therefore, for the measure $Q^{n} \in M_{n}$ the representation

$$
\begin{gathered}
Q^{n}(A)=\int_{\Omega_{n}^{-} \times \Omega_{n}^{+}} \chi_{A}\left(\omega_{1}\right) \frac{\alpha_{n}\left(\omega_{1}, \omega_{2}\right)\left[m_{n}-m_{n-1}\right]^{+}\left(\omega_{2}\right)}{\left[m_{n}-m_{n-1}\right]^{-}\left(\omega_{1}\right)+\left[m_{n}-m_{n-1}\right]^{+}\left(\omega_{2}\right)} \mathrm{d} \mu_{n}\left(\omega_{1}, \omega_{2}\right) \\
+\int_{\Omega_{n}^{-} \times \Omega_{n}^{+}} \chi_{A}\left(\omega_{2}\right) \frac{\alpha_{n}\left(\omega_{1}, \omega_{2}\right)\left[m_{n}-m_{n-1}\right]^{-}\left(\omega_{1}\right)}{\left[m_{n}-m_{n-1}\right]^{-}\left(\omega_{1}\right)+\left[m_{n}-m_{n-1}\right]^{+}\left(\omega_{2}\right)} \mathrm{d} \mu_{n}\left(\omega_{1}, \omega_{2}\right), A \in \mathcal{F}_{n}, \\
\Omega_{n}^{-}=\left\{\omega_{1} \in \Omega,\left[m_{n}-m_{n-1}\right]\left(\omega_{1}\right) \leq 0\right\}, \\
\Omega_{n}^{+}=\left\{\omega_{2} \in \Omega,\left[m_{n}-m_{n-1}\right]\left(\omega_{2}\right)>0\right\},
\end{gathered}
$$

is true, where, on the measurable space $\left\{\Omega_{n}^{-} \times \Omega_{n}^{+}, \mathcal{F}_{n}^{-} \times \mathcal{F}_{n}^{+}\right\}$, the random value $\alpha_{n}\left(\omega_{1}, \omega_{2}\right)$ satisfies the conditions

$$
\begin{gathered}
\mu_{n}\left(\left\{\left(\omega_{1}, \omega_{2}\right) \in \Omega_{n}^{-} \times \Omega_{n}^{+}, \alpha_{n}\left(\omega_{1}, \omega_{2}\right)>0\right\}\right)=P_{n}\left(\Omega^{+}\right) P_{n}\left(\Omega^{-}\right), \\
\iint_{\Omega_{n}^{-} \Omega_{n}^{+}} \alpha_{n}\left(\omega_{1}, \omega_{2}\right) \frac{\left[m_{n}-m_{n-1}\right]^{-}\left(\omega_{1}\right)\left[m_{n}-m_{n-1}\right]^{+}\left(\omega_{2}\right)}{\left[m_{n}-m_{n-1}\right]^{-}\left(\omega_{1}\right)+\left[m_{n}-m_{n-1}\right]^{+}\left(\omega_{2}\right)} \mathrm{d} \mu_{n}\left(\omega_{1}, \omega_{2}\right)<\infty, \\
\iint_{\Omega_{n}^{-} \Omega_{n}^{+}} \alpha_{n}\left(\omega_{1}, \omega_{2}\right) \mathrm{d} \mu_{n}\left(\omega_{1}, \omega_{2}\right)=1 .
\end{gathered}
$$

Here, the measure $\mu_{n}=P_{n}^{-} \times P_{n}^{+}$is given on the measurable space $\left\{\Omega_{n}^{-} \times \Omega_{n}^{+}, \mathcal{F}_{n}^{-} \times \mathcal{F}_{n}^{+}\right\}$and it is a direct product of the measures $P_{n}^{-}$and $P_{n}^{+}$, where the measure $P_{n}^{+}$is a contraction of the measure $P_{n}$ on the $\sigma$-algebra $\mathcal{F}_{n}^{+}=\Omega_{n}^{+} \cap \mathcal{F}_{n}$ and $P_{n}^{-}$is a contraction of the measure $P_{n}$ on the $\sigma$-algebra $\mathcal{F}_{n}^{-}=\Omega_{n}^{-} \cap \mathcal{F}_{n}$. It is evident that the regular set of measures $M$ is a convex set of measure.

Definition 6. On the probability space $\{\Omega, \mathcal{F}, P\}$ with the filtration $\mathcal{F}_{n}$ on $i$, denote by $A_{0}$ the set of all nonnegative integrable random values $\zeta$ relative to the set of regular measures $M$, satisfying the conditions.

$$
E^{P} \zeta=1, P \in M \text {. }
$$

Due to Theorem 3, $\left\{E^{P}\left\{\zeta \mid \mathcal{F}_{n}\right\}, \mathcal{F}_{n}\right\}_{n=0}^{\infty}$ is a regular martingale relative to the set of measures $M$.

It is evident that the set $A_{0}$ is a nonempty one, since it contains the random value $\zeta=1$. The more interesting case is as $A_{0}$ contains more than one ele- 
ment. So, further we consider the regular set of measure $M$ with the set $A_{0}$, containing more than one element.

In the next Lemma 9, using Lemma 5, we construct a set of measures consistent with the filtration. On the probability space $\left\{\Omega_{1}^{0}, \mathcal{F}_{1}^{0}, P_{1}^{0}\right\}$, let us consider a nonnegative random value $\xi_{1}$, satisfying the conditions

$$
\begin{gathered}
0<P_{1}^{0}\left(\left\{\omega_{1} \in \Omega_{1}^{0}, \eta_{1}\left(\omega_{1}\right)<0\right\}\right)<1, \\
0<P_{1}^{0}\left(\left\{\omega_{1} \in \Omega_{1}^{0}, \eta_{1}\left(\omega_{1}\right)>0\right\}\right),
\end{gathered}
$$

where we introduced the denotation $\eta_{1}\left(\omega_{1}\right)=\xi_{1}\left(\omega_{1}\right)-1$. Described in Lemma 5 the set of equivalent measures to the measure $P_{1}^{0}$ and such that $E^{Q} \eta_{1}\left(\omega_{1}\right)=0$, we denote by $M_{1}$. Let us construct the infinite direct product of the measurable spaces $\left\{\Omega_{i}^{0}, \mathcal{F}_{i}^{0}\right\}, i=\overline{1, \infty}$, where $\Omega_{i}^{0}=\Omega_{1}^{0}, \mathcal{F}_{i}^{0}=\mathcal{F}_{1}^{0}$. Denote $\Omega=\prod_{i=1}^{\infty} \Omega_{i}^{0}$. On the space $\Omega$, under the $\sigma$-algebra $\mathcal{F}$ we understand the minimal $\sigma$-algebra, generated by the sets $\prod_{i=1}^{\infty} G_{i}, G_{i} \in F_{i}^{0}$, where in the last product only the finite set of $G_{i}$ do not equal $\Omega_{i}^{0}$. On the measurable space $\{\Omega, \mathcal{F}\}$, under the filtration $\mathcal{F}_{n}$ we understand the minimal $\sigma$-algebra generated by the sets $\prod_{i=1}^{\infty} G_{i}, G_{i} \in \mathcal{F}_{i}^{0}$, where $G_{i}=\Omega_{i}^{0}$ for $i>n$. We consider the probability space $\{\Omega, \mathcal{F}, P\}$, where $P=\prod_{i=1}^{\infty} P_{i}^{0}, P_{i}^{0}=P_{1}^{0}, i=\overline{1, \infty}$.

On the measurable space $\{\Omega, \mathcal{F}\}$, we introduce into consideration the set of measures $M$, where $Q$ belongs to $M$, if $Q=\prod_{i=1}^{\infty} Q_{i}, Q_{i} \in M_{1}$. We denote by $M^{Q_{0}}$ a subset of the set $M$ of those measures $Q=\prod_{i=1}^{\infty} Q_{i}, Q_{i} \in M_{1}$, for which only the finite set of $Q_{i}$ does not coincide with the measure $Q_{0} \in M_{1}$.

Lemma 9. On the measurable space $\{\Omega, \mathcal{F}\}$ with the filtration $\mathcal{F}_{n}$ on it, there exists consistent with the filtration $\mathcal{F}_{n}$ the set of measures $M_{0}$ and the nonnegative random variable $\xi_{0}$ such that $E^{Q} \xi_{0}=1, Q \in M_{0}$, if the random value $\xi_{1}$, satisfying the conditions (108), is bounded.

Proof. To prove Lemma 9, we need to construct a nonnegative bounded random value $\xi_{0}$ on the measurable space $\{\Omega, \mathcal{F}\}$ and a set of equivalent measures $M_{0}$ on it, such that $E^{Q} \xi_{0}=1, Q \in M_{0}$, and to prove that the set of measures $M_{0}$ is consistent with the filtration $\mathcal{F}_{n}$. From the Lemma 9 conditions, the random value $\eta_{1}\left(\omega_{1}\right)=\xi_{1}\left(\omega_{1}\right)-1$ is also bounded. Let us put

$$
\xi_{0}=\prod_{i=1}^{\infty}\left[1+a_{i}\left(\omega_{1}, \cdots, \omega_{i-1}\right) \eta_{i}\left(\omega_{i}\right)\right] \text {, }
$$

where the random values $a_{i}\left(\omega_{1}, \cdots, \omega_{i-1}\right)$ are $\mathcal{F}_{i-1}$-measurable, $i=\overline{1, \infty}$, they satisfy the conditions $0<a_{i}\left(\omega_{1}, \cdots, \omega_{i-1}\right) \leq b_{i}<1$. The constants $b_{i}$ are such that $\sum_{i=1}^{\infty} b_{i}<\infty$, the random value $\eta_{i}\left(\omega_{i}\right)$ is given on $\left\{\Omega_{i}^{0}, \mathcal{F}_{i}^{0}, P_{i}^{0}\right\}$ and is 
distributed as $\eta_{1}\left(\omega_{1}\right)$ on $\left\{\Omega_{1}^{0}, \mathcal{F}_{1}^{0}, P_{1}^{0}\right\}$. From this, it follows that the random value $\xi_{0}$ is bounded by the constant $\prod_{i=1}^{\infty}\left[1+C b_{i}\right]$, where $C>0$ and it is such that $\left|\eta_{i}\left(\omega_{i}\right)\right|<C, i=\overline{1, \infty}$. It is evident that $E^{Q} \xi_{0}=1, Q \in M^{Q_{0}}$. Really,

$$
\begin{aligned}
& E^{Q} \prod_{i=1}^{n}\left[1+a_{i}\left(\omega_{1}, \cdots, \omega_{i-1}\right) \eta_{i}\left(\omega_{i}\right)\right] \\
& =E^{Q_{0}^{n-1}} \prod_{i=1}^{n-1}\left[1+a_{i}\left(\omega_{1}, \cdots, \omega_{i-1}\right) \eta_{i}\left(\omega_{i}\right)\right] \\
& \times E^{Q_{n}}\left[1+a_{n-1}\left(\omega_{1}, \cdots, \omega_{i-1}\right) \eta_{n}\left(\omega_{n}\right)\right],
\end{aligned}
$$

where $Q=\prod_{i=1}^{\infty} Q_{i}, Q_{0}^{n-1}=\prod_{i=1}^{n-1} Q_{i}$,

$$
\begin{aligned}
& E^{Q_{n}}\left[1+a_{n-1}\left(\omega_{1}, \cdots, \omega_{n-1}\right) \eta_{i}\left(\omega_{n}\right)\right] \\
& =\left[1+a_{n-1}\left(\omega_{1}, \cdots, \omega_{n-1}\right) E^{Q_{n}} \eta_{n}\left(\omega_{n}\right)\right]=1 .
\end{aligned}
$$

From the last equality, we have

$$
E^{Q} \prod_{i=1}^{n}\left[1+a_{i}\left(\omega_{1}, \cdots, \omega_{i-1}\right) \eta_{i}\left(\omega_{i}\right)\right]=1 .
$$

Since $\xi_{0}=\lim _{n \rightarrow \infty} \prod_{i=1}^{n}\left[1+a_{i}\left(\omega_{1}, \cdots, \omega_{i-1}\right) \eta_{i}\left(\omega_{i}\right)\right]$, from the equality (112) and the possibility to go to the limit under the mathematical expectation, we prove the needed statement. Let us prove the existence of the set of measures $M_{0}$ consistent with the filtration $\mathcal{F}_{n}$. If $Q \in M^{Q_{0}}$, then

$$
E^{Q}\left\{\xi_{0} \mid \mathcal{F}_{n}\right\}=\prod_{i=1}^{n}\left[1+a_{i}\left(\omega_{1}, \cdots, \omega_{i-1}\right) \eta_{i}\left(\omega_{i}\right)\right], Q \in M^{Q_{0}}
$$

Due to Lemma 4, there exists a set of measures $M_{0}$ such that it is consistent with the filtration and $M_{0} \supseteq M^{Q_{0}}, E^{Q} \xi_{0}=1, Q \in M_{0}$. The set $M_{0}$ is a linear convex span of the set $M^{Q_{0}}$. It means that the set of measures $M_{0}$ is consistent with the filtration. Lemma 9 is proved.

Remark 2 The boundedness of the random value $\xi_{1}$ is not essential. For the applications, the case, as $a_{i}\left(\omega_{1}, \cdots, \omega_{i-1}\right)=0, i \geq n+1$, is very important (see Section 8). In this case, Lemma 9 is true as the random value $\eta_{1}$ is an integrable one. The random value $\xi_{0}$ is also integrable one relative to every measures from the set $M_{0}$ and it is $\mathcal{F}_{n}$-measurable one.

Below, we describe one class of evolutions of risk assets satisfying no arbitrage condition [15]-[20] and give the complete description of the set of equivalent martingale measures.

On the introduced measurable space $\{\Omega, \mathcal{F}, P\}$ we consider the evolution of the risk asset given by the law

$$
S_{n}=S_{n-1}\left(1+a_{n}\left(\omega_{1}, \cdots, \omega_{n-1}\right)\right) \eta_{n}\left(\omega_{n}\right), n=\overline{1, N},
$$

where the random values $a_{i}\left(\omega_{1}, \cdots, \omega_{i-1}\right)$ are $\mathcal{F}_{i-1}$-measurable, $i=\overline{1, N}$, they satisfy the conditions $0<a_{i}\left(\omega_{1}, \cdots, \omega_{i-1}\right) \leq 1, \eta_{1}\left(\omega_{1}\right)=\xi_{1}\left(\omega_{1}\right)-1$, the random 
value $\eta_{i}\left(\omega_{i}\right)$ is given on $\left\{\Omega_{i}^{0}, \mathcal{F}_{i}^{0}, P_{i}^{0}\right\}$ and is distributed as $\eta_{1}\left(\omega_{1}\right)$ on $\left\{\Omega_{1}^{0}, \mathcal{F}_{1}^{0}, P_{1}^{0}\right\}$. The main aim is to describe the set of martingale measures for the evolution of risk asset given by the formula (114). This problem we solve in Theorem 8.

Below, we describe completely the regular set of measures in the case as $\xi_{0}=\prod_{i=1}^{N}\left[1+a_{i}\left(\omega_{1}, \cdots, \omega_{i-1}\right) \eta_{i}\left(\omega_{i}\right)\right], N<\infty, \quad 0<a_{i}\left(\omega_{1}, \cdots, \omega_{i-1}\right) \leq 1, i=\overline{1, N}$, and the random value $\xi_{1}$ is an integrable one relative to the measure $P_{1}^{0}$. For this purpose, we introduce the denotations: $\Omega_{1}^{-}=\left\{\omega_{1} \in \Omega_{1}^{0}, \eta_{1}\left(\omega_{1}\right) \leq 0\right\}$, $\Omega_{1}^{+}=\left\{\omega_{1} \in \Omega_{1}^{0}, \eta_{1}\left(\omega_{1}\right)>0\right\}, P_{1}^{-}$is a contraction of the measure $P_{1}^{0}$ on the $\sigma$-algebra $\mathcal{F}_{1}^{-}, P_{1}^{+}$is a contraction of the measure $P_{1}^{0}$ on the $\sigma$-algebra $\mathcal{F}_{1}^{+}$, $\mathcal{F}_{1}^{-}=\Omega_{1}^{-} \cap \mathcal{F}_{1}^{0}, \mathcal{F}_{1}^{+}=\Omega_{1}^{+} \cap \mathcal{F}_{1}^{0}$.

Denote $U_{1}=\Omega_{1}^{-} \times \Omega_{1}^{+}$and introduce the measure $\mu_{1}=P_{1}^{-} \times P_{1}^{+}$on the $\sigma$-algebra $G_{1}=\mathcal{F}_{1}^{-} \times \mathcal{F}_{1}^{+}$. Let us introduce the measurable space $\{\mathcal{V}, \mathcal{L}, \mu\}$, where $\mathcal{V}=\prod_{i=1}^{N} U_{i}, U_{i}=U_{1}, i=\overline{1, N}$, is a direct product of the spaces $U_{i}=\Omega_{i}^{-} \times \Omega_{i}^{+}, \quad \Omega_{i}^{-}=\Omega_{1}^{-}, \quad \Omega_{i}^{+}=\Omega_{1}^{+}, \quad \mathcal{L}=\prod_{i=1}^{N} G_{i}$ is a direct product of the $\sigma$-algebras $G_{i}=G_{1}, i=\overline{1, N}$. At last, let $\mu=\prod_{i=1}^{N} \mu_{i}$ be a direct product of the measures $\mu_{i}=\mu_{1}, i=\overline{1, N}$, and let $v_{v}=\prod_{i=1}^{N} v_{\omega_{i}^{1}, \omega_{i}^{2}}, v=\left\{\left(\omega_{1}^{1}, \omega_{1}^{2}\right), \cdots,\left(\omega_{N}^{1}, \omega_{N}^{2}\right)\right\}$, be a direct product of the measures $v_{\omega_{i}^{1}, \omega_{i}^{2}}, i=\overline{1, N}$, which is a countable additive function on the $\sigma$-algebra $\mathcal{F}_{N}$ for every $v \in \mathcal{V}$, where

$$
v_{\omega_{i}^{1}, \omega_{i}^{2}}\left(A_{i}\right)=\chi_{A_{i}}\left(\omega_{i}^{1}\right) \frac{\eta_{i}^{+}\left(\omega_{i}^{2}\right)}{\eta_{i}^{-}\left(\omega_{i}^{1}\right)+\eta_{i}^{+}\left(\omega_{i}^{2}\right)}+\chi_{A_{i}}\left(\omega_{i}^{2}\right) \frac{\eta_{i}^{-}\left(\omega_{i}^{1}\right)}{\eta_{i}^{-}\left(\omega_{i}^{1}\right)+\eta_{i}^{+}\left(\omega_{i}^{2}\right)}
$$

for $\omega_{i}^{1} \in \Omega_{i}^{-}, \omega_{i}^{2} \in \Omega_{i}^{+}, A_{i} \in \mathcal{F}_{i}^{0}$.

In the next Theorem 8 , we assume that the random value $\eta_{1}\left(\omega_{1}\right)$ is an integrable one.

Theorem 8. On the measurable space $\{\Omega, \mathcal{F}\}$ with the filtration $\mathcal{F}_{n}$ on it, every measure $Q$ of the regular set of measures $M$ for the random value $\xi_{0}=\prod_{i=1}^{N}\left[1+a_{i}\left(\omega_{1}, \cdots, \omega_{i-1}\right) \eta_{i}\left(\omega_{i}\right)\right], N<\infty, \quad 0<a_{i}\left(\omega_{1}, \cdots, \omega_{i-1}\right) \leq 1, i=\overline{1, N}$, has the representation

$$
Q(A)=\int_{V} \alpha(v) v_{v}(A) \mathrm{d} \mu(v),
$$

where the random value $\alpha(v)$ satisfies the conditions

$$
\begin{gathered}
\mu(\{v \in \mathcal{V}, \alpha(v)>0\})=\left[P_{1}^{0}\left(\Omega_{1}^{-}\right) P_{1}^{0}\left(\Omega_{1}^{+}\right)\right]^{N}, \\
\int_{\mathcal{V}} \alpha(v) \prod_{i=1}^{N} \frac{\eta_{i}^{-}\left(\omega_{i}^{1}\right) \eta_{i}^{+}\left(\omega_{i}^{2}\right)}{\eta_{i}^{-}\left(\omega_{i}^{1}\right)+\eta_{i}^{+}\left(\omega_{i}^{2}\right)} \mathrm{d} \mu(v)<\infty, \\
\int_{\mathcal{V}} \alpha(v) \mathrm{d} \mu(v)=1 .
\end{gathered}
$$


Proof. To prove Theorem, it needs to prove that the countable additive measure $v_{v}(A)$ at every fixed $v \in \mathcal{V}$ is a measurable map from the measurable space $\{\mathcal{V}, \mathcal{L}\}$ into the measurable space $\{[0,1], B([0,1])\}$ for every fixed $A \in \mathcal{F}_{N}$. For $A=\prod_{i=1}^{N} A_{i}, A_{i} \in \mathcal{F}_{i}^{0}, v_{v}(A)$ is a measurable map from the measurable space $\{\mathcal{V}, \mathcal{L}\}$ into the measurable space $\{[0,1], B([0,1])\}$. The family of sets of the kind $\bigcup_{i \in I} E_{i}, \quad E_{i}=\prod_{s=1}^{N} A_{s}^{i}, A_{s}^{i} \in F_{s}^{0}$, where $E_{i} \cap E_{j}=\varnothing$, the set $I$ is an arbitrary finite set, forms the algebra of the sets that we denote by $U_{0}$. From the countable additivity of $v_{v}(A), v_{v}\left(\bigcup_{i \in I} E_{i}\right)=\sum_{i \in I} v_{v}\left(E_{i}\right)$ is a measurable map from the measurable space $\{\mathcal{V}, \mathcal{L}\}$ into the measurable space $\{[0,1], B([0,1])\}$. Let $T$ be a class of the sets from the minimal $\sigma$-algebra $\Sigma$ generated by $U_{0}$ for every subset $E$ of that $v_{v}(E)$ is a measurable map from the measurable space $\{\mathcal{V}, \mathcal{L}\}$ into the measurable space $\{[0,1], B([0,1])\}$. Let us prove that $T$ is a monotonic class. Suppose that $E_{i} \subset E_{i+1}, i=\overline{1, \infty}, E_{i} \in T$. Then,

$v_{v}\left(E_{i}\right) \leq v_{v}\left(E_{i+1}\right)$. From this, it follows that $\lim _{i \rightarrow \infty} v_{v}\left(E_{i}\right)$ is a measurable map from the measurable space $\{\mathcal{V}, \mathcal{L}\}$ into the measurable space $\{[0,1], B([0,1])\}$. But, $v_{v}\left(E_{i+1} \backslash E_{i}\right)=v_{v}\left(E_{i+1}\right)-v_{v}\left(E_{i}\right)$ is a measurable map from $\{\mathcal{V}, \mathcal{L}\}$ into $\{[0,1], B([0,1])\}$. From this equality, it follows that the set $E_{i+1} \backslash E_{i}$ belongs to the class $T$. Since $\bigcup_{i=1}^{\infty} E_{i}=E_{1} \cup \bigcup_{i=1}^{\infty}\left[E_{i+1} \backslash E_{i}\right]$, we have

$$
\begin{aligned}
\lim _{n \rightarrow \infty} v_{v}\left(E_{n}\right) & =v_{v}\left(E_{1}\right)+\lim _{n \rightarrow \infty} \sum_{i=1}^{n} v_{v}\left(E_{i+1} \backslash E_{i}\right) \\
& =v_{v}\left(E_{1}\right)+\sum_{i=1}^{\infty} v_{v}\left(E_{i+1} \backslash E_{i}\right) \\
& =v_{v}\left(E_{1} \bigcup \bigcup_{i=1}^{\infty}\left[E_{i+1} \backslash E_{i}\right]\right)=v_{v}\left(\bigcup_{i=1}^{\infty} E_{i}\right) .
\end{aligned}
$$

The equalities (120) mean that $\bigcup_{i=1}^{\infty} E_{i}$ belongs to $T$, since $v_{v}\left(\bigcup_{i=1}^{\infty} E_{i}\right)$ is a measurable map of $\{\mathcal{V}, \mathcal{L}\}$ into $\{[0,1], B([0,1])\}$. Suppose that $E_{i} \supset E_{i+1}, E_{i} \in T, i=\overline{1, \infty}$. Then, this case is reduced to the previous one by the note that the sequence $\bar{E}_{i}=\prod_{i=1}^{N} \Omega_{i}^{0} \backslash E_{i}, i=\overline{1, \infty}$ is monotonically increasing. From this, it follows that $\bar{E}=\bigcup_{i=1}^{\infty} \bar{E}_{i} \in T$. Therefore, $\bigcap_{i=1}^{\infty} E_{i}=\prod_{i=1}^{N} \Omega_{i}^{0} \backslash \bigcup_{i=1}^{\infty} \bar{E}_{i} \in T$. Thus, $T$ is a monotone class. But, $U_{0} \subset T$. Hence, $T$ contains the minimal monotone class generated by the algebra $U_{0}$, that is, $m\left(U_{0}\right)=\Sigma$, therefore, $\Sigma \subset T$. Thus, $v_{v}(E)$ is a measurable map of $\{\mathcal{V}, \mathcal{L}\}$ into $\{[0,1], B([0,1])\}$ for $A \in \Sigma$. The fact that the random value $\alpha(v)$ satisfies the conditions (117)-(119) means that $Q$, given by the formula (116), is a countable additive function of sets and $E^{Q} \xi_{0}<\infty$. Moreover, $E^{Q} \xi_{0}=1$. It is evident that 
$E^{Q}\left\{\xi_{0} \mid \mathcal{F}_{n}\right\}=\prod_{i=1}^{n}\left[1+a_{i}\left(\omega_{1}, \cdots, \omega_{i-1}\right) \eta_{i}\left(\omega_{i}\right)\right], Q \in M$. Due to Lemma 4 , this proves that the set $M$ is a regular set of measure. Theorem 8 is proved.

Remark 3. The representation (116) for the regular set of measures $M$ means that $M$ is a convex set of equivalent measures. Since the random value $\alpha(v)$ runs all bounded random values, satisfying the conditions (117 - 119), it is easy to show that the set of measures $v_{v}(A), v \in \mathcal{V}, A \in \mathcal{F}_{N}$, is the set of extreme points for the set $M$.

Let us introduce the denotations

$$
\begin{aligned}
& m_{n}(\{\omega\})=\prod_{i=1}^{n}\left[1+a_{i}\left(\omega_{1}, \cdots, \omega_{i-1}\right) \eta_{i}\left(\omega_{i}\right)\right], 1 \leq n \leq N<\infty \\
& 0<a_{i}\left(\omega_{1}, \cdots, \omega_{i-1}\right) \leq 1, i=\overline{1, N} . \\
& \{\omega\}=\left\{\omega_{1}, \cdots, \omega_{N}\right\},\{\omega\}^{i}=\left\{\omega_{1}^{i}, \cdots, \omega_{N}^{i}\right\}, i=1,2, \Omega_{n}=\prod_{i=1}^{n} \Omega_{i}^{0}, n=\overline{1, N}, \\
& \{\omega\}_{n}^{1}=\left\{\omega_{1}^{1}, \cdots, \omega_{n}^{1}\right\},\{\omega\}_{n}^{i}=\left\{\omega_{1}^{i}, \cdots, \omega_{n}^{i}\right\}, i=1,2, \\
& \Omega_{N-n}=\prod_{i=n+1}^{N} \Omega_{i}^{0}, n=\overline{1, N-1}, \\
& \bar{\Omega}_{n}^{-}=\left\{\{\omega\}_{n} \in \Omega_{n},\left[m_{n}-m_{n-1}\right]\left(\{\omega\}_{n}\right) \leq 0\right\} \\
& =\Omega_{n-1} \times\left\{\omega_{n} \in \Omega_{n}^{0}, \eta_{n}\left(\omega_{n}\right) \leq 0\right\}, \\
& \bar{\Omega}_{n}^{+}=\left\{\{\omega\}_{n} \in \Omega_{n},\left[m_{n}-m_{n-1}\right]\left(\{\omega\}_{n}\right)>0\right\} \\
& =\Omega_{n-1} \times\left\{\omega_{n} \in \Omega_{n}^{0}, \eta_{n}\left(\omega_{n}\right)>0\right\}, \\
& \Omega_{n}^{-}=\left\{\{\omega\} \in \Omega_{N},\left[m_{n}-m_{n-1}\right](\{\omega\}) \leq 0\right\} \\
& =\Omega_{n-1} \times\left\{\omega_{n} \in \Omega_{n}^{0}, \eta_{n}\left(\omega_{n}\right) \leq 0\right\} \times \Omega_{N-n}, \\
& \Omega_{n}^{+}=\left\{\{\omega\} \in \Omega_{N},\left[m_{n}-m_{n-1}\right](\{\omega\})>0\right\} \\
& =\Omega_{n-1} \times\left\{\omega_{n} \in \Omega_{n}^{0}, \eta_{n}\left(\omega_{n}\right)>0\right\} \times \Omega_{N-n} .
\end{aligned}
$$

Note that the $\sigma$-algebra $\mathcal{F}_{n}$ is generated by sets of the kind $G=\prod_{i=1}^{N} G_{i}$, where $G_{i} \in \mathcal{F}_{i}^{0}, i=\overline{1, n}, G_{i}=\Omega_{i}^{0}, i=\overline{n+1, N}$. Denote $P_{n}=\prod_{i=1}^{n} P_{i}^{0}$ the contraction of the measure $P_{N}=\prod_{i=1}^{N} P_{i}^{0}$ onto the $\sigma$-algebra $\mathcal{F}_{n}$. Further we use the denotations $P_{n}^{-}$and $P_{n}^{+}$which are the contractions the measure $P_{n}$ onto the $\sigma$-algebras $\mathcal{F}_{n} \cap \Omega_{n}^{-}$and $\mathcal{F}_{n} \cap \Omega_{n}^{+}$, correspondingly. If the measure $Q$ belongs to the set of martingale measures (116), then $E^{Q}\left\{m_{n} \mid \mathcal{F}_{n-1}\right\}=m_{n-1}$, or $E^{Q}\left[m_{n}-m_{n-1}\right]=0$. From this, for the measure $Q$ the representation 


$$
\begin{aligned}
& Q\left(A_{1}\right)=\int_{\Omega_{n}^{-} \times \Omega_{n}^{+}} \frac{\chi_{A_{1}}\left(\{\omega\}^{1}\right) \alpha\left(\{\omega\}^{1} ;\{\omega\}^{2}\right)\left[m_{n}-m_{n-1}\right]^{+}\left(\{\omega\}^{2}\right)}{\left[m_{n}-m_{n-1}\right]^{-}\left(\{\omega\}^{1}\right)+\left[m_{n}-m_{n-1}\right]^{+}\left(\{\omega\}^{2}\right)} \mathrm{d}\left[P_{N}^{-} \times P_{N}^{+}\right] \\
& +\int_{\Omega_{n}^{-} \times \Omega_{n}^{+}} \frac{\chi_{A_{1}}\left(\{\omega\}^{2}\right) \alpha\left(\{\omega\}^{1} ;\{\omega\}^{2}\right)\left[m_{n}-m_{n-1}\right]^{-}\left(\{\omega\}^{1}\right)}{\left[m_{n}-m_{n-1}\right]^{-}\left(\{\omega\}^{1}\right)+\left[m_{n}-m_{n-1}\right]^{+}\left(\{\omega\}^{2}\right)} \mathrm{d}\left[P_{N}^{-} \times P_{N}^{+}\right], A_{1} \in \mathcal{F}_{n},
\end{aligned}
$$

is true if the random value $\alpha\left(\{\omega\}^{1} ;\{\omega\}^{2}\right)>0$ satisfies the condition

$$
\int_{\Omega_{n}^{-} \times \Omega_{n}^{+}} \alpha\left(\{\omega\}^{1} ;\{\omega\}^{2}\right) \mathrm{d}\left[P_{N}^{-} \times P_{N}^{+}\right]=1 .
$$

Since for the set $A_{1}$ the representation $A_{1}=A \times \prod_{i=n+1}^{N} \Omega_{i}^{0}$, is true, where $A \in \overline{\mathcal{F}}_{n}=\prod_{i=1}^{n} \mathcal{F}_{i}^{0}$, then for the contraction $Q_{n}$ of the measure $Q$ onto the $\sigma$-algebra $\overline{\mathcal{F}}_{n}$ the representation

$$
\begin{aligned}
& Q_{n}(A)=\int_{\bar{\Omega}_{n}^{-} \times \bar{\Omega}_{n}^{+}} \frac{\chi_{A}\left(\{\omega\}_{n}^{1}\right) \alpha_{n}^{1}\left(\{\omega\}_{n}^{1} ;\{\omega\}_{n}^{2}\right)\left[m_{n}-m_{n-1}\right]^{+}\left(\{\omega\}_{n}^{2}\right)}{\left[m_{n}-m_{n-1}\right]^{-}\left(\{\omega\}_{n}^{1}\right)+\left[m_{n}-m_{n-1}\right]^{+}\left(\{\omega\}_{n}^{2}\right)} \mathrm{d}\left[\bar{P}_{n}^{-} \times \bar{P}_{n}^{+}\right] \\
& +\int_{\bar{\Omega}_{n}^{\times} \times \bar{\Omega}_{n}^{+}} \frac{\chi_{A}\left(\{\omega\}_{n}^{2}\right) \alpha_{n}^{1}\left(\{\omega\}_{n}^{1} ;\{\omega\}_{n}^{2}\right)\left[m_{n}-m_{n-1}\right]^{-}\left(\{\omega\}_{n}^{1}\right)}{\left[m_{n}-m_{n-1}\right]^{-}\left(\{\omega\}_{n}^{1}\right)+\left[m_{n}-m_{n-1}\right]^{+}\left(\{\omega\}_{n}^{2}\right)} \mathrm{d}\left[\bar{P}_{n}^{-} \times \bar{P}_{n}^{+}\right], \quad A \in \overline{\mathcal{F}}_{n},
\end{aligned}
$$

is true, where we introduced the denotations $\bar{P}_{n}^{-}$and $\bar{P}_{n}^{+}$which are the contractions of the measure $P_{n}$ onto the $\sigma$-algebras $\overline{\mathcal{F}}_{n} \cap \bar{\Omega}_{n}^{-}$and $\overline{\mathcal{F}}_{n} \cap \bar{\Omega}_{n}^{+}$, correspondingly,

$$
\begin{gathered}
\alpha_{n}^{1}\left(\{\omega\}_{n}^{1} ;\{\omega\}_{n}^{2}\right)=\int_{\Omega_{N-n} \times \Omega_{N-n}} \alpha\left(\{\omega\}^{1} ;\{\omega\}^{2}\right) \mathrm{d}\left[P_{N-n} \times P_{N-n}\right], \\
P_{N-n}=\prod_{i=n+1}^{N} P_{i}^{0}, \int_{\Omega_{n}^{-} \times \bar{\Omega}_{n}^{+}} \alpha_{n}^{1}\left(\{\omega\}_{n}^{1} ;\{\omega\}_{n}^{2}\right) \mathrm{d}\left[\bar{P}_{n}^{-} \times \bar{P}_{n}^{+}\right]=1 .
\end{gathered}
$$

In the set $\bar{\Omega}_{n}^{-} \times \bar{\Omega}_{n}^{+}$let us introduce the transformation

$$
\begin{gathered}
T_{n}\left(\{\omega\}_{n}^{1} ;\{\omega\}_{n}^{2}\right)=\left(T_{n}^{1}\left(\{\omega\}_{n}^{1}\right) ; T_{n}^{2}\left(\{\omega\}_{n}^{2}\right)\right), \\
T_{n}^{1}\left(\{\omega\}_{n}^{1}\right)=\left\{\{\omega\}_{n-1}^{2}, \omega_{n}^{1}\right\}, T_{n}^{2}\left(\{\omega\}_{n}^{2}\right)=\left\{\{\omega\}_{n-1}^{1}, \omega_{n}^{2}\right\}, n=\overline{1, N} .
\end{gathered}
$$

By the definition we put that for $n=1$ the transformation $T_{1}$ is identical one. Introduce the denotations

$$
\begin{gathered}
v_{n}^{1}\left(\{\omega\}_{n}^{1} ;\{\omega\}_{n}^{2}\right)=\frac{\left[m_{n}-m_{n-1}\right]^{+}\left(\{\omega\}_{n}^{2}\right)}{\varphi_{n}\left(\{\omega\}_{n}^{1} ;\{\omega\}_{n}^{2}\right)}, \\
v_{n}^{2}\left(\{\omega\}_{n}^{1} ;\{\omega\}_{n}^{2}\right)=\frac{\left[m_{n}-m_{n-1}\right]^{-}\left(\{\omega\}_{n}^{1}\right)}{\varphi_{n}\left(\{\omega\}_{n}^{1} ;\{\omega\}_{n}^{2}\right)} . \\
\varphi_{n}^{1}\left(\{\omega\}_{n}^{1} ;\{\omega\}_{n}^{2}\right)=\left[m_{n}-m_{n-1}\right]^{-}\left(\{\omega\}_{n}^{1}\right)+\left[m_{n}-m_{n-1}\right]^{+}\left(\{\omega\}_{n}^{2}\right),
\end{gathered}
$$




$$
\varphi_{n}\left(\{\omega\}_{n}^{1} ;\{\omega\}_{n}^{2}\right)=\varphi_{n}^{1}\left(\{\omega\}_{n}^{1} ;\{\omega\}_{n}^{2}\right)+\varphi_{n}^{1}\left(T_{n}\left(\{\omega\}_{n}^{1} ;\{\omega\}_{n}^{2}\right)\right) .
$$

Theorem 9. Let $\Omega_{1}^{0}$ be a complete separable metric space and $\mathcal{F}_{1}^{0}$ be a Borel $\sigma$-algebra on it. If the condition

$$
\int_{\Omega_{n}} f\left(\{\omega\}_{n}\right) \mathrm{d} P_{n}<\infty,
$$

is true for $\mathcal{F}_{n}$-measurable nonnegative random value $f\left(\{\omega\}_{n}\right)$, then the closure of the set of points $E^{Q_{n}} f\left(\{\omega\}_{n}\right), Q_{n} \in M_{n}$, in metrics $\rho(x, y)=|x-y|$ on the real line contains the set of points

$$
\begin{aligned}
& f\left(\{\omega\}_{n}^{1}\right) v_{n}^{1}\left(\{\omega\}_{n}^{1} ;\{\omega\}_{n}^{2}\right)+f\left(\{\omega\}_{n}^{2}\right) v_{n}^{2}\left(\{\omega\}_{n}^{1} ;\{\omega\}_{n}^{2}\right) \\
& +f\left(T_{n}^{1}\left(\{\omega\}_{n}^{1}\right)\right) v_{n}^{1}\left(T_{n}\left(\{\omega\}_{n}^{1} ;\{\omega\}_{n}^{2}\right)\right) \\
& +f\left(T_{n}^{2}\left(\{\omega\}_{n}^{2}\right)\right) v_{n}^{2}\left(T_{n}\left(\{\omega\}_{n}^{1} ;\{\omega\}_{n}^{2}\right)\right), n=\overline{1, N} .
\end{aligned}
$$

Proof. Let us find the conditions for the measurable functions $\alpha_{n}^{1}\left(\{\omega\}_{n}^{1} ;\{\omega\}_{n}^{2}\right)$ under which $E^{Q_{n}}\left\{m_{n} \mid \overline{\mathcal{F}}_{n-1}\right\}=m_{n-1}$. Introduce the denotation

$$
\alpha_{n}^{0}\left(\{\omega\}_{n}^{1} ;\{\omega\}_{n}^{2}\right)=\frac{\alpha_{n}^{1}\left(\{\omega\}_{n}^{1} ;\{\omega\}_{n}^{2}\right)}{\left[m_{n}-m_{n-1}\right]^{-}\left(\{\omega\}_{n}^{1}\right)+\left[m_{n}-m_{n-1}\right]^{+}\left(\{\omega\}_{n}^{2}\right)} .
$$

Let the set $B$ belongs to $\overline{\mathcal{F}}_{n-1}$, then

$$
\begin{aligned}
& E^{Q_{n}} \chi_{B}\left(\{\omega\}_{n-1}\right)\left[m_{n}-m_{n-1}\right]\left(\{\omega\}_{n}\right) \\
& =\int_{\bar{\Omega}_{n}^{-} \times \bar{\Omega}_{n}^{+}} \chi_{B}\left(\{\omega\}_{n-1}^{1}\right) \alpha_{n}^{0}\left(\{\omega\}_{n}^{1} ;\{\omega\}_{n}^{2}\right)\left[m_{n}-m_{n-1}\right]\left(\{\omega\}_{n}^{1}\right) \\
& \times\left[m_{n}-m_{n-1}\right]^{+}\left(\{\omega\}_{n}^{2}\right) \mathrm{d}\left[\bar{P}_{n}^{-} \times \bar{P}_{n}^{+}\right] \\
& +\int_{\bar{\Omega}_{n}^{-\times \bar{\Omega}_{n}^{+}}} \chi_{B}\left(\{\omega\}_{n-1}^{2}\right) \alpha_{n}^{0}\left(\{\omega\}_{n}^{1} ;\{\omega\}_{n}^{2}\right)\left[m_{n}-m_{n-1}\right]\left(\{\omega\}_{n}^{2}\right) \\
& \times\left[m_{n}-m_{n-1}\right]^{-}\left(\{\omega\}_{n}^{1}\right) \mathrm{d}\left[\bar{P}_{n}^{-} \times \bar{P}_{n}^{+}\right] .
\end{aligned}
$$

If to take into account the relations

$$
\begin{gathered}
{\left[m_{n}-m_{n-1}\right]\left(\{\omega\}_{n}\right)=m_{n-1}\left(\{\omega\}_{n-1}\right) a_{n}\left(\{\omega\}_{n-1}\right) \eta_{n}\left(\omega_{n}\right),} \\
{\left[m_{n}-m_{n-1}\right]^{+}\left(\{\omega\}_{n}\right)=m_{n-1}\left(\{\omega\}_{n-1}\right) a_{n}\left(\{\omega\}_{n-1}\right) \eta_{n}^{+}\left(\omega_{n}\right),} \\
{\left[m_{n}-m_{n-1}\right]^{-}\left(\{\omega\}_{n}\right)=m_{n-1}\left(\{\omega\}_{n-1}\right) a_{n}\left(\{\omega\}_{n-1}\right) \eta_{n}^{-}\left(\omega_{n}\right),}
\end{gathered}
$$

and introduce the denotations

$$
\begin{aligned}
& \theta_{1}\left(\{\omega\}_{n}^{1} ;\{\omega\}_{n}^{2}\right) \\
& =m_{n-1}\left(\{\omega\}_{n-1}^{1}\right) a_{n}\left(\{\omega\}_{n-1}^{1}\right) \times m_{n-1}\left(\{\omega\}_{n-1}^{2}\right) a_{n}\left(\{\omega\}_{n-1}^{2}\right) \alpha_{n}^{0}\left(\{\omega\}_{n}^{1} ;\{\omega\}_{n}^{2}\right), \\
& \theta_{2}\left(\{\omega\}_{n}^{1} ;\{\omega\}_{n}^{2}\right) \\
& =m_{n-1}\left(\{\omega\}_{n-1}^{1}\right) a_{n}\left(\{\omega\}_{n-1}^{1}\right) \times m_{n-1}\left(\{\omega\}_{n-1}^{2}\right) a_{n}\left(\{\omega\}_{n-1}^{2}\right) \alpha_{n}^{0}\left(\{\omega\}_{n}^{1} ;\{\omega\}_{n}^{2}\right),
\end{aligned}
$$

we obtain 


$$
\begin{aligned}
& E^{Q_{n}} \chi_{B}\left(\{\omega\}_{n-1}\right)\left[m_{n}-m_{n-1}\right]\left(\{\omega\}_{n}\right) \\
& =-\int_{\bar{\Omega}_{n}^{-} \times \Omega_{n}^{+}} \chi_{B}\left(\{\omega\}_{n-1}^{1}\right) \theta_{1}\left(\{\omega\}_{n}^{1} ;\{\omega\}_{n}^{2}\right) \eta_{n}^{-}\left(\omega_{n}^{1}\right) \eta_{n}^{+}\left(\omega_{n}^{2}\right) \mathrm{d}\left[\bar{P}_{n}^{-} \times \bar{P}_{n}^{+}\right] \\
& +\int_{\bar{\Omega}_{n}^{-} \times \bar{\Omega}_{n}^{+}} \chi_{B}\left(\{\omega\}_{n-1}^{2}\right) \theta_{2}\left(\{\omega\}_{n}^{1} ;\{\omega\}_{n}^{2}\right) \eta_{n}^{-}\left(\omega_{n}^{1}\right) \eta_{n}^{+}\left(\omega_{n}^{2}\right) \mathrm{d}\left[\bar{P}_{n}^{-} \times \bar{P}_{n}^{+}\right] \\
& =\int_{\left\{\eta_{n}\left(\omega_{n}^{1}\right) \leq 00\right\} \times\left\{\eta_{n}\left(\omega_{n}^{2}\right)>0\right\}} \mathrm{d}\left[P_{1}\left(\omega_{n}^{1}\right) \times P_{1}\left(\omega_{n}^{2}\right)\right] \eta_{n}^{-}\left(\omega_{n}^{1}\right) \eta_{n}^{+}\left(\omega_{n}^{2}\right) \\
& \times \int_{\Omega_{n-1} \times \Omega_{n-1}} \chi_{B}\left(\{\omega\}_{n-1}^{1}\right)\left[\theta_{1}\left(\{\omega\}_{n}^{1} ;\{\omega\}_{n}^{2}\right)-\theta_{1}\left(\{\omega\}_{n-1}^{2}, \omega_{n}^{1} ;\{\omega\}_{n-1}^{1}, \omega_{n}^{2}\right)\right] \\
& \times \mathrm{d}\left[P_{n-1}\left(\{\omega\}_{n-1}^{1}\right) \times P_{n-1}\left(\{\omega\}_{n-1}^{2}\right)\right] .
\end{aligned}
$$

It is evident that the expression (139) equals zero for every $B \in \overline{\mathcal{F}}_{n-1}$ if and only if as

$$
\theta_{1}\left(\{\omega\}_{n}^{1} ;\{\omega\}_{n}^{2}\right)-\theta_{1}\left(\{\omega\}_{n-1}^{2}, \omega_{n}^{1} ;\{\omega\}_{n-1}^{1}, \omega_{n}^{2}\right)=0
$$

The last equality (140) is valid if the equality

$$
\alpha_{n}^{0}\left(\{\omega\}_{n-1}^{1}, \omega_{n}^{1} ;\{\omega\}_{n-1}^{2}, \omega_{n}^{2}\right)=\alpha_{n}^{0}\left(\{\omega\}_{n-1}^{2}, \omega_{n}^{1} ;\{\omega\}_{n-1}^{1}, \omega_{n}^{2}\right)
$$

is true.

Now if for $\alpha_{n}^{2}\left(\{\omega\}_{n}^{1} ;\{\omega\}_{n}^{2}\right)>0$ satisfying the condition

$$
\int_{\bar{\Omega}_{n}^{-} \times \bar{\Omega}_{n}^{+}} \alpha_{n}^{2}\left(\{\omega\}_{n}^{1} ;\{\omega\}_{n}^{2}\right) \mathrm{d}\left[\bar{P}_{n}^{-} \times \bar{P}_{n}^{+}\right]=1
$$

to put

$$
\alpha_{n}^{0}\left(\{\omega\}_{n}^{1} ;\{\omega\}_{n}^{2}\right)=\frac{\alpha_{n}^{2}\left(\{\omega\}_{n}^{1} ;\{\omega\}_{n}^{2}\right)+\alpha_{n}^{2}\left(T_{n}\left(\{\omega\}_{n}^{1} ;\{\omega\}_{n}^{2}\right)\right)}{\varphi_{n}\left(\{\omega\}_{n}^{1} ;\{\omega\}_{n}^{2}\right)}
$$

then

$$
\begin{aligned}
Q_{n}(A)= & \int_{\bar{\Omega}_{n}^{-} \times \bar{\Omega}_{n}^{+}} \chi_{A}\left(\{\omega\}_{n}^{1}\right) \alpha_{n}^{0}\left(\{\omega\}_{n}^{1} ;\{\omega\}_{n}^{2}\right)\left[m_{n}-m_{n-1}\right]^{+}\left(\{\omega\}_{n}^{2}\right) \mathrm{d}\left[\bar{P}_{n}^{-} \times \bar{P}_{n}^{+}\right] \\
& +\int_{\bar{\Omega}_{n}^{-} \times \bar{\Omega}_{n}^{+}} \chi_{A}\left(\{\omega\}_{n}^{2}\right) \alpha_{n}^{0}\left(\{\omega\}_{n}^{1} ;\{\omega\}_{n}^{2}\right)\left[m_{n}-m_{n-1}\right]^{-}\left(\{\omega\}_{n}^{1}\right) \mathrm{d}\left[\bar{P}_{n}^{-} \times \bar{P}_{n}^{+}\right]
\end{aligned}
$$

is a probability measure on the $\sigma$-algebra $\overline{\mathcal{F}}_{n}$.

Taking into account the denotation (134) and the formula (143) we obtain that the measure

$$
\begin{aligned}
Q_{n}(A)= & \int_{\bar{\Omega}_{n}^{-} \times \bar{\Omega}_{n}^{+}} \chi_{A}\left(\{\omega\}_{n}^{1}\right) \alpha_{n}^{1}\left(\{\omega\}_{n}^{1} ;\{\omega\}_{n}^{2}\right) \frac{\left[m_{n}-m_{n-1}\right]^{+}\left(\{\omega\}_{n}^{2}\right)}{\varphi_{n}^{1}\left(\{\omega\}_{n}^{1} ;\{\omega\}_{n}^{2}\right)} \mathrm{d}\left[\bar{P}_{n}^{-} \times \bar{P}_{n}^{+}\right] \\
& +\int_{\bar{\Omega}_{n}^{\times} \times \bar{\Omega}_{n}^{+}} \chi_{A}\left(\{\omega\}_{n}^{2}\right) \alpha_{n}^{1}\left(\{\omega\}_{n}^{1} ;\{\omega\}_{n}^{2}\right) \frac{\left[m_{n}-m_{n-1}\right]^{-}\left(\{\omega\}_{n}^{1}\right)}{\varphi_{n}^{1}\left(\{\omega\}_{n}^{1} ;\{\omega\}_{n}^{2}\right)} \mathrm{d}\left[\bar{P}_{n}^{-} \times \bar{P}_{n}^{+}\right]
\end{aligned}
$$


is a probability measure on the $\sigma$-algebra $\overline{\mathcal{F}}_{n}$, where

$$
\alpha_{n}^{1}\left(\{\omega\}_{n}^{1} ;\{\omega\}_{n}^{2}\right)=\alpha_{n}^{0}\left(\{\omega\}_{n}^{1} ;\{\omega\}_{n}^{2}\right) \varphi_{n}^{1}\left(\{\omega\}_{n}^{1} ;\{\omega\}_{n}^{2}\right) .
$$

satisfy the condition

$$
\int_{\bar{\Omega}_{n}^{-} \times \bar{\Omega}_{n}^{+}} \alpha_{n}^{1}\left(\{\omega\}_{n}^{1} ;\{\omega\}_{n}^{2}\right) \mathrm{d}\left[\bar{P}_{n}^{-} \times \bar{P}_{n}^{+}\right]=1,
$$

due to the condition

$$
\int_{\bar{\Omega}_{n}^{-} \times \bar{\Omega}_{n}^{+}} \alpha_{n}^{2}\left(\{\omega\}_{n}^{1} ;\{\omega\}_{n}^{2}\right) \mathrm{d}\left[\bar{P}_{n}^{-} \times \bar{P}_{n}^{+}\right]=1 .
$$

So, we described the contraction of the martingale measure $Q$ on the $\sigma$-algebra $\mathcal{F}_{n}$ for which $E^{Q_{n}}\left\{m_{n} \mid \mathcal{F}_{n-1}\right\}=m_{n-1}$. It has the representation (145) with the strictly positive random values $\alpha_{n}^{1}\left(\{\omega\}_{n}^{1} ;\{\omega\}_{n}^{2}\right), \alpha_{n}^{2}\left(\{\omega\}_{n}^{1} ;\{\omega\}_{n}^{2}\right)$ satisfying conditions (147), (148).

Since $\Omega_{1}^{0}$ is a separable metric space, then it has an exhaustive decomposition. This is true for $\Omega_{n}$ which is also separable metric space for every $n=\overline{2, N}$. On the probability space $\left\{\bar{\Omega}_{n}^{-} \times \bar{\Omega}_{n}^{+}, \overline{\mathcal{F}}_{n}^{-} \times \overline{\mathcal{F}}_{n}^{+}, \bar{P}_{n}^{-} \times \bar{P}_{n}^{+}\right\}$, for every integrable finite valued random value $f\left(\{\omega\}_{n}^{1} ;\{\omega\}_{n}^{2}\right)$ the sequence $E^{\bar{\mu}_{n}}\left\{f\left(\{\omega\}_{n}^{1} ;\{\omega\}_{n}^{2}\right) \mid \overline{\mathcal{F}}_{m}\right\}$ converges to $f\left(\{\omega\}_{n}^{1} ;\{\omega\}_{n}^{2}\right)$ with probability one, as $m \rightarrow \infty$, since it is a regular martingale. It is evident that for those $B_{m, k s}$ for which $\bar{\mu}_{n}\left(B_{m, k s}\right) \neq 0, \bar{\mu}_{n}=\bar{P}_{n}^{-} \times \bar{P}_{n}^{+}$,

$$
\begin{aligned}
& E^{\bar{\mu}_{n}}\left\{f\left(\{\omega\}_{n}^{1},\{\omega\}_{n}^{2}\right) \mid \overline{\mathcal{F}}_{m}\right\} \\
& =\frac{\int_{B_{m, k s}} f\left(\{\omega\}_{n}^{1} ;\{\omega\}_{n}^{2}\right) \mathrm{d} \bar{\mu}_{n}}{\bar{\mu}_{n}\left(B_{m, k s}\right)},\left(\{\omega\}_{n}^{1} ;\{\omega\}_{n}^{2}\right) \in B_{m, k s} .
\end{aligned}
$$

Denote $D_{0}=\bigcup_{m, k, s, \mu\left(B_{m, k s}\right)=0} B_{m, k s}$. It is evident that $\bar{\mu}_{n}\left(D_{0}\right)=0$. For every $\left(\{\omega\}_{n}^{1} ;\{\omega\}_{n}^{2}\right) \in \bar{\Omega}_{n}^{-} \times \bar{\Omega}_{n}^{+} \backslash D_{0}$, the formula (149) is well defined and is finite. Let $D_{1}$ be the subset of the set $\bar{\Omega}_{n}^{-} \times \bar{\Omega}_{n}^{+} \backslash D_{0}$, where the limit of the left hand side of the formula (149) does not exists. Then, $\bar{\mu}_{n}\left(D_{1}\right)=0$. For every $\left(\{\omega\}_{n}^{1} ;\{\omega\}_{n}^{2}\right) \in \bar{\Omega}_{n}^{-} \times \bar{\Omega}_{n}^{+} \backslash\left(D_{0} \cup D_{1}\right)$, the right hand side of the formula (149) converges to $f\left(\{\omega\}_{n}^{1} ;\{\omega\}_{n}^{2}\right)$. For $\left(\{\omega\}_{n}^{1} ;\{\omega\}_{n}^{2}\right) \in \bar{\Omega}_{n}^{-} \times \bar{\Omega}_{n}^{+} \backslash\left(D_{0} \cup D_{1}\right)$, denote $A_{m}=A_{m}\left(\{\omega\}_{n}^{1} ;\{\omega\}_{n}^{2}\right)^{n}$ those set $B_{m, k s}$ for which $\left(\{\omega\}_{n}^{1} ;\{\omega\}_{n}^{2}\right) \in B_{m, k s}$ for a certain $k, s$. Then, for every integrable finite valued $f\left(\{\omega\}_{n}^{1} ;\{\omega\}_{n}^{2}\right)$

$$
\lim _{m \rightarrow \infty} \frac{\int_{A_{m}} f\left(\{\omega\}_{n}^{1} ;\{\omega\}_{n}^{2}\right) \mathrm{d} \bar{\mu}_{n}}{\bar{\mu}_{n}\left(A_{m}\right)}=f\left(\{\omega\}_{n}^{1} ;\{\omega\}_{n}^{2}\right) .
$$

Choose the sequence 


$$
\begin{aligned}
& \alpha_{n}^{2, \varepsilon_{m}}\left(\{\omega\}_{n}^{1} ;\{\omega\}_{n}^{2}\right) \\
& =\left(1-\varepsilon_{m}\right) \frac{\chi_{A_{m}}\left(\{\omega\}_{n}^{1} ;\{\omega\}_{n}^{2}\right)}{\bar{\mu}_{n}\left(A_{m}\right)}+\varepsilon_{m} \frac{\chi_{\bar{\Omega}_{n}^{-} \times \bar{\Omega}_{n}^{+} \backslash A_{m}}\left(\{\omega\}_{n}^{1} ;\{\omega\}_{n}^{2}\right)}{\bar{\mu}_{n}\left(\bar{\Omega}_{n}^{-} \times \bar{\Omega}_{n}^{+} \backslash A_{m}\right)},
\end{aligned}
$$

where $0<\varepsilon_{m}<1, \lim _{m \rightarrow \infty} \varepsilon_{m}=0$. Then the sequence $\alpha_{n}^{2, \varepsilon_{m}}\left(\{\omega\}_{n}^{1} ;\{\omega\}_{n}^{2}\right)$ satisfy the condition (148). Let us consider the sequence

$$
\begin{aligned}
& \alpha_{n}^{0, \varepsilon_{m}}\left(\{\omega\}_{n}^{1} ;\{\omega\}_{n}^{2}\right) \\
& =\frac{1}{\varphi_{n}\left(\{\omega\}_{n}^{1} ;\{\omega\}_{n}^{2}\right)}\left[\left(1-\varepsilon_{m}\right) \frac{\chi_{A_{m}}\left(\{\omega\}_{n}^{1} ;\{\omega\}_{n}^{2}\right)}{\bar{\mu}_{n}\left(A_{m}\right)}\right. \\
& \left.+\varepsilon_{m} \frac{\chi_{\bar{\Omega}_{n}^{-} \times \bar{\Omega}_{n}^{+} \backslash A_{m}}\left(\{\omega\}_{n}^{1} ;\{\omega\}_{n}^{2}\right)}{\bar{\mu}_{n}\left(\bar{\Omega}_{n}^{-} \times \bar{\Omega}_{n}^{+} \backslash A_{m}\right)}\right] \\
& +\frac{1}{\varphi_{n}\left(\{\omega\}_{n}^{1} ;\{\omega\}_{n}^{2}\right)}\left[\left(1-\varepsilon_{m}\right) \frac{\chi_{A_{m}}\left(T_{n}\left(\{\omega\}_{n}^{1} ;\{\omega\}_{n}^{2}\right)\right)}{\bar{\mu}_{n}\left(A_{m}\right)}\right. \\
& \left.+\varepsilon_{m} \frac{\chi_{\bar{\Omega}_{n}^{-} \times \bar{\Omega}_{n}^{+} \backslash A_{m}}\left(T_{n}\left(\{\omega\}_{n}^{1} ;\{\omega\}_{n}^{2}\right)\right)}{\bar{\mu}_{n}\left(\bar{\Omega}_{n}^{-} \times \bar{\Omega}_{n}^{+} \backslash A_{m}\right)}\right] .
\end{aligned}
$$

Then the contraction of the sequence of martingale measures $Q_{n}^{\varepsilon_{m}}$ generated by sequence (152) on the $\sigma$-algebra $\mathcal{F}_{n}$ is given by the formula

$$
\begin{aligned}
& E^{Q_{n}^{\varepsilon_{m}}} f(\omega) \\
& =\int_{\bar{\Omega}_{n}^{-} \bar{\Omega}_{n}^{+}} f\left(\{\omega\}_{n}^{1}\right) \alpha_{n}^{0, \varepsilon_{m}}\left(\{\omega\}_{n}^{1} ;\{\omega\}_{n}^{2}\right)\left[m_{n}-m_{n-1}\right]^{+}\left(\{\omega\}_{n}^{2}\right) \mathrm{d} \bar{\mu}_{n} \\
& +\int_{\bar{\Omega}_{n}^{-} \bar{\Omega}_{n}^{+}} f\left(\{\omega\}_{n}^{2}\right) \alpha_{n}^{0, \varepsilon_{m}}\left(\{\omega\}_{n}^{1} ;\{\omega\}_{n}^{2}\right)\left[m_{n}-m_{n-1}\right]^{-}\left(\{\omega\}_{n}^{1}\right) \mathrm{d} \bar{\mu}_{n} \\
& =\left(1-\varepsilon_{m}\right) \frac{\int_{A_{m}} f\left(\{\omega\}_{n}^{1}\right) v_{n}^{1}\left(\{\omega\}_{n}^{1} ;\{\omega\}_{n}^{2}\right) \mathrm{d} \bar{\mu}_{n}}{\bar{\mu}_{n}\left(A_{m}\right)} \\
& +\left(1-\varepsilon_{m}\right) \frac{\int_{A_{m}} f\left(\{\omega\}_{n}^{2}\right) v_{n}^{2}\left(\{\omega\}_{n}^{1} ;\{\omega\}_{n}^{2}\right) \mathrm{d} \bar{\mu}_{n}}{\bar{\mu}_{n}\left(A_{m}\right)} \\
& +\varepsilon_{m} \frac{\int_{n}^{-} \times \bar{\Omega}_{n}^{+} \backslash A_{m}}{f\left(\{\omega\}_{n}^{1}\right) v_{n}^{1}\left(\{\omega\}_{n}^{1} ;\{\omega\}_{n}^{2}\right) \mathrm{d} \bar{\mu}_{n}} \\
& \bar{\mu}_{n}\left(\bar{\Omega}_{n}^{-} \times \bar{\Omega}_{n}^{+} \backslash A_{m}\right) \\
& +\varepsilon_{m} \frac{\bar{\Omega}_{n}^{-} \times \bar{\Omega}_{n}^{+} \backslash A_{m}}{f\left(\{\omega\}_{n}^{2}\right) v_{n}^{2}\left(\{\omega\}_{n}^{1} ;\{\omega\}_{n}^{2}\right) \mathrm{d} \bar{\mu}_{n}} \\
& \bar{\mu}_{n}\left(\bar{\Omega}_{n}^{-} \times \bar{\Omega}_{n}^{+} \backslash A_{m}\right)
\end{aligned}
$$




$$
\begin{aligned}
& +\left(1-\varepsilon_{m}\right) \frac{\int_{\bar{\Omega}_{n}^{-} \times \bar{\Omega}_{n}^{+}} \chi_{A_{m}}\left(T_{n}\left(\{\omega\}_{n}^{1} ;\{\omega\}_{n}^{2}\right)\right) f\left(\{\omega\}_{n}^{1}\right) v_{n}^{1}\left(\{\omega\}_{n}^{1} ;\{\omega\}_{n}^{2}\right) \mathrm{d} \bar{\mu}_{n}}{\bar{\mu}_{n}\left(A_{m}\right)} \\
& +\left(1-\varepsilon_{m}\right) \frac{\int_{\bar{\Omega}_{n}^{-} \times \bar{\Omega}_{n}^{+}} \chi_{A_{m}}\left(T_{n}\left(\{\omega\}_{n}^{1} ;\{\omega\}_{n}^{2}\right)\right) f\left(\{\omega\}_{n}^{2}\right) v_{n}^{2}\left(\{\omega\}_{n}^{1} ;\{\omega\}_{n}^{2}\right) \mathrm{d} \bar{\mu}_{n}}{\bar{\mu}_{n}\left(A_{m}\right)} \\
& +\varepsilon_{m} \frac{\int_{\bar{\Omega}_{n}^{-} \times \bar{\Omega}_{n}^{+}} \chi_{\bar{\Omega}_{n}^{-} \times \bar{\Omega}_{n}^{+} \backslash A_{m}}\left(T_{n}\left(\{\omega\}_{n}^{1} ;\{\omega\}_{n}^{2}\right)\right) f\left(\{\omega\}_{n}^{1}\right) v_{n}^{1}\left(\{\omega\}_{n}^{1} ;\{\omega\}_{n}^{2}\right) \mathrm{d} \bar{\mu}_{n}}{\bar{\mu}_{n}\left(\bar{\Omega}_{n}^{-} \times \bar{\Omega}_{n}^{+} \backslash A_{m}\right)} \\
& +\varepsilon_{m} \frac{\int_{-} \times \bar{\Omega}_{n}^{+}}{\chi_{\bar{\Omega}_{n}^{-} \times \bar{\Omega}_{n}^{+} \backslash A_{m}}\left(T_{n}\left(\{\omega\}_{n}^{1} ;\{\omega\}_{n}^{2}\right)\right) f\left(\{\omega\}_{n}^{2}\right) v_{n}^{2}\left(\{\omega\}_{n}^{1} ;\{\omega\}_{n}^{2}\right) \mathrm{d} \bar{\mu}_{n}} \\
& \bar{\mu}_{n}\left(\bar{\Omega}_{n}^{-} \times \bar{\Omega}_{n}^{+} \backslash A_{m}\right)
\end{aligned}
$$

Due to the invariance of the measure $\bar{\mu}_{n}$ relative to the transformation $T_{n}$ we have

$$
\begin{aligned}
& \left(1-\varepsilon_{m}\right) \frac{\int_{\bar{\Omega}_{n}^{-} \times \bar{\Omega}_{n}^{+}} \chi_{A_{m}}\left(T_{n}\left(\{\omega\}_{n}^{1} ;\{\omega\}_{n}^{2}\right)\right) f\left(\{\omega\}_{n}^{1}\right) v_{n}^{1}\left(\{\omega\}_{n}^{1} ;\{\omega\}_{n}^{2}\right) \mathrm{d} \bar{\mu}_{n}}{\bar{\mu}_{n}\left(A_{m}\right)} \\
& +\left(1-\varepsilon_{m}\right) \frac{\int_{\bar{\Omega}_{n}^{\times} \times \bar{\Omega}_{n}^{+}} \chi_{A_{m}}\left(T_{n}\left(\{\omega\}_{n}^{1} ;\{\omega\}_{n}^{2}\right)\right) f\left(\{\omega\}_{n}^{2}\right) v_{n}^{2}\left(\{\omega\}_{n}^{1} ;\{\omega\}_{n}^{2}\right) \mathrm{d} \bar{\mu}_{n}}{\bar{\mu}_{n}\left(A_{m}\right)} \\
& =\left(1-\varepsilon_{m}\right) \frac{\int_{A_{m}} f\left(T_{n}^{1}\left(\{\omega\}_{n}^{1}\right)\right) v_{n}^{1}\left(T_{n}\left(\{\omega\}_{n}^{1} ;\{\omega\}_{n}^{2}\right)\right) \mathrm{d} \bar{\mu}_{n}}{\bar{\mu}_{n}\left(A_{m}\right)} \\
& +\left(1-\varepsilon_{m}\right) \frac{\int_{A_{m}} f\left(T_{n}^{2}\left(\{\omega\}_{n}^{2}\right)\right) v_{n}^{2}\left(T_{n}\left(\{\omega\}_{n}^{1} ;\{\omega\}_{n}^{2}\right)\right) \mathrm{d} \bar{\mu}_{n}}{\bar{\mu}\left(A_{m}\right)} .
\end{aligned}
$$

From the equalities (153), (154) it follows that

$$
\begin{aligned}
& \lim _{m \rightarrow \infty} E^{Q_{n}^{\varepsilon_{m}}} f_{n}\left(\{\omega\}_{n}\right) \\
& =f\left(\{\omega\}_{n}^{1}\right) v_{n}^{1}\left(\{\omega\}_{n}^{1} ;\{\omega\}_{n}^{2}\right)+f\left(\{\omega\}_{n}^{2}\right) v_{n}^{2}\left(\{\omega\}_{n}^{1} ;\{\omega\}_{n}^{2}\right) \\
& +f\left(T_{n}^{1}\left(\{\omega\}_{n}^{1}\right)\right) v_{n}^{1}\left(T_{n}\left(\{\omega\}_{n}^{1} ;\{\omega\}_{n}^{2}\right)\right) \\
& +f\left(T_{n}^{2}\left(\{\omega\}_{n}^{2}\right)\right) v_{n}^{2}\left(T_{n}\left(\{\omega\}_{n}^{1} ;\{\omega\}_{n}^{2}\right)\right), n=\overline{1, N} .
\end{aligned}
$$

Theorem 9 is proved.

Theorem 10. On the probability space $\{\Omega, \mathcal{F}, P\}$ with the filtration $\mathcal{F}_{n}$ on it, let $\Omega_{1}^{0}$ be a complete separable metric space. Suppose that $f_{n}\left(\{\omega\}_{n}\right)$ is a nonnegative integrable $\mathcal{F}_{n}$-measurable random value, satisfying the condition $E^{Q^{n}} f_{n}\left(\{\omega\}_{n}\right) \leq 1, Q^{n} \in M_{n}$. Then, there exists a $F_{n-1}$-measurable random value $\alpha_{n}$, depending on $f_{n}\left(\{\omega\}_{n}\right)$, such that

$$
f_{n}\left(\{\omega\}_{n}\right) \leq 1+\alpha_{n}\left(\{\omega\}_{n-1}\right)\left[m_{n}-m_{n-1}\right]\left(\{\omega\}_{n}\right),\{\omega\}_{n} \in \Omega_{n} .
$$


Proof. First, let us consider the case $n=1$. From Theorem 9, we have the inequality

$$
\begin{aligned}
& f_{1}\left(\omega_{1}\right) \frac{\left[m_{1}-1\right]^{+}\left(\omega_{2}\right)}{\left[m_{1}-1\right]^{-}\left(\omega_{1}\right)+\left[m_{1}-1\right]^{+}\left(\omega_{2}\right)} \\
& +f_{1}\left(\omega_{2}\right) \frac{\left[m_{1}-1\right]^{-}\left(\omega_{1}\right)}{\left[m_{1}-1\right]^{-}\left(\omega_{1}\right)+\left[m_{1}-1\right]^{+}\left(\omega_{2}\right)} \leq 1,\left(\omega_{1}, \omega_{2}\right) \in \Omega_{1}^{-} \times \Omega_{1}^{+},
\end{aligned}
$$

where $\Omega_{1}^{-}=\left\{\omega_{1} \in \Omega_{1}^{0},\left[m_{1}-1\right]\left(\omega_{1}\right) \leq 0\right\}, \Omega_{1}^{+}=\left\{\omega_{2} \in \Omega_{1}^{0},\left[m_{1}-1\right]\left(\omega_{2}\right)>0\right\}$.

Let us put $\eta_{1}(\omega)=\left[m_{1}-1\right](\omega)$. Then, the formula (157) is written in the form $f_{1}\left(\omega_{1}\right) \frac{\eta_{1}^{+}\left(\omega_{2}\right)}{\eta_{1}^{-}\left(\omega_{1}\right)+\eta_{1}^{+}\left(\omega_{2}\right)}+\frac{\eta_{1}^{-}\left(\omega_{1}\right)}{\eta_{1}^{-}\left(\omega_{1}\right)+\eta_{1}^{+}\left(\omega_{2}\right)} f_{1}\left(\omega_{2}\right) \leq 1, \omega_{1} \in \Omega_{1}^{-}, \omega_{2} \in \Omega_{1}^{+}$.

From the inequalities (158), we obtain the inequalities

$$
\begin{gathered}
f_{1}\left(\omega_{2}\right) \leq 1+\frac{1-f_{1}\left(\omega_{1}\right)}{\eta_{1}^{-}\left(\omega_{1}\right)} \eta_{1}^{+}\left(\omega_{2}\right), \\
\eta_{1}^{-}\left(\omega_{1}\right)>0, \eta_{1}^{+}\left(\omega_{2}\right)>0, \omega_{1} \in \Omega_{1}^{-}, \omega_{2} \in \Omega_{1}^{+} .
\end{gathered}
$$

Two cases are possible: a) for all $\omega_{1} \in \Omega_{n}^{-}, f_{1}\left(\omega_{1}\right) \leq 1$; b) there exists $\omega_{1} \in \Omega_{1}^{-}$such that $f_{1}\left(\omega_{1}\right)>1$. First, let us consider the case a).

Since the inequalities (159) are valid for every value $\frac{1-f_{1}\left(\omega_{1}\right)}{\eta_{1}^{-}\left(\omega_{1}\right)}$, as $\eta_{1}^{-}\left(\omega_{1}\right)>0$, and $f_{1}\left(\omega_{1}\right) \leq 1, \omega_{1} \in \Omega_{1}^{-}$, then, if to denote

$$
\alpha_{1}=\inf _{\left\{\omega_{1}, \eta_{1}^{-}\left(\omega_{1}\right)>0\right\}} \frac{1-f_{1}\left(\omega_{1}\right)}{\eta_{1}^{-}\left(\omega_{1}\right)}
$$

we have $0 \leq \alpha_{1}<\infty$ and

$$
f_{1}\left(\omega_{2}\right) \leq 1+\alpha_{1} \eta_{1}^{+}\left(\omega_{2}\right), \eta_{1}^{+}\left(\omega_{2}\right)>0, \omega_{2} \in \Omega_{1}^{+} .
$$

From the definition of $\alpha_{1}$, we obtain the inequalities

$$
f_{1}\left(\omega_{1}\right) \leq 1-\alpha_{1} \eta_{1}^{-}\left(\omega_{1}\right), \eta_{1}^{-}\left(\omega_{1}\right)>0, \omega_{1} \in \Omega_{1}^{-} .
$$

Now, if $\eta_{1}^{-}\left(\omega_{1}\right)=0$ for some $\omega_{1} \in \Omega_{1}^{-}$, then in this case $f_{1}\left(\omega_{1}\right) \leq 1$. All these inequalities give the inequalities

$$
f_{1}(\omega) \leq 1+\alpha_{1} \eta_{1}(\omega), \omega \in \Omega_{1}^{-} \cup \Omega_{1}^{+} .
$$

Consider the case b). From the inequality (159), we obtain the inequalities

$$
\begin{gathered}
f_{1}\left(\omega_{2}\right) \leq 1-\frac{1-f_{1}\left(\omega_{1}\right)}{-\eta_{1}^{-}\left(\omega_{1}\right)} \eta_{1}^{+}\left(\omega_{2}\right), \\
\eta_{1}^{-}\left(\omega_{1}\right)>0, \eta_{1}^{+}\left(\omega_{2}\right)>0, \omega_{1} \in \Omega_{1}^{-}, \omega_{2} \in \Omega_{1}^{+} .
\end{gathered}
$$

The inequalities (165) give the inequalities

$$
\frac{1-f_{1}\left(\omega_{1}\right)}{-\eta_{1}^{-}\left(\omega_{1}\right)} \leq \inf _{\left\{\omega_{2}, \eta_{1}^{+}\left(\omega_{2}\right)>0\right\}} \frac{1}{\eta_{1}^{+}\left(\omega_{2}\right)}<\infty, \eta_{1}^{-}\left(\omega_{1}\right)>0, \omega_{1} \in \Omega_{1}^{-} .
$$


Let us define $\alpha_{1}=\sup _{\left\{\omega_{1}, \eta_{1}^{-}\left(\omega_{1}\right)>0\right\}} \frac{1-f_{1}\left(\omega_{1}\right)}{-\eta_{1}^{-}\left(\omega_{1}\right)}<\infty$. Then, from (165) we obtain the inequalities

$$
f_{1}\left(\omega_{2}\right) \leq 1-\alpha_{1} \eta_{1}^{+}\left(\omega_{2}\right), \eta_{1}^{+}\left(\omega_{2}\right)>0, \omega_{2} \in \Omega_{1}^{+} .
$$

From the definition of $\alpha_{1}$, we have the inequalities

$$
f_{1}\left(\omega_{1}\right) \leq 1+\alpha_{1} \eta_{1}^{-}\left(\omega_{1}\right), \eta_{1}^{-}\left(\omega_{1}\right)>0, \omega_{1} \in \Omega_{1}^{-} .
$$

The inequalities (168), (169) give the inequalities

$$
f_{1}(\omega) \leq 1-\alpha_{1} \eta_{1}(\omega), \omega \in \Omega_{1}^{-} \cup \Omega_{1}^{+} .
$$

Theorem 10 in the case $n=1$ is proved, since the set $\Omega_{1}^{-} \cup \Omega_{1}^{+}$has the probability one.

Now let us consider the case of arbitrary $2 \leq n \leq N$. In this case we have the inequality

$$
\begin{aligned}
& f\left(\{\omega\}_{n}^{1}\right) v_{n}^{1}\left(\{\omega\}_{n}^{1} ;\{\omega\}_{n}^{2}\right)+f\left(\{\omega\}_{n}^{2}\right) v_{n}^{2}\left(\{\omega\}_{n}^{1} ;\{\omega\}_{n}^{2}\right) \\
& +f\left(T_{n}^{1}\left(\{\omega\}_{n}^{1}\right)\right) v_{n}^{1}\left(T_{n}\left(\{\omega\}_{n}^{1} ;\{\omega\}_{n}^{2}\right)\right)+f\left(T_{n}^{2}\left(\{\omega\}_{n}^{2}\right)\right) v_{n}^{2}\left(T_{n}\left(\{\omega\}_{n}^{1} ;\{\omega\}_{n}^{2}\right)\right) \leq 1 .
\end{aligned}
$$

Let us put in this inequality $\{\omega\}_{n-1}^{1}=\{\omega\}_{n-1}^{2}=\{\omega\}_{n-1}$, then the inequality (171) is transformed into the inequality

$$
\begin{aligned}
& f_{n}\left(\{\omega\}_{n-1}, \omega_{n}^{1}\right) \frac{\eta_{n}^{+}\left(\omega_{n}^{2}\right)}{\eta_{n}^{-}\left(\omega_{n}^{1}\right)+\eta_{n}^{+}\left(\omega_{n}^{2}\right)}+\frac{\eta_{n}^{-}\left(\omega_{n}^{1}\right)}{\eta_{n}^{-}\left(\omega_{n}^{1}\right)+\eta_{n}^{+}\left(\omega_{n}^{2}\right)} f_{n}\left(\{\omega\}_{n-1}, \omega_{n}^{2}\right) \leq 1, \\
& \left(\{\omega\}_{n-1}, \omega_{n}^{1}\right) \in \Omega_{n}^{-},\left(\{\omega\}_{n-1}, \omega_{n}^{2}\right) \in \Omega_{n}^{+} .
\end{aligned}
$$

Taking into account the first part of the proof of Theorem 10 from the inequality (172) we obtain

$$
f\left(\{\omega\}_{n}\right) \leq 1+\alpha_{1} \eta_{n}\left(\omega_{n}\right)=1+\frac{\alpha_{1}\left[m_{n}-m_{n-1}\right]\left(\{\omega\}_{n}\right)}{m_{n-1}\left(\{\omega\}_{n-1}\right) a_{n}\left(\{\omega\}_{n-1}\right)},
$$

where the constant $\alpha_{1}$ is the same as in the first part of the proof of Theorem 10. Theorem 10 is completely proved.

Theorem 11. On the probability space $\{\Omega, \mathcal{F}, P\}$ with the filtration $\mathcal{F}_{n}$ on it, let $\Omega_{1}^{0}$ be a complete separable metric space. Then, every nonnegative super-martingale $\left\{f_{n}, \mathcal{F}_{n}\right\}_{n=0}^{\infty}$ is a local regular one.

Proof. Without loss of generality, we assume that $f_{n} \geq d_{0}>0$. From the last fact, we obtain

$$
E^{Q^{n}} \frac{f_{n}}{f_{n-1}} \leq 1, Q^{n} \in M_{n}, n=\overline{1, \infty} .
$$

The inequalities (174) and Theorems 4, 10 prove Theorem 11.

Theorem 12. On the probability space $\{\Omega, \mathcal{F}, P\}$ with the filtration $\mathcal{F}_{n}$ on it, let $\Omega_{1}^{0}$ be a complete separable metric space. Then, every bounded from below super-martingale $\left\{f_{n}, \mathcal{F}_{n}\right\}_{n=0}^{\infty}$ is a local regular one.

Proof. Since the super-martingale $\left\{f_{n}, \mathcal{F}_{n}\right\}_{n=0}^{\infty}$ is bounded from below, then 
there exists a real number $C_{0}$ such that $f_{n}+C_{0}>0$. If to consider the super-martingale $\left\{f_{n}+C_{0}, \mathcal{F}_{n}\right\}_{n=0}^{\infty}$, then all conditions of Theorem 11 are true. Theorem 12 is proved.

Theorem 13. On the probability space $\{\Omega, \mathcal{F}, P\}$ with the filtration $\mathcal{F}_{n}$ on it, let $\Omega_{1}^{0}$ be a complete separable metric space. Suppose that evolution of the risk asset is defined by the formula (114) and non risk asset evolve by the law $B_{n}=1, n=\overline{0, N}$. If the nonnegative payment function $f_{N}$ is $\mathcal{F}_{N}$ measurable integrable random value relative to every martingale measure and satisfying conditions Theorem 16 from [5], then the fair price of super-hedge is given by the formula

$$
\sup _{Q \in M} \int_{\Omega} f_{N} \mathrm{~d} Q=\sup _{v \in \mathcal{V}} \int_{\Omega} f_{N} \mathrm{~d} v_{v} .
$$

Proof. The left part of the formula (175) for the super-hedge is true (see: [5]), since for the super-martingale $\left\{\underset{P \in M}{\operatorname{esssup}} E^{P}\left\{\xi \mid \mathcal{F}_{n}\right\}, F_{n}\right\}_{n=0}^{N}$ the optional decomposition is true due to Theorem 11. The left hand side of the formula (175) equals right hand side one due to Theorem 8 . Theorem 13 is proved.

\section{Description of Local Regular Super-Martingales Relative to a Regular Set of Measures}

In this section, we give the description of local regular super-martingales.

Theorem 14. On the measurable space $\{\Omega, \mathcal{F}\}$ with the filtration $\mathcal{F}_{n}$ on it, let $M$ be a regular set of measures. If $\left\{f_{m}, \mathcal{F}_{m}\right\}_{m=0}^{\infty}$ is an adapted random process, satisfying the conditions

$$
f_{m} \leq f_{m-1}, E^{P} \xi\left|f_{m}\right|<\infty, P \in M, m=\overline{1, \infty}, \quad \xi \in A_{0},
$$

then the random process

$$
\left\{f_{m} E^{P}\left\{\xi \mid \mathcal{F}_{m}\right\}, \mathcal{F}_{m}\right\}_{m=0}^{\infty}, P \in M,
$$

is a local regular super-martingale relative to the regular set of measures $M$.

Proof. Due to Theorem 3, the random process $\left\{E^{P}\left\{\xi \mid \mathcal{F}_{m}\right\}, \mathcal{F}_{m}\right\}_{m=0}^{\infty}$ is a martingale relative to the regular set of measures $M$. Therefore,

$$
\begin{aligned}
& f_{m-1} E^{P}\left\{\xi \mid \mathcal{F}_{m-1}\right\}-E^{P}\left\{f_{m} E^{P}\left\{\xi \mid \mathcal{F}_{m}\right\} \mid \mathcal{F}_{m-1}\right\} \\
& =E^{P}\left\{\left(f_{m-1}-f_{m}\right) E^{P}\left\{\xi \mid \mathcal{F}_{m}\right\} \mid \mathcal{F}_{m-1}\right\}, m=\overline{1, \infty} .
\end{aligned}
$$

So, if to put $\bar{g}_{m}^{0}=\left(f_{m-1}-f_{m}\right) E^{P}\left\{\xi \mid \mathcal{F}_{m}\right\}, m=\overline{1, \infty}$, then $\bar{g}_{m}^{0} \geq 0$, it is $\mathcal{F}_{m}$ measurable and $E^{P} \bar{g}_{m}^{0} \leq E^{P} \xi\left(\left|f_{m-1}\right|+\left|f_{m}\right|\right)<\infty$. Due to Theorem 1, we obtain the proof of Theorem 14 .

Corollary 1. If $f_{m}=\alpha, m=\overline{1, \infty}, \alpha \in R^{1}, \xi \in A_{0}$, then $\left\{\alpha E^{P}\left\{\xi \mid \mathcal{F}_{m}\right\}, \mathcal{F}_{m}\right\}_{m=0}^{\infty}$ is a local regular martingale. Assume that $\xi=1$, then $\left\{f_{m}, \mathcal{F}_{m}\right\}_{m=0}^{\infty}$ is a local regular super-martingale relative to the regular set of measures $M$.

Denote $F_{0}$ the set of adapted processes

$$
F_{0}=\left\{f=\left\{f_{m}\right\}_{m=0}^{\infty}, P\left(\left|f_{m}\right|<\infty\right)=1, P \in M, f_{m} \leq f_{m-1}\right\} .
$$


For every $\xi \in A_{0}$, let us introduce the set of adapted processes

$$
L_{\xi}=\left\{\bar{f}=\left\{f_{m} E^{P}\left\{\xi \mid \mathcal{F}_{m}\right\}\right\}_{m=0}^{\infty},\left\{f_{m}\right\}_{m=0}^{\infty} \in F_{0}, E^{P} \xi\left|f_{m}\right|<\infty, P \in M\right\},
$$

and

$$
V=\bigcup_{\xi \in A_{0}} L_{\xi} .
$$

Corollary 2. Every random process from the set $K$, where

$$
K=\left\{\sum_{i=1}^{m} C_{i} \bar{f}_{i}, \bar{f}_{i} \in V, C_{i} \geq 0, i=\overline{1, m}, m=\overline{1, \infty}\right\},
$$

is a local regular super-martingale relative to the regular set of measures $M$ on the measurable space $\{\Omega, \mathcal{F}\}$ with the filtration $\mathcal{F}_{n}$ on it.

Proof. The proof is evident.

Theorem 15. On the measurable space $\{\Omega, \mathcal{F}\}$ with the filtration $\mathcal{F}_{n}$ on it, let $M$ be a regular set of measures. Suppose that $\left\{f_{m}, \mathcal{F}_{m}\right\}_{m=0}^{\infty}$ is a nonnegative uniformly integrable super-martingale relative to the set of measures $M$, then the necessary and sufficient conditions for it to be a local regular one is belonging it to the set $K$.

Proof. The necessity. It is evident that if $\left\{f_{m}, \mathcal{F}_{m}\right\}_{m=0}^{\infty}$ belongs to $K$, then it is a local regular super-martingale.

The sufficiency. Suppose that $\left\{f_{m}, \mathcal{F}_{m}\right\}_{m=0}^{\infty}$ is a nonnegative uniformly integrable local regular super-martingale. Then, there exists a nonnegative adapted process $\left\{\bar{g}_{m}^{0}\right\}_{m=1}^{\infty}, E^{P} \bar{g}_{m}^{0}<\infty, m=\overline{1, \infty}$, and a martingale $\left\{M_{m}, \mathcal{F}_{m}\right\}_{m=0}^{\infty}$, such that

$$
f_{m}=M_{m}-\sum_{i=1}^{m} \bar{g}_{i}^{0}, m=\overline{0, \infty} .
$$

Then, $M_{m} \geq 0, m=\overline{0, \infty}, E^{P} M_{m}<\infty, P \in M$. Since $0<E^{P} M_{m}=f_{0}<\infty$, we have $E^{P} \sum_{i=1}^{m} \bar{g}_{i}^{0}<f_{0}$. Let us put $g_{\infty}=\lim _{m \rightarrow \infty} \sum_{i=1}^{m} \bar{g}_{i}^{0}$. Using the uniform integrability of $\left\{f_{m}, \mathcal{F}_{m}\right\}_{m=0}^{\infty}$, we can pass to the limit in the equality

$$
E^{P}\left(f_{m}+\sum_{i=1}^{m} \bar{g}_{i}^{0}\right)=f_{0}, P \in M,
$$

as $m \rightarrow \infty$. Passing to the limit in the last equality, as $m \rightarrow \infty$, we obtain

$$
E^{P}\left(f_{\infty}+g_{\infty}\right)=f_{0}, P \in M .
$$

Introduce into consideration a random value $\xi=\frac{f_{\infty}+g_{\infty}}{f_{0}}$. Then,

$E^{P} \xi=1, P \in M$. From here, we obtain that $\xi \in A_{0}$ and

$$
M_{m}=f_{0} E^{P}\left\{\xi \mid \mathcal{F}_{m}\right\}, m=\overline{0, \infty} .
$$

Let us put $\bar{f}_{m}^{2}=-\sum_{i=1}^{m} \bar{g}_{i}^{0}$. It is easy to see that the adapted random process $\bar{f}_{2}=\left\{\bar{f}_{m}^{2}, \mathcal{F}_{m}\right\}_{m=0}^{\infty}$ belongs to $\mathcal{F}_{0}$. Therefore, for the super-martingale 
$f=\left\{f_{m}, \mathcal{F}_{m}\right\}_{m=0}^{\infty}$ the representation

$$
f=\bar{f}_{1}+\bar{f}_{2},
$$

is valid, where $\bar{f}_{1}=\left\{f_{0} E^{P}\left\{\xi \mid \mathcal{F}_{m}\right\}, \mathcal{F}_{m}\right\}_{m=0}^{\infty}$ belongs to $L_{\xi}$ with $\xi=\frac{f_{\infty}+g_{\infty}}{f_{0}}$ and $f_{m}^{1}=f_{0}, m=\overline{0, \infty}$. The same is valid for $\bar{f}_{2}$ with $\xi=1$. This implies that $f$ belongs to the set $K$. Theorem 15 is proved.

Theorem 16. On the measurable space $\{\Omega, \mathcal{F}\}$ with the filtration $\mathcal{F}_{n}$ on it, let $M$ be a regular set of measures. Suppose that the super-martingale $\left\{f_{m}, \mathcal{F}_{m}\right\}_{m=0}^{\infty}$ relative to the set of measures $M$ satisfy the conditions

$$
\left|f_{m}\right| \leq C \xi_{0}, m=\overline{1, \infty}, \xi_{0} \in A_{0}, 0<C<\infty,
$$

then the necessary and sufficient conditions for it to be a local regular one is belonging it to the set $K$.

Proof. The necessity is evident.

The sufficiency. Suppose that $\left\{f_{m}, \mathcal{F}_{m}\right\}_{m=0}^{\infty}$ is a local regular super-martingale. Then, there exists a nonnegative adapted random process $\left\{\bar{g}_{m}^{0}\right\}_{m=1}^{\infty}, E^{P} \bar{g}_{m}^{0}<\infty, m=\overline{1, \infty}$, and a martingale $\left\{M_{m}\right\}_{m=0}^{\infty}, E^{P}\left|M_{m}\right|<\infty, m=\overline{1, \infty}, P \in M$, such that

$$
f_{m}=M_{m}-\sum_{i=1}^{m} \bar{g}_{i}^{0}, m=\overline{0, \infty} .
$$

The inequalities $f_{m}+C \xi_{0} \geq 0, m=\overline{1, \infty}$, give the inequalities

$$
f_{m}+C E^{P}\left\{\xi_{0} \mid \mathcal{F}_{m}\right\} \geq 0, m=\overline{0, \infty} .
$$

From the inequalities (187), it follows that the super-martingale $\left\{f_{m}, \mathcal{F}_{m}\right\}_{m=0}^{\infty}$ is a uniformly integrable one relative to the regular set of measures $M$. The martingale $\left\{E^{P}\left\{\xi_{0} \mid \mathcal{F}_{m}\right\}, \mathcal{F}_{m}\right\}_{m=0}^{\infty}$ relative to the regular set of measures $M$ is also uniformly integrable one.

Then, $M_{m}+C E^{P}\left\{\xi_{0} \mid \mathcal{F}_{m}\right\} \geq 0, m=\overline{0, \infty}$. Since $0<E^{P}\left[M_{m}+C E^{P}\left\{\xi_{0} \mid \mathcal{F}_{m}\right\}\right]=f_{0}+C<\infty$, we have $E^{P} \sum_{i=1}^{m} \bar{g}_{i}^{0}<f_{0}+C$. Let us put $g_{\infty}=\lim _{m \rightarrow \infty} \sum_{i=1}^{m} \bar{g}_{i}^{0}$. Using the uniform integrability of $f_{m}$ and $\sum_{i=1}^{m} \bar{g}_{i}^{0}$, we can pass to the limit in the equality

$$
E^{P}\left(f_{m}+\sum_{i=1}^{m} \bar{g}_{i}^{0}\right)=f_{0}, P \in M,
$$

as $m \rightarrow \infty$. Passing to the limit in the last equality, as $m \rightarrow \infty$, we obtain

$$
E^{P}\left(f_{\infty}+g_{\infty}\right)=f_{0}, P \in M .
$$

Introduce into consideration a random value $\xi_{1}=\frac{f_{\infty}+C \xi_{0}+g_{\infty}}{f_{0}+C} \geq 0$. Then, $E^{P} \xi_{1}=1, P \in M$. From here, we obtain that $\xi_{1} \in A_{0}$ and for the super-martingale $f=\left\{f_{m}, \mathcal{F}_{m}\right\}_{m=0}^{\infty}$ the representation 


$$
f_{m}=f_{m}^{0} E^{P}\left\{\xi_{0} \mid \mathcal{F}_{m}\right\}+f_{m}^{1} E^{P}\left\{\xi_{1} \mid \mathcal{F}_{m}\right\}+f_{m}^{2} E^{P}\left\{\xi_{2} \mid \mathcal{F}_{m}\right\}, m=\overline{0, \infty}
$$

is valid, where $f_{m}^{0}=-C, f_{m}^{1}=f_{0}+C, f_{m}^{2}=-\sum_{i=1}^{m} \bar{g}_{i}^{0}, m=\overline{0, \infty}, \xi_{2}=1$. From the last representation, it follows that the super-martingale $f=\left\{f_{m}, \mathcal{F}_{m}\right\}_{m=0}^{\infty}$ belongs to the set $K$. Theorem 16 is proved.

Corollary 3. Let $f_{N}, N<\infty$, be a $\mathcal{F}_{N}$-measurable integrable random value, $\sup _{P \in M} E^{P}\left|f_{N}\right|<\infty$, and let there exist $\alpha_{0} \in R^{1}$ such that

$$
-\alpha_{0} M_{N}+f_{N} \leq 0, \omega \in \Omega,
$$

where $\left\{M_{m}, \mathcal{F}_{m}\right\}_{m=0}^{\infty}=\left\{E^{P}\left\{\xi \mid \mathcal{F}_{m}\right\}, \mathcal{F}_{m}\right\}_{m=0}^{\infty}, \xi \in A_{0}$. Then, a super-martingale $\left\{f_{m}^{0}+\bar{f}_{m}\right\}_{m=0}^{\infty}$ is a local regular one relative to the regular set of measures $M$, where

$$
\begin{gathered}
f_{m}^{0}=\alpha_{0} M_{m}, \\
\bar{f}_{m}= \begin{cases}0, & m<N, \\
f_{N}-\alpha_{0} M_{N}, & m \geq N .\end{cases}
\end{gathered}
$$

Proof. It is evident that $\bar{f}_{m-1}-\bar{f}_{m} \geq 0, m=\overline{0, \infty}$. Therefore, the super-martingale

$$
f_{m}^{0}+\bar{f}_{m}= \begin{cases}\alpha_{0} M_{m}, & m<N, \\ f_{N}, & m=N, \\ f_{N}-\alpha_{0} M_{N}+\alpha_{0} M_{m}, & m>N\end{cases}
$$

is a local regular one relative to the regular set of measures $M$. Corollary 3 is proved.

\section{Discrete Geometric Brownian Motion}

In this section, we construct for the discrete evolution of risk assets the set of equivalent martingale measures and give a new formula for the fair price of super-hedge. Let $\Omega_{1}^{0}=R^{1}, F_{1}^{0}=B\left(R^{1}\right)$, where $R^{1}$ is a real axis, $B\left(R^{1}\right)$ is a Borel $\sigma$-algebra of $R^{1}$. Let us put $\Omega_{i}=\Omega_{1}, F_{i}^{0}=F_{1}^{0}, i=\overline{1, \infty}$, and let us construct the infinite direct product of the measurable spaces $\left\{\Omega_{i}^{0}, \mathcal{F}_{i}^{0}\right\}, i=\overline{1, \infty}$. Denote $\Omega=\prod_{i=1}^{\infty} \Omega_{i}^{0}$. Under the $\sigma$-algebra $\mathcal{F}$ on $\Omega$, we understand the minimal $\sigma$-algebra generated by sets $\prod_{i=1}^{\infty} G_{i}, G_{i} \in \mathcal{F}_{i}^{0}$, where in the last product only the finite set of $G_{i}$ do not equal $\Omega_{i}^{0}$. On the measurable space $\{\Omega, \mathcal{F}\}$, under the filtration $\mathcal{F}_{n}$ we understand the minimal $\sigma$-algebra, generated by sets $\prod_{i=1}^{\infty} G_{i}, G_{i} \in F_{i}^{0}$, where $G_{i}=\Omega_{i}^{0}$ for $i>n$. Suppose that the points $t_{0}=0, t_{1}, t_{2}, \cdots, t_{n}, \cdots$, belongs to $R_{1}^{+}$with $\Delta t=t_{i}-t_{i-1}$ not depending on the index $i$. Let us consider the probability space $\{\Omega, \mathcal{F}, P\}$, where $P=\prod_{i=1}^{\infty} P_{i}^{0}, P_{i}^{0}=P_{1}^{0}, i=\overline{1, \infty}$, 


$$
P_{1}^{0}(A)=\frac{1}{[2 \pi \Delta t]^{1 / 2}} \int_{A} \mathrm{e}^{-\frac{y^{2}}{2 \Delta t}} \mathrm{~d} y, \quad A \in \mathcal{F}_{1}^{0} .
$$

Define on the set $t_{0}=0, t_{1}, t_{2}, \cdots, t_{n}, \cdots$, the discrete Brownian motion. We say that the random process $w\left(t_{i}\right), i=\overline{0, \infty}$, is a discrete Brownian motion, if on $\{\Omega, \mathcal{F}\}$ the joint distribution function is given by the formula

$$
\begin{aligned}
& P_{0}\left(w\left(t_{i_{1}}\right) \in A_{i_{1}}, \cdots, w\left(t_{i_{k}}\right) \in A\right)_{i_{k}} \\
& =\frac{1}{D} \int_{A_{\mathrm{i}_{1}} \times \cdots \times A_{i_{k}}} \mathrm{e}^{-\frac{y_{i_{1}}^{2}}{2 \Delta t_{i_{1}}}} \times \cdots \times \mathrm{e}^{-\frac{\left[y_{i_{k}}-y_{i_{k-1}}\right]^{2}}{2 \Delta t_{i_{k}}}} \mathrm{~d} y_{i_{1}} \cdots \mathrm{d} y_{i_{k}}, A_{i_{s}} \in \mathcal{F}_{i_{s}}^{0}, \\
& D=[2 \pi]^{k / 2}\left[\Delta t_{i_{1}} \times \cdots \times \Delta t_{i_{k}}\right]^{1 / 2}, \Delta t_{i_{s}}=t_{i_{s}}-t_{i_{s-1}} .
\end{aligned}
$$

So defined above the random process $w\left(t_{i}\right)$ on the set $t_{0}, t_{1}, t_{2}, \cdots, t_{n}, \cdots$, with $w(0)=0$, is a homogeneous one relative to the displacement on $k \Delta t$, where $k \geq 1$, and is a natural number, with the independent increments, the zero expectation and the correlation function $E^{P_{0}} w\left(t_{s}\right) w\left(t_{k}\right)=\min \left\{t_{s}, t_{k}\right\}$.

We assume that the evolution of non risk asset is given by the formula $B_{n}=\mathrm{e}^{r t_{n}}, n=\overline{0, \infty}$, where $r$ is an interest rate. Let us consider on $\{\Omega, \mathcal{F}, P\}$ the evolution of risk asset given by the law

$$
\bar{S}_{n}=S_{0} \mathrm{e}^{\left(\mu-\frac{\sigma^{2}}{2}\right) t_{n}+\sigma w\left(t_{n}\right)} .
$$

Further, we consider the discount evolution of the risk asset

$$
S_{n}=\frac{\bar{S}_{n}}{B_{n}}=S_{0} \mathrm{e}^{\left(\mu-\frac{\sigma^{2}}{2}-r\right) t_{n}+\sigma w\left(t_{n}\right)} .
$$

It is convenient to present these evolutions in the form

$$
S_{n}=\left(1+\rho_{n}\right) S_{n-1}, n=\overline{1, \infty},
$$

with $\rho_{n}=\mathrm{e}^{\left(\mu-\frac{\sigma^{2}}{2}-r\right) \Delta t+\sigma\left(w\left(t_{n}\right)-w\left(t_{n-1}\right)\right)}-1$.

On the probability space $\{\Omega, \mathcal{F}, P\}$ with the filtration $\mathcal{F}_{n}$ on it, for further investigations it is convenient to present the Brownian motion in the equivalent form. We present the Brownian motion by the sequence of random values $\zeta_{n}=\sum_{i=1}^{n} y_{i}, y_{i} \in \Omega_{i}^{0}, n=\overline{1, \infty}$, with the joint distribution functions

$$
\begin{gathered}
P\left(\zeta_{i_{1}} \in A_{i_{1}}, \cdots, \zeta_{i_{k}} \in A_{i_{k}}\right) \\
=\frac{1}{D} \int_{A_{i_{1}} \times \cdots \times A_{i_{k}}} \mathrm{e}^{-\frac{y_{i_{1}}^{2}}{22_{i_{1}} \Delta t}} \times \cdots \times \mathrm{e}^{-\frac{\left[y_{i_{k}}-y_{i_{k-1}}\right]^{2}}{2\left(i_{k}-i_{k-1}\right) \Delta t}} \mathrm{~d} y_{i_{1}} \cdots \mathrm{d} y_{i_{k}}, A_{i_{s}} \in \mathcal{F}_{i_{s}}^{0}, \\
D=[2 \pi]^{i_{k} / 2}[\Delta t]^{i_{k} / 2}\left[i_{1}\left(i_{2}-i_{1}\right) \times \cdots \times\left(i_{k}-i_{k-1}\right)\right]^{1 / 2} .
\end{gathered}
$$

Then, the discount evolution of the risk asset we can rewrite in the form

$$
S_{n}=S_{0} \mathrm{e}^{\left(\mu-\frac{\sigma^{2}}{2}-r\right) n \Delta t+\sigma \zeta_{n}}
$$


It is convenient to present discount evolution in the form

$$
S_{n}=\left(1+\rho_{n}\left(y_{n}\right)\right) S_{n-1}, n=\overline{1, \infty},
$$

with $\rho_{n}\left(y_{n}\right)=\mathrm{e}^{\left(\mu-\frac{\sigma^{2}}{2}-r\right) \Delta t+\sigma y_{n}}-1=\rho_{1}\left(y_{n}\right)$.

On the measurable space $\left\{\Omega^{N}, \mathcal{F}^{N}\right\}$ with the filtration $\mathcal{F}_{n}, n=\overline{1, N}$, on it, where $\Omega^{N}=\prod_{i=1}^{N} \Omega_{i}^{0}, \mathcal{F}^{N}=\prod_{i=1}^{N} \mathcal{F}_{i}^{0}$, we introduce into consideration the set of measures $M^{N}$. A measure $Q$ belongs to $M^{N}$, if $Q=\prod_{i=1}^{N} Q_{i}, Q_{i} \in M_{1}^{0}$. For every $\bar{Q} \in M_{1}^{0}$ the representation

$$
\begin{aligned}
& \bar{Q}(A)=\iint_{\Omega_{1}^{-} \Omega_{1}^{+}} \chi_{A}\left(y_{1}\right) \alpha\left(y_{1}, y_{2}\right) \frac{\rho_{1}^{+}\left(y_{2}\right)}{\rho_{1}^{-}\left(y_{1}\right)+\rho_{1}^{+}\left(y_{2}\right)} \mathrm{d} \mu\left(y_{1}, y_{2}\right) \\
& +\iint_{\Omega_{1}^{-} \Omega_{1}^{+}} \chi_{A}\left(y_{2}\right) \alpha\left(y_{1}, y_{2}\right) \frac{\rho_{1}^{-}\left(y_{1}\right)}{\rho_{1}^{-}\left(y_{1}\right)+\rho_{1}^{+}\left(y_{2}\right)} \mathrm{d} \mu\left(y_{1}, y_{2}\right), A \in \mathcal{F}_{1}^{0}, \\
& \Omega_{1}^{-}=\left\{y \in R^{1}, \rho_{1}(y) \leq 0\right\}=\left\{y \in R^{1}, y \leq-\frac{\left(\mu-\frac{\sigma^{2}}{2}-r\right) \Delta t}{\sigma}\right\} \text {, } \\
& \Omega_{1}^{+}=\left\{y \in R^{1}, \rho_{1}(y)>0\right\}=\left\{y \in R^{1}, y>-\frac{\left(\mu-\frac{\sigma^{2}}{2}-r\right) \Delta t}{\sigma}\right\},
\end{aligned}
$$

is valid, where $\rho_{1}(y)=\rho_{1}^{+}(y)-\rho_{1}^{-}(y), \quad \rho_{1}(y)=\mathrm{e}^{\left(\mu-\frac{\sigma^{2}}{2}-r\right) \Delta t+\sigma y}-1$, $\mu=P^{-} \times P^{+}$,

$$
\begin{aligned}
& P^{-}(A)=\frac{1}{[2 \pi \Delta t]^{1 / 2}} \int_{A} \mathrm{e}^{-\frac{y^{2}}{2 \Delta t}} \mathrm{~d} y, A \in B\left(\Omega_{1}^{-}\right), \\
& P^{+}(A)=\frac{1}{[2 \pi \Delta t]^{1 / 2}} \int_{A} \mathrm{e}^{-\frac{y^{2}}{2 \Delta t}} \mathrm{~d} y, A \in B\left(\Omega_{1}^{+}\right) .
\end{aligned}
$$

On the measurable space $\left\{\Omega_{1}^{-} \times \Omega_{1}^{+}, B\left(\Omega_{1}^{-}\right) \times B\left(\Omega_{1}^{+}\right)\right\}$, the random value $\alpha\left(y_{1}, y_{2}\right)$ satisfy the conditions

$$
\begin{gathered}
\mu\left(\left\{\left(y_{1}, y_{2}\right) \in \Omega_{1}^{-} \times \Omega_{1}^{+}, \alpha\left(y_{1}, y_{2}\right)>0\right\}\right)=P\left(\Omega_{1}^{+}\right) P\left(\Omega_{1}^{-}\right), \\
\iint_{\Omega_{1}^{-} \Omega_{1}^{+}} \alpha\left(y_{1}, y_{2}\right) \frac{\rho_{1}^{-}\left(y_{1}\right) \rho_{1}^{+}\left(y_{2}\right)}{\rho_{1}^{-}\left(y_{1}\right)+\rho_{1}^{+}\left(y_{2}\right)} \mathrm{d} \mu\left(y_{1}, y_{2}\right)<\infty, \\
\iint_{\Omega_{1}^{-} \Omega_{1}^{+}} \alpha\left(y_{1}, y_{2}\right) \mathrm{d} \mu\left(y_{1}, y_{2}\right)=1,
\end{gathered}
$$

for every bounded $\alpha\left(y_{1}, y_{2}\right)>0$, since $E^{P_{1}^{0}}\left|\rho_{1}(y)\right|<\infty$. Denote $M_{0}^{N}=M_{c}^{N}$ 
the convex linear span of the set of measures $M^{N}$. On the measurable space $\left\{\Omega^{N}, \mathcal{F}^{N}\right\}$ with the filtration $\mathcal{F}_{n}, n=\overline{0, N}$, on it, in correspondence with Theorem 8 , the set of measures $M_{0}^{N}$ is a regular set of measures with the random variable $\xi_{0}=\prod_{i=1}^{N}\left(1+\rho_{i}\left(y_{i}\right)\right)$, since the random value $\eta_{1}=\rho_{1}\left(y_{1}\right)$, figuring in Theorem 8 , is an integrable one relative to the measure $P_{1}^{0}$ and, therefore, $E^{Q} \xi_{0}=1, Q \in M_{0}^{N}$. It means that the set of equivalent martingale measures $M_{0}^{N}$ for the discount evolution $S_{n}=S_{0} \mathrm{e}^{\left(\mu-\frac{\sigma^{2}}{2}-r\right) n \Delta t+\sigma \zeta_{n}}$ of the risk asset contains more than one martingale measure. In this case, the financial market is an incomplete one.

Theorem 17. On the measurable space $\left\{\Omega^{N}, \mathcal{F}^{N}\right\}$ with the filtration $\mathcal{F}_{n}, n=\overline{0, N}$, on it, let the discount risk asset evolution is given by the formula $S_{n}=S_{0} \mathrm{e}^{\left(\mu-\frac{\sigma^{2}}{2}-r\right) n \Delta t+\sigma \zeta_{n}}$. For the payment function $f\left(S_{N}\right)$, satisfying the condition $\sup _{Q \in M_{0}^{N}} E^{Q} f\left(S_{N}\right)<\infty$, the fair price of super-hedge is giving by the formula

$$
\begin{aligned}
& \sup _{Q \in M_{0}^{N}} E^{Q} f\left(S_{N}\right) \\
& =\sup _{y_{i}^{1} \leq-d, y_{i}^{2}>-d, i=1, \bar{N}} \sum_{i_{1}=1, \cdots, i_{N}=1}^{2} f\left(S_{0} \prod_{s=1}^{N}\left(1+\rho\left(y_{s}^{i_{s}}\right)\right)\right) \prod_{s=1}^{N} \frac{\left|\mathrm{e}^{\sigma\left(d+y_{s}^{i_{s}+1}\right)}-1\right|}{\left|\mathrm{e}^{\sigma\left(d+y_{s}^{i_{s}+1}\right)}-\mathrm{e}^{\sigma\left(d+y_{s}^{i_{s}}\right)}\right|},
\end{aligned}
$$

where we put $d=\frac{\left(\mu-\frac{\sigma^{2}}{2}-r\right) \Delta t}{\sigma}, y_{s}^{3}=y_{s}^{1}$.

Proof. Since $R^{1}$ is a separable metric space then due to Lemma 7 the Borel $\sigma$-algebra $B\left(R^{1}\right)$ has the exhaustive decomposition. Therefore, the filtration $\mathcal{F}_{n}, n=\overline{0, N}$, has the exhaustive decomposition, due to Remark 1. Theorem 11 guarantee the formula for the fair price of super-hedge [5]. Due to Remark 3 after Theorem 8 , the set of measures $\prod_{i=1}^{n} \mu_{\left\{y_{i}^{1}, y_{i}^{2}\right\}}$, where

$$
\begin{gathered}
\mu_{\left\{y_{i}^{1}, y_{i}^{2}\right\}}(A)=\chi_{A}\left(y_{i}^{1}\right) \frac{\rho_{i}^{+}\left(y_{i}^{2}\right)}{\rho_{i}^{-}\left(y_{i}^{1}\right)+\rho_{i}^{+}\left(y_{i}^{2}\right)}+\chi_{A}\left(y_{i}^{2}\right) \frac{\rho_{i}^{-}\left(y_{i}^{1}\right)}{\rho_{i}^{-}\left(y_{i}^{1}\right)+\rho_{i}^{+}\left(y_{i}^{2}\right)}, \\
\left(y_{i}^{1}, y_{i}^{2}\right) \in \Omega_{i}^{-} \times \Omega_{i}^{+}, \Omega_{i}^{-}=\Omega_{1}^{-}, \Omega_{i}^{+}=\Omega_{1}^{+}, i=\overline{1, N},
\end{gathered}
$$

forms the extreme points of the convex set of measures $M_{0}^{N}$. The formula (208) is obtained by integration relative to the measure $\prod_{i=1}^{n} \mu_{\left\{y_{i}^{1}, y_{i}^{2}\right\}}$ of the random value $f\left(S_{N}\right)$ and taking the sup on the set of all extreme points. Theorem 17 is proved.

\section{Conclusions}

In the paper, we generalize the results of the paper [5]. Section 2 contains the de- 
finition of local regular super-martingales relative to the set of equivalent measures. Theorem 1 gives the necessary and sufficient conditions of the local regularity of a super-martingale. In spite of its simplicity, the Theorem 1 appeared very useful for the description of the local regular super-martingales.

Section 3 contains the important Definition 3 of the set of equivalent measures consistent with the filtration. In Lemma 3, we give an example of the set of equivalent measures consistent with the filtration. Theorem 2 contains the sufficient conditions under that there exists a nonnegative super-martingale on a measurable space with the set of measures consistent with the filtration. In Theorem 3, the sufficient conditions are founded which guarantee the existence on a measurable space a regular martingale.

Lemma 4 gives the sufficient conditions of the existence of a set of measures consistent with the filtration and such that the mean value of a nonnegative random value relative to all measures equals one.

Further the problem of constructing the set of equivalent measures satisfying the conditions of Lemma 4 is investigated. In Lemma 5 on a probability space we describe the set of equivalent measures satisfying the condition: the mean value of a random value relative to this set of measures equals zero. At last in this section, Theorem 4 gives the necessary and sufficient conditions of the local regularity of a nonnegative super-martingale relative to the equivalent set of measures.

In Section 4, in Lemma 6, we investigate the closure of the set of considered set of measure in the case of the countable space of elementary events. It is proved that in metrics (69) the closure of the set of considered set of measures contains the set of measures (70).

Further, we introduce the notion of the exhaustive decomposition of a measurable space. Lemma 7 states: if the measurable space is a complete separable metric space with the Borel $\sigma$-algebra on it then has an exhaustive decomposition.

Using this notion, in Lemma 8, we describe the closure of the considered set of measures relative to the pointwise convergence of measures and the closure of expectation values relative to this set of measures. Theorem 5 is a consequence of Lemma 5 and contains the description of the set of measures, being equivalent to the given measure, expectations relative to which equals one. Theorem 6 states the necessary and sufficient conditions when the set of measures (95) is consistent with filtration.

Theorem 7 states the necessary and sufficient conditions of the consistency with the filtration of the set of measure (95).

Using Lemma 5, in Lemma 9, we construct an example of the set of equivalent measures consistent with the filtration.

Further we consider an evolution of risk asset with memory. In Theorem 8, we describe completely the set of martingale measures for the considered evolution and prove that every martingale measure of this family is an integral over some measure on the set of extreme points of the set of martingale measures. Theorem 9 describes the closure of the mean value of integrable random values relative to 
the contractions of martingale measures onto the filtration. In Theorem 10 the bound for every nonnegative $\mathcal{F}_{n}$ measurable random value the mathematical expectation for which relative to every martingale measure is bounded by 1 is found. In Theorem 11, it is proved that every nonnegative super-martingale relative to the regular set of measures is a local regular one. The same statement, as in Theorem 11, it is proved in Theorem 12 in the case, as a super-martingale is bounded from below.

Section 5 contains the description of the local regular super-martingales. Using Theorem 1, we prove Theorem 14, giving the possibility to describe the local regular super-martingales. Further, we introduce a class $K$ of the local regular super-martingales relative to a regular set of measures. Theorem 15 states that every nonnegative uniformly integrable super-martingale relative to a regular set of measures belongs to the class $K$. The next Theorem 16 states that all super-martingales that are majorized by elements from the set $A_{0}$ also belong to the class $K$. At last, in corollary 3 , we give an example of the local regular super-martingale playing an important role in the definition of the fair price of the contingent claim [5].

Section 6 contains the application of the results obtained above to calculation of the fair price of super-hedge, when the risk asset evolves by the discrete geometric Brownian motion. In this case, we describe the set of regular measures. We find the set of extreme points of the regular set of measures. It is proved that the fair price of the super-hedge is given by the formula (208).

\section{Funding}

This work was supported in part by The National Academy of Sciences of Ukraine (Project No. 0118U003196).

\section{Conflicts of Interest}

The author declares no conflicts of interest regarding the publication of this paper.

\section{References}

[1] Kramkov, D.O. (1996) Optional Decomposition of Super-Martingales and Hedging in Incomplete Security Markets. Probability Theory and Related Fields, 105, 459-479. https://doi.org/10.1007/BF01191909

[2] Follmer, H. and Kramkov, D.O. (1997) Optional Decomposition Theorem under Constraints. Probability Theory and Related Fields, 109, 1-25. https://doi.org/10.1007/s004400050122

[3] Follmer, H. and Kabanov, Yu.M. (1996) Optional Decomposition Theorems in Discrete Time. Atti del convegno in onore di Oliviero Lessi, Padova, 25-26 Marzo 1996, 47-68.

[4] Follmer, H. and Kabanov, Yu.M. (1998) Optional Decomposition and Lagrange Multipliers. Finance and Stochastics, 2, 69-81. https://doi.org/10.1007/s007800050033 
[5] Gonchar, N.S. (2018) Martingales and Super-Martingales Relative to a Convex Set of Equivalent Measures. Advances in Pure Mathematics, 8, 428-462. https://doi.org/10.4236/apm.2018.84025

[6] El Karoui, N. and Quenez, M.C. (1995) Dynamic Programming and Pricing of Contingent Claims in an Incomplete Market. SIAM Journal on Control and Optimization, 33, 27-66. https://doi.org/10.1137/S0363012992232579

[7] Bouchard, B. and Nutz, M. (2015) Arbitrage and Duality in Nondominated Discrete-Time Models. The Annals of Applied Probability, 25, 823-859. https://doi.org/10.1214/14-AAP1011

[8] Gonchar, N.S. (2008) Mathematical Foundations of Information Economics. Bogolyubov Institute for Theoretical Physics, Kiev.

[9] Gonchar, N.S. (2015) Mathematical Model of Banking Operation. Cybernetics and System Analysis, 51, 378-399. https://doi.org/10.1007/s10559-015-9730-0

[10] Gonchar, N.S. and Terentieva, L.S. (2008) Default Risk Valuation of the Firm with the Special Process of Internal Yield. Journal of Automation and Information Sciences, 40, 57-71. https://doi.org/10.1615/JAutomatInfScien.v40.i8.60

[11] Gonchar, N.S. (2017) Banking and Risk Assessment. In: Jerzy, K., Ed., Banking. Services, Opportunity and Risks, Chapter 8, Nova Science Publisher, Inc., New York, 185-233.

[12] Kallianpur, G. (1980) Stochastic Filtering Theory. Springer, New York. https://doi.org/10.1007/978-1-4757-6592-2

[13] Chow, Y.S., Robbins, H. and Siegmund, D. (1971) Great Expectations: The Theory of Optimal Stopping. Houghton Mifflin Company, Boston.

[14] Gonchar, N.S. (2001) Stock Market and Economic Growth. Oberehy, Kiev. (In Ukrainian)

[15] Delbaen, F. and Schachermaer, W. (1994) A General Version of Fundamental Theorem of Asset Pricing. Mathematische Annalen, 300, 463-520. https://doi.org/10.1007/BF01450498

[16] Delbaen, F. and Schachermayer, W. (2006) The Mathematics and Arbitrage. Springer, Berlin.

[17] Dalang, R.C., Morton, A. and Willinger, W. (1990) Equivalent Martingale Measures and No-Arbitrage in Stochastic Securities Market Model. Stochastics and Stochastic Reports, 29, 185-201. https://doi.org/10.1080/17442509008833613

[18] Kreps, D.M. (1981) Arbitrage and Equilibrium in Economics with Infinitely Many Commodities. Journal of Mathematical Economics, 8, 15-35.

[19] Harrison, J.M. and Kreps, D.M. (1979) Martingales and Arbitrage in Multiperiod Securities Markets. Journal of Economic Theory, 20, 381-408. https://doi.org/10.1016/0022-0531(79)90043-7

[20] Harrison, J.M. and Pliska, S.R. (1981) Martingales and Stochastic Integrals in the Theory of Continous Trading. Stochastic Processes and Their Applications, 11, 215-260. 